\title{
Particle Physics and Cosmology
}

Lecture notes from the $100^{\text {th }}$ Les Houches Summer School on Post-Planck Cosmology, July $8^{\text {th }}-$ Aug $2^{\text {nd }} 2013$

\section{P. Pralavorio}

${ }^{1}$ CPPM, Univ. Aix-Marseille and CNRS/IN2P3, 163 avenue de Luminy, case 902, 13288 Marseille cedex 09 (France)

\begin{abstract}
Today, both particle physics and cosmology are described by few parameter Standard Models, i.e. it is possible to deduce consequence of particle physics in cosmology and vice versa. The former is examined in this lecture, in light of the recent systematic exploration of the electroweak scale by the LHC experiments. The two main results of the first phase of the LHC, the discovery of a Higgs-like particle and the absence so far of new particles predicted by "natural" theories beyond the Standard Model (supersymmetry, extra-dimension and composite Higgs) are put in a historical context to enlighten their importance and then presented extensively. To be complete, a short review from the neutrino physics, which can not be probed at LHC, is also given. The ability of all these results to resolve the 3 fundamental questions of cosmology about the nature of dark energy and dark matter as well as the origin of matter-antimatter asymmetry is discussed in each case.
\end{abstract}

Keywords SuperSymmetry · Extra Dimension . Cosmology $\cdot$ neutrino $\cdot$ Particle Physics $\cdot$ ATLAS · CMS $\cdot$ LHC

\section{Contents}

1 Introduction . . . . . . . . . . . . . . 1

2 Standard Model of Particle Physics and Cosmology . 2

3 Beyond Standard Model and Cosmology . . . . . . . 14

4 Conclusions ....................... 28

\section{Introduction}

The description of particle physics interactions and gravity in a common framework is still an ongoing effort but it was mostly speculative or purely philosophical at the

${ }^{\mathrm{a}} \mathrm{e}-\mathrm{mail}$ : pascal.pralavorio@cern.ch beginning of the $\mathrm{XX}^{\text {th }}$ century. The first attempt in this direction was to introduce a cosmological constant $\Lambda$ in the theory of gravitation. However the discovery of the universe expansion in the 20's forced Einstein to abandon this constant. In the 30 's, pioneering works from Lemaitre on primeval atom and identification between $\Lambda$ and the vacuum energy were poorly received by the community, and particle physics developed apart from cosmology for more than 30 years. The subject reappeared in a seminal paper entitled "The Universe as a Hot Laboratory for the Nuclear and Particle Physicist" by Zel'dovich in the late 1960s. At the beginning of the 1980s, the construction of Grand Unified Theories (GUT) at very high energy scale $\mathrm{O}\left(10^{15-16}\right) \mathrm{GeV}$ connected finally particle physics and cosmology. In simple words, "(...) the universe is the only machine we have that can test these GUT ideas. It's the world's biggest particle accelerator (...). But it's hard to use because all the experiments happened only once, (...) a long time ago" [1. The connection between particle physics and cosmology is nicely illustrated by the cosmic serpent shown in Fig. 1.

Today, benefiting from the huge technological developments during the last century, gigantic underground accelerators and spacecrafts probe everyday these two extreme realizations of physics. So what is more precisely the situation of particle physics and cosmology? At the end of the last century both fields were mature enough to give birth to "Standard Models". Both only need few free parameters to explain the huge wealth of data even if most of these parameters could not be explained yet in terms of a fundamental theory. But what is new is that these models enable to deduce consequence of particle physics in cosmology and vice verse, one of the most outstanding result of modern science. As confirmed by the latest Planck results, cosmology 


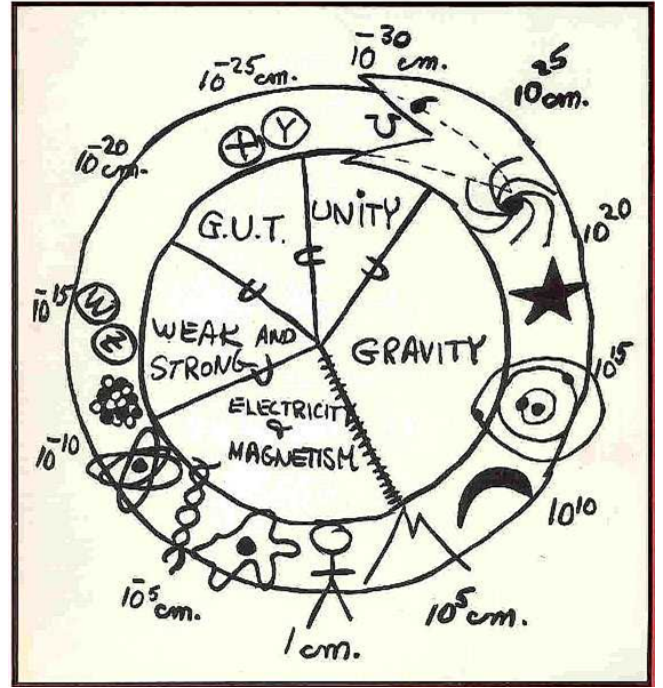

Fig. 1 S. Glashow serpent swallowing its tail, illustrating the interconnection between particle physics and cosmology [1.

provides three fundamental questions on today's universe:

1. What is dark energy?

2. What is dark matter?

3. Why is there matter and not antimatter (baryogenesis)?

May be part (or all) of these questions can be resolved by particle physics. Section 2 discusses, in light of the recent collider results, how particle physics could contribute to the solution of the first question. Section 3 presents the status of the collider and neutrino experiment searches for new particles beyond the Standard Model that could be able to solve the two last questions. Section 4 concludes by giving a tentative answer to these three questions using particle physics.

Particle physics results discussed in this lecture are a digest of the Large Hadron Collider (LHC) experiment publications by July 2013. At LHC, proton-proton collisions were recorded between spring 2010 and autumn 2012. More than 500 'LHC' papers were produced by July 2013 (comparable to the production of the last 10 years in Particle physics before LHC start). The quantity of data recorded by general purpose experiments ATLAS and CMS are proportional to the integrated luminosity $L$, expressed in $\mathrm{fb}^{-1}$, as the number $N$ of events expected for a given process of crosssection $\sigma$ is $N=L \sigma$. Cross-sections are expressed in pb $\left(10^{-36} \mathrm{~cm}^{2}\right)$ or fb $\left(10^{-39} \mathrm{~cm}^{2}\right)$. LHC accumulated $5 \mathrm{fb}^{-1}$ and $20 \mathrm{fb}^{-1}$ of data at a center of mass energy $\sqrt{s}=7 \mathrm{TeV}$ and $8 \mathrm{TeV}$, respectively. This two data sets are referred to as 'LHC Run I'. In this lecture, energy is expressed in $\mathrm{MeV}\left(10^{-3} \mathrm{GeV}\right), \mathrm{GeV}$ or
$\mathrm{TeV}\left(10^{3} \mathrm{GeV}\right)$. It corresponds to temperature and to the inverse of a distance $\left(\mathrm{m}^{-1}\right)$ and time $\left(\mathrm{s}^{-1}\right)$ when using natural units $\hbar=c=k_{B}=1$. Useful physical or astrophysical constants are the Planck mass, $M_{P l}=\sqrt{\hbar c / G_{N}} \simeq 1.2 \times 10^{19} \mathrm{GeV}$, where $G_{N}$ is the Newtonian gravitational constant, the Fermi coupling constant, $G_{F} /(\hbar c)^{3} \simeq 1.2 \times 10^{-5} \mathrm{GeV}^{-2}$, which characterizes the weak interaction and the fine-structure constant, $\alpha=e^{2} /\left(4 \pi \varepsilon_{0} \hbar c\right) \simeq 7.3 \times 10^{-3}$, which characterizes the electromagnetic (EM) interaction.

\section{Standard Model of Particle Physics and Cosmology}

Our current best guess of the early universe and its connection with particle physics is presented in Fig. 2. It is very interesting to note that only the last epochs of the early universe, corresponding to $10^{-2}<t(\mathrm{~s})<10^{13}$ $\left(10^{-10}<T_{R}(\mathrm{GeV})<10^{-2}\right)$, have been probed experimentally by Big Bang nucleosynthesis (BBN) and cosmic microwave background (CMB). This tells us that the post-inflationary reheating temperature $T_{R}$ of the universe have reached at least $10 \mathrm{MeV}$ [2. It is of course possible to access indirectly hotter epochs, but their direct exploration will require the very challenging detection of the cosmic neutrino background ( $\mathrm{CvB})$ and/or gravitational waves.

With the advent of particle physics colliders at the end of the 50's, it is however possible to produce experimentally energies much higher than $10 \mathrm{MeV}$. The most powerful one today is the LHC with $\sqrt{s}=8 \mathrm{TeV}$, 6 order of magnitude beyond what BBN can probe. Therefore developments in particle physics will directly benefit to cosmology - even if it is complicated by the irrelevance of gravity in particle physics experiments. It goes without saying that much higher energy collisions happen everyday in the universe (even on earth via cosmic ray interaction with the atmosphere), but particle physics colliders provide a unique setting for systematics studies.

After a brief historical review of particle physics and earlier connections with cosmology in Section 2.1 the basics of the Standard Model (SM) of Particle Physics as well as LHC collider and experiments are reminded in Section 2.2 and 2.3 respectively. An overview of the Higgs-like particle discovery and its consequences for the early universe follows in Section 2.4. Finally an explanation of the current SM limitations and its possible extensions are given in Section 2.5 


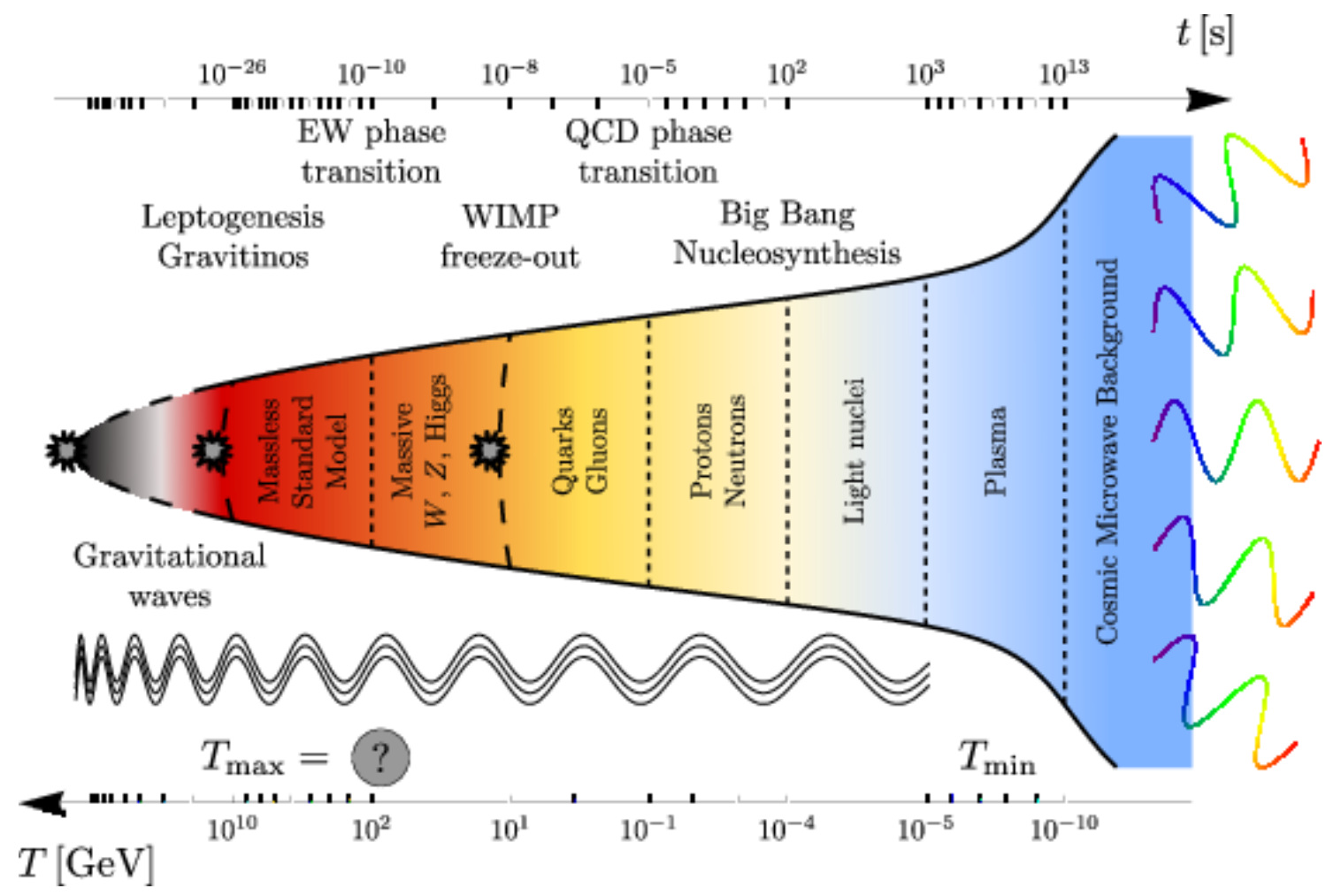

Fig. 2 Chronology of the hot thermal phase of the early universe. The top scale shows the time after the Big Bang and the bottom scale the corresponding post-inflationary reheating temperature [3].

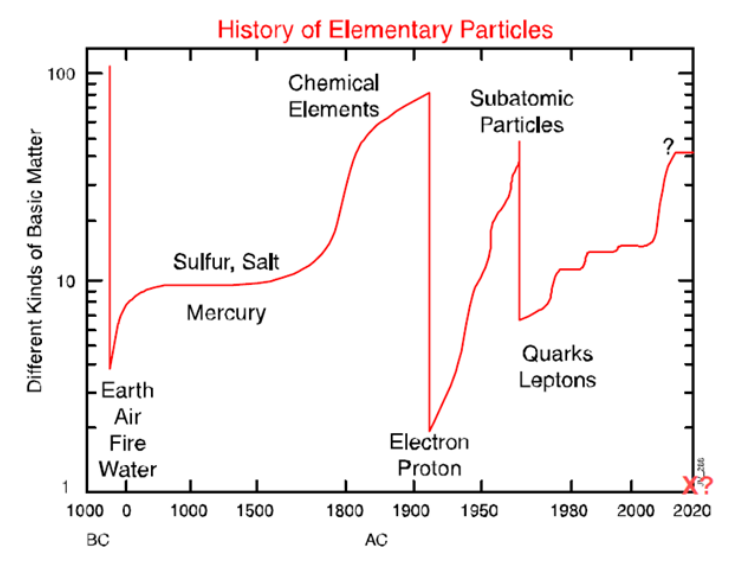

Fig. 3 Variation of the number of elementary particles as a function of time.

\subsection{History of Particle Physics and Cosmology}

Figure 3 shows the evolution of the number of elementary particles as a function of time. Three dramatic changes can be seen: the first one begins at the time of the ancient Greeks, who classified basic matter in four categories (Earth, Air, Fire and Water) and culminates when Mendeleev invented the periodic table of elements. A second era opens at the beginning of $\mathrm{XX}^{\text {th }}$ century when Niels Bohr described the hydrogen atom with two recently discovered particles: the proton 1 and the electron [4,5]. He computed the radius of the atom as:

$a_{0}=\frac{4 \pi \varepsilon_{0} \hbar^{2}}{m_{\mathrm{e}} e^{2}}=\frac{\hbar}{m_{\mathrm{e}} c \alpha} \sim \mathrm{O}\left(10^{-10}\right) \mathrm{m}$

At this atomic scale, lots of new phenomena were discovered and a new description of microscopic nature, quantum mechanics, emerges in the 20's. Later, thanks to the development of detection technology, lots of new particles were discovered first by studying cosmic rays and then as decay products of colliding particles. Late in the 60 's it was finally possible to make sense of all these new particles with quantum field and gauge theories. More specifically, the unification of electromagnetism and weak interaction 6, 6,7,8 including the Higgs mechanism 9, 10, 11, 12, 13, 14 allowed a coherent description of leptons [15. Similar evolution took place in the hadronic sector with the quark model [16] and the description of the strong force [17, 18, 19]. Hence the third era of elementary particles. Spectacularly all particles discovered until then could be explained in terms of these new elementary particles. The Model

${ }^{1}$ In fact the atomic nucleus at the time N. Bohr wrote his paper. 
was completed by the prediction of 3 copies of a 2 -fold fermion family [20] and the discovery of weak neutral currents [21,22, a direct consequence of the EM and weak unification. J. Ilioupolos announced officially the birth of the Standard Model, later called SM, with 19 parameters at the ICHEP conference in London in July 1974. Since then, the SM has been beautifully confirmed by all experiments. All new particles discovered fit perfectly in the model framework 2 , the leptons tau 23. and neutrino-tau [24, the quarks charm [25, 26], bottom [27] and top [28,29], as well as the gluon [30,31, $32,33], W$ [34,35], $Z$ [36,37] and recently the Higgs boson 38,39 .

Cosmology has followed similar historical evolution with abrupt changes: the Geocentric model (Aristotle) was turned into the Heliocentric model with elliptic orbits (Copernicus, Kepler). Later, the static uniform universe of Newton, Descartes and Kant, based on the classical gravity was turned into a static uniform universe based on General Relativity by Einstein in 1916. These static models were superseded by the dynamical hot Big Bang Model of Friedmann-Lemaitre in the 20's, complemented by the theory of inflation in the early 80 's which solved the horizon, flatness and magneticmonopole problems. Since 1998 and the concordant results obtained by SuperNovae, CMB and BAO experiments, it is considered as the Standard Model of Cosmology $(\Lambda \mathrm{CDM})$ with 6 free parameters.

There are numerous examples of cross-feeding between cosmology and particle physics. The most striking one dates back to 1900 when Max Planck postulated the quantization of the energy $(E=h \nu)$ to describe correctly the black body radiation. For particle physics, this was a first seed in the quantum mechanics garden that will flourish during the $\mathrm{XX}^{\text {th }}$ century. For cosmology, this provided a very strong argument in favor of the Big Bang theory in the 70's when the CMB temperature spectrum followed exactly the Planck's black body description with $T \sim 2.7 \mathrm{~K}$. More recently nucleosynthesis and the constraints on the mass and number of neutrinos are also good examples of this fruitful connection.

\subsection{Standard Model of Particle Physics}

The main features of the current version of the SM of Particle Physics are now given. A more thorough review can be found for example in [40. The Standard Model is a non abelian gauge field theory based on the symmetry group $S U(3)_{C} \otimes S U(2)_{L} \otimes U(1)_{Y} . S U(3)_{C}$ denotes the

${ }^{2}$ It is noticeable that all fermion discoveries were made in the US whereas the boson discoveries were made in Europe ! color $(C)$ group of Quantum Chromo Dynamics (QCD). $S U(2)_{L} \otimes U(1)_{Y}$ describes the electroweak (EW) interactions where the weak hypercharge $Y$ is the $\mathrm{U}(1)$ generator and can be linked to the electric charge $(Q)$ and the Weak Isospin $\left(T_{3}\right)$ by the formula $Y=2\left(Q-T_{3}\right)$. In total, the SM counts 58 objects, 118 degrees of freedom and 28 free parameters, that will be detailed in this section.

\subsubsection{SM Fields}

The Standard Model includes 45 massive fermion fields arranged in left-handed SU(2) doublets and right-handed $\mathrm{SU}(2)$ singlets as shown in Fig. 4. Since parity is maximally violated by the weak interaction 41, there is no right-handed neutrinos and only left-handed fermions (and right handed anti-fermions) are sensitive to the weak interaction. The primes on down-type quarks and neutrinos correspond to gauge eigenstates. They are linked to the mass eigenstates by two $3 \times 3$ mixing matrices called Cabibbo-Kobayashi-Maskawa (CKM) and Pontecorvo-Maki-Nakagawa-Sakata (PMNS) respectively. There are also 12 gauge boson fields corresponding to the different force carriers of the interactions. Nine are massless, the photon and the 8 colored gluons for electromagnetic and strong interactions respectively. Because it is a short-range force, the gauge bosons of the weak interaction need to be very massive, of the order of the weak scale. However this is not possible since $S U(2)_{L} \otimes U(1)_{Y}$ is conserved. A solution is provided by the Higgs mechanism. A complex scalar SU(2) doublet $\phi \equiv\left(\begin{array}{c}\phi^{+} \\ \phi^{0}\end{array}\right)$ is introduced with a tree-level potential:

$V\left(\phi^{\dagger} \phi\right)=-\frac{m_{H}^{2}}{2} \phi^{\dagger} \phi+\lambda\left(\phi^{\dagger} \phi\right)^{2}$

With $-m_{H}^{2}<0$ and $\lambda>0, V$ has a "Mexican hat" shape with an infinity of non trivial vacua. The vacuum expectation value (vev) of $\phi$ can be expressed as:

$v=\sqrt{m_{H}^{2} /(2 \lambda)}=\left(\sqrt{2} G_{F}\right)^{-1 / 2} \simeq 246 \mathrm{GeV}$

and $\phi$ can be developped as $\left(\begin{array}{c}H^{+} \\ \frac{1}{\sqrt{2}}\left(v+\operatorname{Re}\left(H^{0}\right)+i \operatorname{Im}\left(H^{0}\right)\right.\end{array}\right)$.

The Higgs potential is completely determined once $m_{H}$ is known. By choosing a particular ground state, the gauge symmetry $S U(2)_{L} \otimes U(1)_{Y}$ gets spontaneously broken in $\mathrm{U}(1)_{Q}$. The massless Goldstone bosons $\left(H^{+}\right.$, $H^{-}$and $\left.\operatorname{Im}\left(H^{0}\right)\right)$ generated by this symmetry breaking mix with the massless gauge bosons $W^{1,2,3}$, as illustrated in Fig. 5. Because of the Higgs vev, the Higgs mechanism generates three massive bosons $\left(W^{+}, W^{-}\right.$, $Z^{0}$ ) for the weak interaction, one massless boson for the 
EM interaction $(\gamma)$ and one massive scalar particle $(H$, the Higgs boson).

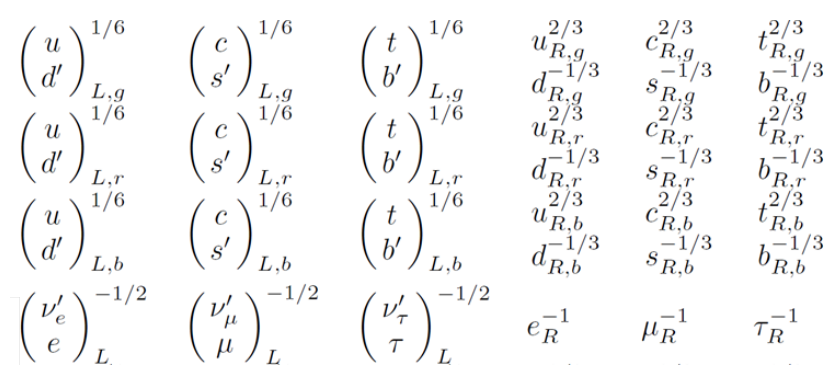

Fig. 4 SM fermion fields with their associated charges ( $Y$ top right, $C=g, r, b$ bottom right of each doublet or singlet) and chirality (bottom right of each doublet or singlet) 42 .

$$
\begin{array}{ccc}
W^{1}, W^{2}, H^{+}, H^{-} & \longrightarrow & W^{+}, W^{-} \\
W^{3}, B, \operatorname{Im}\left(H^{0}\right) & \longrightarrow & \gamma, Z \\
g \times 8 & & \\
\operatorname{Re} H^{0} & \longrightarrow & H
\end{array}
$$

Fig. 5 SM boson fields before and after EW symmetry breaking [42].

\subsubsection{SM Parameters}

Let's now enumerate the free parameters of the model. The interaction between scalar and Dirac fields is described by Yukawa-type Lagrangian $\mathcal{L}=-\lambda_{Y} \bar{\psi} \phi \psi$. It can be rewritten as $-m / v\left(\bar{\psi}_{R} \psi_{L}+\bar{\psi}_{L} \psi_{R}\right)^{*}$, where $\lambda_{Y}$ is a real coupling constant, $m$ is the Dirac mass and $\psi_{L}$ $\left(\psi_{R}\right)$ are the left-handed (right-handed) spinors. In the fermion sector, Yukawa-type couplings give 12 free parameters corresponding to the fermions masses. CKM and PMNS matrices can be parametrized by 3 angles and $1 \mathrm{CP}$ violating phase each [43. Assuming that the neutrino is of Majorana type, 2 other parameters are necessary for the PMNS matrix, giving a total of 10 parameters for the fermion mixing matrices. In the boson sector, the electroweak part needs 4 parameters $\left(\alpha, m_{Z}\right.$, $v, m_{H}$ for example) and the strong sector 2 parameters $\left(\alpha_{S}\right.$ coupling constant and a strong $\mathrm{CP}$ phase, $\Theta_{\mathrm{QCD}}$, very small but not null).

Overall most of the 28 fundamental SM parameters ${ }^{3}$ are a consequence of the presence of the Higgs

\footnotetext{
${ }^{3}$ Originally, the neutrinos were assumed massless and the PMNS matrix diagonal, hence the 19 parameters mentioned in Section 2.1.
}

field. Before 2012, all these parameters have been measured by experiments, except $7\left(m_{H}, \Theta_{\mathrm{QCD}}\right.$, the two Majorana phases of the PMNS matrix and the 3 neutrino masses) which are only constrained. However these constraints are generally weak. Therefore final values could have dramatic consequences on cosmology: $m_{H}$ will be discussed extensively in the following, while $\Theta_{\mathrm{QCD}}$ and neutrino parameters are discussed in Section 3.4 and 3.5 respectively.

\subsubsection{The Electroweak fit}

The Higgs boson occupies a peculiar place in the Standard Model and its discovery (or proof of its nonexistence) was, after the discovery of the intermediate vector bosons of the weak interaction in 1983, a major milestone of experimental particle physics. In 1974, $m_{H}$ could span from the electron mass to $M_{P l}$. A way to narrow down the mass region, and therefore help the experiments, is to use the presence of the Higgs particle in quantum corrections computed by perturbation theory, also called 'radiative corrections'. These corrections come from virtual particles constantly produced out of nothing that violate the energy-conservation law by borrowing an amount of energy $E$ from the vacuum for a very short time of at most $\hbar / E$. In the electroweak sector these corrections are generally small compared to tree-level but still sizable. Therefore very precisely measured observables at the $Z$-pole and from $W$ mass measurements allow indirect constraints on undiscovered particle masses as demonstrated for the top mass in Fig. 6. Before the LHC start, the EW fit favored a Higgs mass around $100 \mathrm{GeV}$ as shown in Fig. 7 .

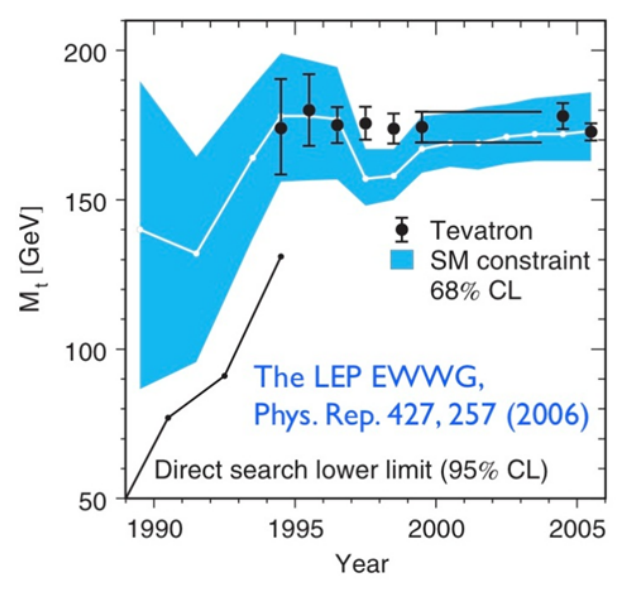

Fig. 6 Predictions from the EW Fit compared to experimental results for top mass 44. 


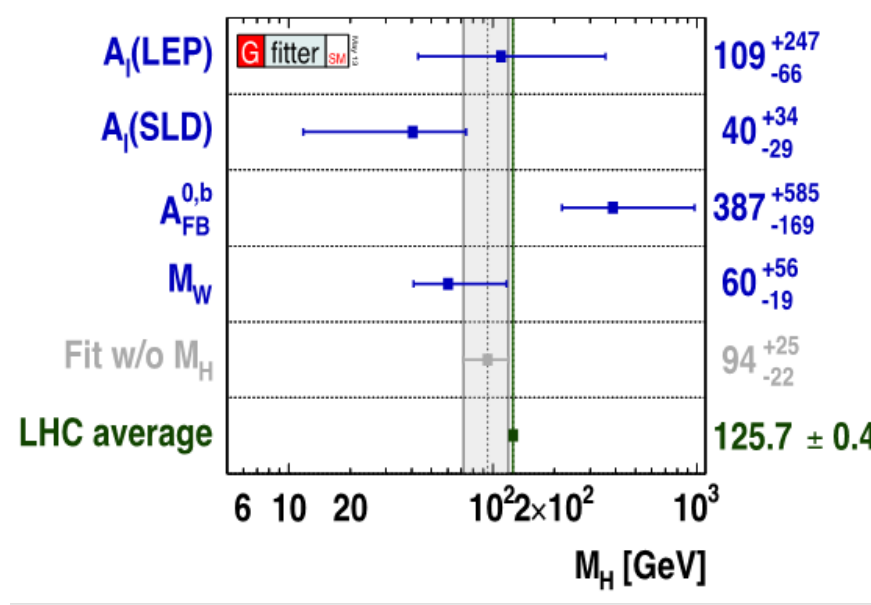

Fig. 7 Predictions from the EW Fit compared to experimental results for Higgs mass [45].

\subsection{LHC: collider, experiments and physics goals}

Following the Heisenberg uncertainty principle, Ex > $\hbar c$, probing lower distance $x$ requires higher available energy $E$. For example to probe $x=\mathrm{O}\left(10^{-15}\right)$ m, i.e. the proton radius, requires an energy of $0.1 \mathrm{GeV}$ and therefore a moderate size apparatus. A nice illustration is provided by the charged pion of mass $0.1 \mathrm{GeV}$, responsible for the strong nuclear force and close to the QCD scale $\Lambda_{\mathrm{QCD}} \sim 0.2 \mathrm{GeV}$ : it was discovered in photographic plates by looking at atmospheric cosmic rays [46] and then produced by the $5 \mathrm{~m}$ diameter UCBerkeley's cyclotron [47.

The next interesting scale to probe is the Fermi or Weak scale $\left(\Lambda_{\mathrm{EW}}\right)$ defined as:

$\Lambda_{\mathrm{EW}}=G_{F}{ }^{-1 / 2} \sim \mathrm{O}\left(10^{2}\right) \mathrm{GeV}$

which corresponds to typical size of $\mathrm{O}\left(10^{-18}\right) \mathrm{m}$. Applying a linear scaling to guess the accelerator size gives a ring of $\mathrm{O}(10) \mathrm{km}$. Giant size accelerator were therefore constructed to gradually reach this scale (see Fig. 8). The first one to succeed was the proton-antiproton Sp $\bar{p} S$ collider at CERN in the 1980's enabling the discovery of the $W$ and $Z$ bosons. Later $\mathrm{e}^{+}-\mathrm{e}^{-}$LEP and SLC colliders and the proton-antiproton Tevatron collider at FermiLab studied these new particles in great details as discussed in Section 2.2.3. However none were able to discover the Higgs boson because of limited statistics (Tevatron) or too low center-of-mass energy (SLC, LEP).

To cure both of these problems, it was decided in 1984 to construct a hadron collider, able to reach several times the center of mass energy of the Tevatron by using superconducting magnets, the only technology able to bend several $\mathrm{TeV}$ proton beam in the 27 - $\mathrm{km}$ LEP tunnel. To increase the luminosity by orders of magnitude, the collision of two proton beams (instead of proton-antiproton) is necessary and possible with 2-in-1 cryodipoles 48. The increase of the number of protons per bunch to $10^{11}$ causes however an increase of the number of "pile-up" interactions per crossing, see Fig. 11, that reached up to 35. This challenge, anticipated by the LHC experiments, leads to the construction of highly granular and radiation-hard detectors. The net result is that the LHC accumulated $L$ $\sim 10 \mathrm{fb}^{-1}$ by mid-2012, two years after starting collisions at $\sqrt{s}=7-8 \mathrm{TeV}$, see Fig. 10 . This corresponds to what the Tevatron recorded during 24 years. Thanks to these improvements in $\sqrt{s}$ and $L$, the cross-sections of interesting processes (bottom right part of Fig. 9) are greatly enhanced. Therefore on top of being a Higgs factory, LHC offers exciting possibilities to cover the region 0.1-O(1) $\mathrm{TeV}$ where physics beyond Standard model could be hidden, see Section 3 .

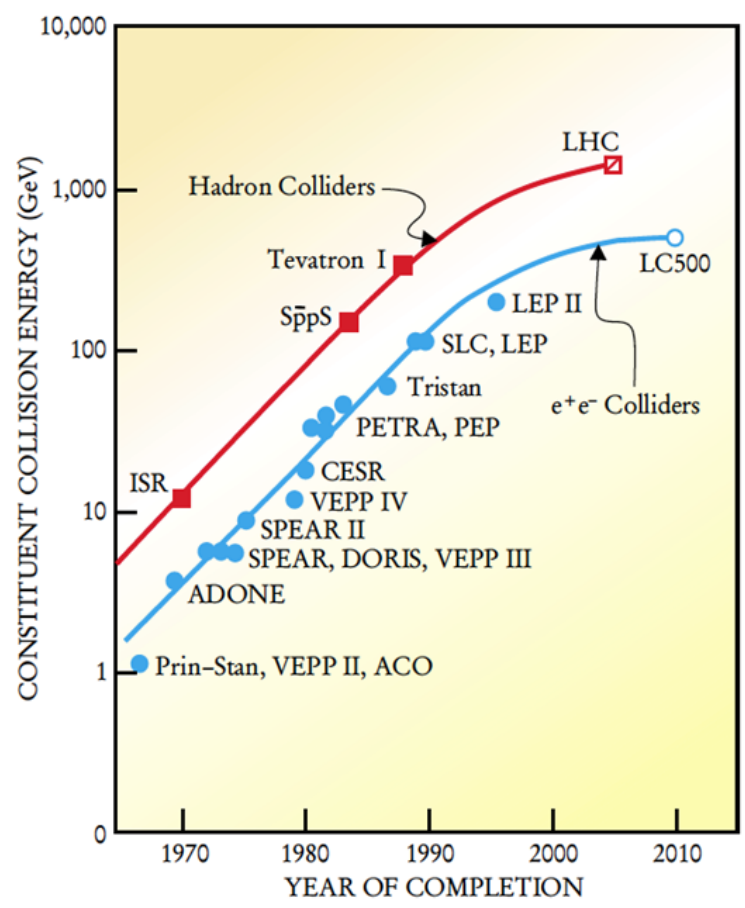

Fig. 8 Variation of the constituent energy as a function of completion time for different colliders. Constituent energy is $\sqrt{s} / 6$ for hadronic colliders, to account for proton compositeness, and LHC refers to LHC runII [49].

Four detectors have been built at the collision points located on the $100 \mathrm{~m}$ underground LEP Tunnel. Among the four, two general-purpose experiments [50] and 51] were designed to understand the origin of the EW symmetry breaking mechanism, Higgs or something else, and be sensitive to any sign of new physics around the 


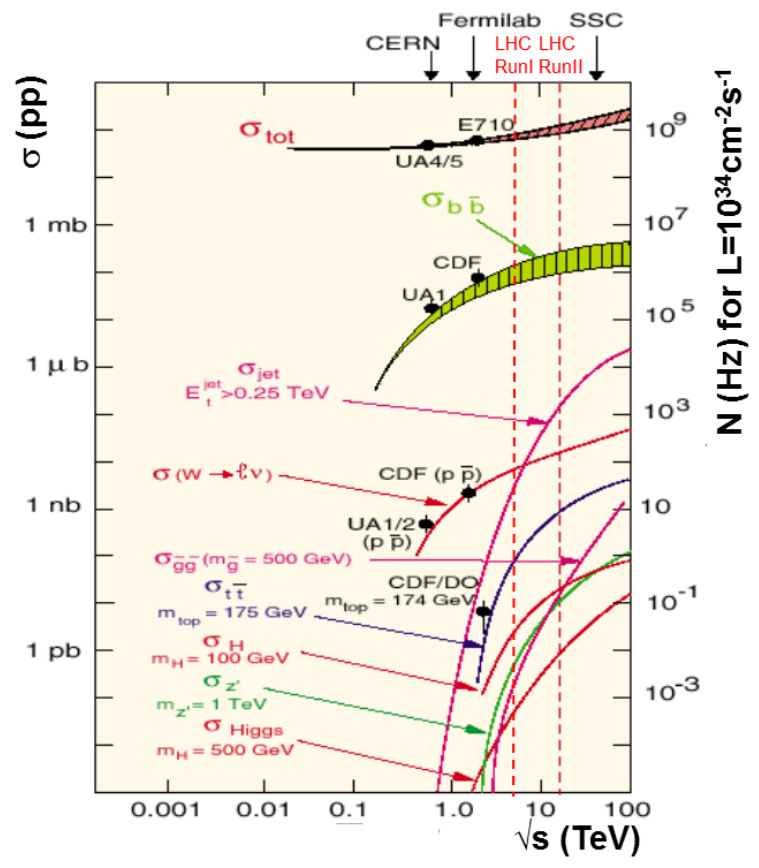

Fig. 9 Cross-section for various physics processes at different hadron colliders.

EW scale. Because of the huge complexity of a detector ready to cope with proton-proton collision every $25 \mathrm{~ns}$ and high pile-up conditions, world wide collaborations of few thousands of physicists and engineers were setup, giving to these projects a flavor of modern cathedral dedicated to science. Note that even if this is one of the most complex and ambitious project ever built, it is comparable in cost to other large-size projects [52]. The two detectors were based on two different technologies for the central magnets used to bend the charged particle trajectories: CMS uses a 4-Tesla superconducting solenoid magnet of $3 \mathrm{~m}$ radius while ATLAS choose a smaller central solenoid ( $2 \mathrm{~T}$ and $1.2 \mathrm{~m}$ radius) complemented by outer toroids [53]. These choices influence all the other detector technologies and especially the electromagnetic calorimeters, key to measure the kinematics of electrons and photons. CMS chose 75000 scintillating $\mathrm{PbWO}_{4}$ crystals with an excellent energy resolution but extremely low light yield while ATLAS built a granular (200k channels) lead/liquid argon sampling calorimeter, a robust and well known technology, with poorer energy resolution at low energy but comparable to CMS in the $0.1-1 \mathrm{TeV}$ energy range. This complementarity was a key element of the Higgs discovery (Section 2.4.1). Another important part of the success was the ability of ATLAS and CMS to use more than $90 \%$ of the high quality data produced by LHC for physics analysis, demonstrating the excellent functioning of both experiments.

Before entering the discovery phase, the rapidly growing luminosity allowed to check in details all known SM processes and especially those related to the most massive particles ( $W, Z$ and top), generally the major source of background for searches. Building on previous experiments and latest developments in theory computation beyond leading orders, an excellent agreement was found between predictions and data.

\subsection{Higgs and Cosmology}

As explained before, the Higgs is considered as the cornerstone of the SM of Particle Physics. Its discovery and its properties extracted from the first LHC run will be first discussed and the implication of these new results for cosmology will be presented at the end of the section.

\subsubsection{The Higgs discovery}

At LHC, the Higgs production is completely dominated by the gluon fusion process which occurs via one-loop 'triangle' heavy fermion (mainly top), since the Higgs can not directly couples to massless gluon. Even if this process is in principle suppressed $\mathrm{O}\left(\alpha_{S}^{2}\right)$, it benefits from the gluon domination at low momentum fraction in the proton and thus from higher $\sqrt{s}$. More precisely, the gluon fusion cross-section is multiplied by $\sim 25$ between Tevatron and LHC run I. Sub-dominant production processes are Vector Boson Fusion (VBF) and $t \bar{t} H$ fusion, where 2 additional quarks (light and top quark respectively) are produced with the Higgs. Finally vector boson Higgstrahlung $(W H, Z H)$ is also possible. Since beyond leading order processes over dominate the production, a huge theoretical effort was engaged to compute accurately the total cross-section for each mass of the Higgs boson in the range $80-1000 \mathrm{GeV}$ [54], see Fig. 12. Similarly, efforts went into determining the Higgs decay branching ratios [55], as summarized in Fig. 13 . Before the start of the LHC, the theoretical uncertainties on main production and decay modes were typically $5-10 \%$ or below.

As already mentioned, $m_{H}$ was not constrained by the theory when it was introduced in the SM. The first effort to compute its decay branching ratios dates back from 1975 [56] where the range $10^{-3}<m_{H}<50 \mathrm{GeV}$ was considered. At that time most of the high mass SM particles were not discovered and therefore the results suffer from huge uncertainties. Before the LHC start, the strongest constraints were coming from LEP2 experiments with $m_{H}>114 \mathrm{GeV}$, Tevatron experiments, 


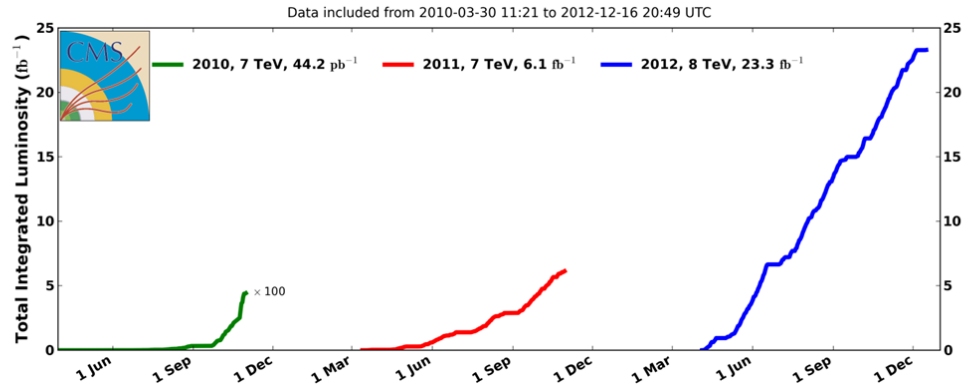

Fig. 10 LHC luminosity recorded by the CMS experiment.

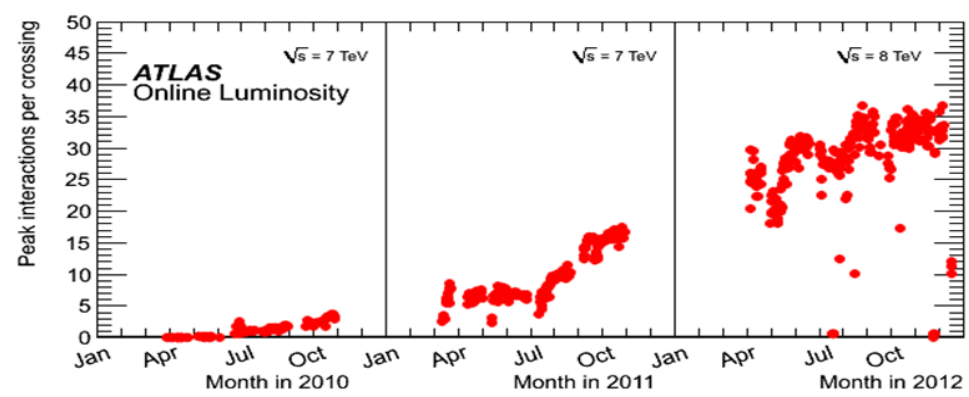

Fig. 11 Corresponding number of pile-up events as a function of time.

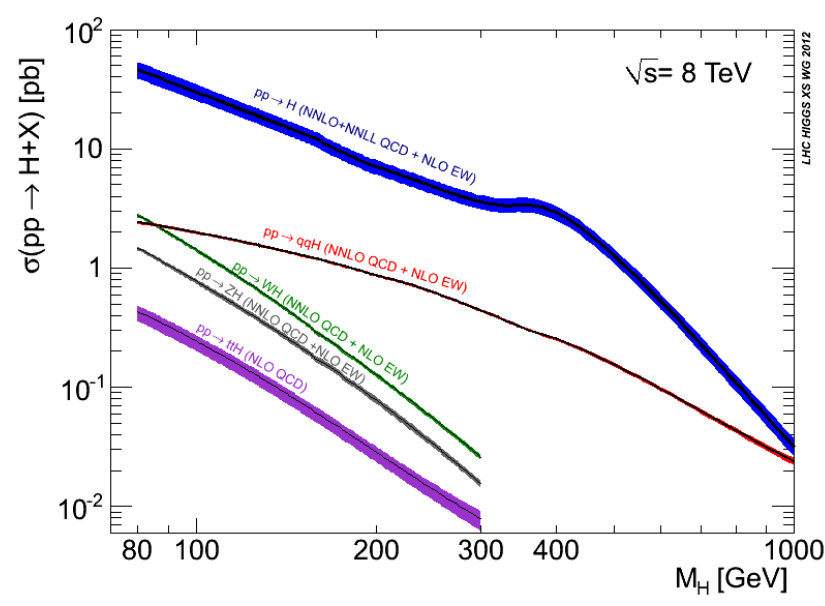

Fig. 12 SM Higgs production cross-section at LHC [54.

$160<m_{H}<175 \mathrm{GeV}$, and the unitarisation of the $W W$ scattering, $m_{H}<800 \mathrm{GeV}$.

Therefore in 2010, the allowed mass range for the Higgs boson search was restricted to less than one order of magnitude, with a preference for the "low" mass region, $\sim 100 \mathrm{GeV}$, coming from the EW fit (Section 2.2). The ATLAS and CMS searches were therefore divided in two categories: low mass searches where the discovery channels are $H \rightarrow \gamma \gamma(0.05 \mathrm{pb})$ and $H \rightarrow Z Z^{*} \rightarrow 4 l$ $(0.003 \mathrm{pb})$ and high mass searches where the discovery channels are $H \rightarrow W W^{(*)} \rightarrow 2 l 2 \nu(0.04 \mathrm{pb})$ and $H \rightarrow$

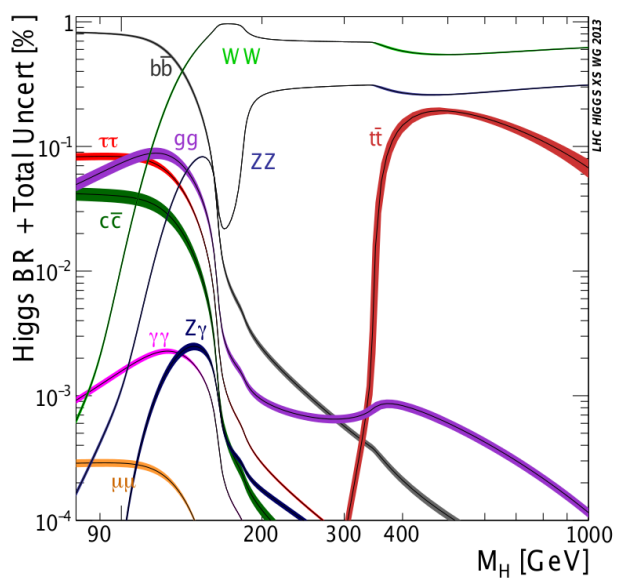

Fig. 13 SM Higgs production decay as a function of its mass [55].

$Z Z^{(*)} \rightarrow 4 l, 2 l 2 \nu(0.002 \mathrm{pb})$. In both cases $l=e, \mu$. Numbers in brackets indicate the cross-section values for $m_{H}=125 \mathrm{GeV}$ for low masses, and $m_{H}=500 \mathrm{GeV}$ for high masses.

As shown in Fig. 14, the $H \rightarrow \gamma \gamma$ channel can only been obtained by considering one-loop diagram in the production and the decay. This process, absent at tree-level, relies on the presence of virtual particles, i.e. discovering a signal in this channel will be a tri- 
umph for quantum field theory 4 . In this channel, the experimental challenge consists in reducing the contribution from jet faking a photon by a factor $\sim 10^{4}$, possible thanks to the high granular calorimeter. When achieved, the non-resonant SM $\gamma \gamma$ production becomes the dominant and irreducible background. A mass peak can then be searched for by fitting the side bands. With $10 \mathrm{fb}^{-1}$ of data, a clear peak with a significance above $4 \sigma$ was observed at $\mathrm{m}_{\gamma \gamma} \sim 126.5 \mathrm{GeV}$ by ATLAS, see Fig. 15 38. Similar results were obtained by the CMS experiment 39.

The $H \rightarrow Z Z^{*} \rightarrow 4 l$ channel, shown in Fig. 16 , is dominated by muon final states $(4 \mu$ and $2 e 2 \mu)$ and therefore almost background free, see Fig. 17. The experimental challenge is to maximize the coverage of the four leptons and master the lepton energy calibration. The latter can be cross-checked with on-shell $Z \rightarrow l^{+} l^{-}$ events, where one of the lepton radiates a photon that later converts to $l^{+} l^{-}$giving a peak at $90 \mathrm{GeV}$. With $10 \mathrm{fb}^{-1}$ of data, a clear excess is observed over the background estimation at $125.5 \mathrm{GeV}$ in CMS [39] as well as in ATLAS 38.

When combining both channels, the excess of events observed above the expected background around a mass of $125 \mathrm{GeV}$ has a local significance of $5 \sigma$ for both ATLAS and CMS. Furthermore, the production and decay of this particle is consistent with the SM Higgs boson within uncertainties.

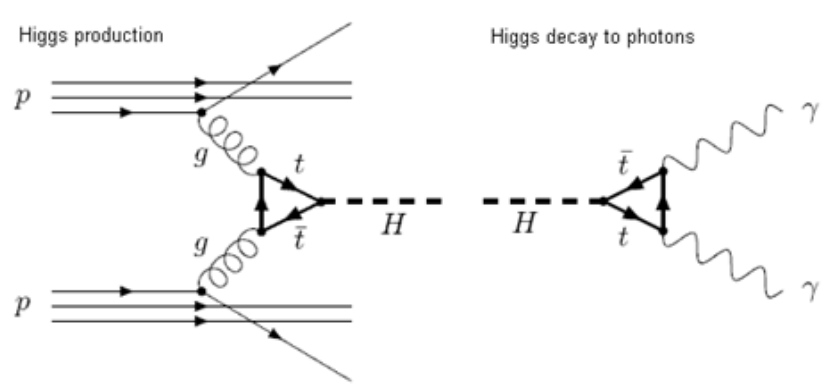

Fig. 14 Leading Feynman diagrams for the $H \rightarrow \gamma \gamma$ channel.

\subsubsection{Properties of the Higgs boson}

With the full run I statistics, it is already possible to study in some details the properties of this new particle. In particular, its mass can be determined at $0.5 \%$ precision by both experiments, i.e. more precisely than any quark mass in the SM. Current values are $125.4 \pm 0.4 \mathrm{GeV}$ and $125.0 \pm 0.3 \mathrm{GeV}$ for ATLAS and

${ }^{4}$ For these reasons, this channel is also sensitive to new particles in the loops.

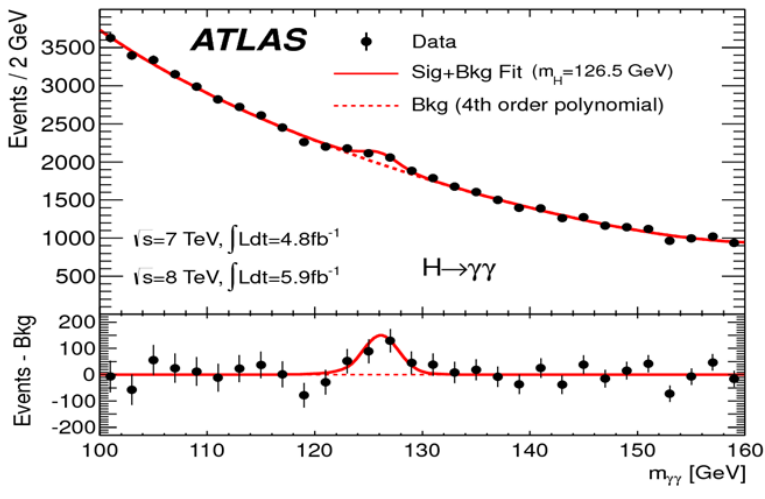

Fig. 15 Invariant mass of diphoton at the time of the discovery 38.

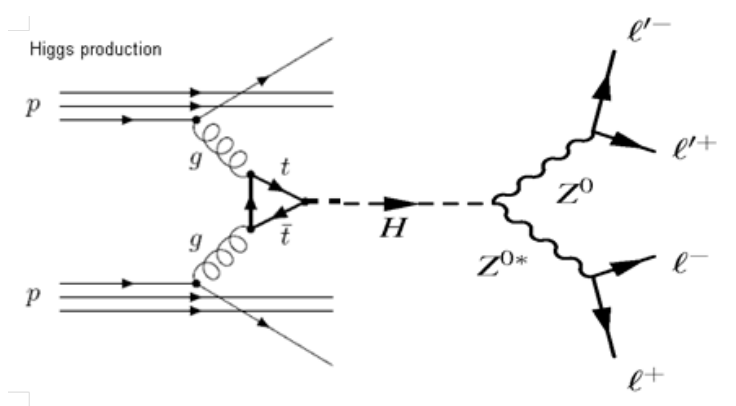

Fig. 16 Leading Feynman diagrams for the $H \rightarrow Z Z^{*} \rightarrow 4 l$ channel.

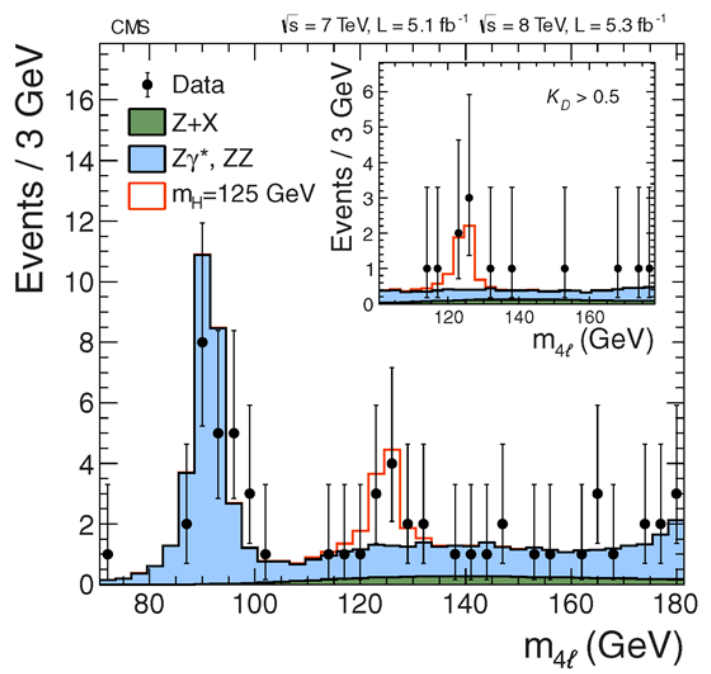

Fig. 17 Invariant mass of four-lepton at the time of the discovery 39]. 
CMS respectively [57,58, in agreement with the Higgs boson mass predicted by the EW fit, see Fig. 7 .

The spin of the new particle can be inferred from first principles and the observation of $H \rightarrow \gamma \gamma$ and $H \rightarrow$ $Z Z^{*} \rightarrow 4 l$ decays. The $\gamma \gamma$ final state forbids $J=n / 2$ (angular momentum conservation), strongly disfavors $J=1$ since on-shell vector boson can not decay to two massless photons [59,60] and disfavors $J=2$ since $\operatorname{BR}(H \rightarrow$ $\gamma \gamma) / \mathrm{BR}\left(H \rightarrow Z Z^{*} \rightarrow 4 l\right) \sim 0.1$ is hardly reproducible in graviton-inspired models. Assuming $J=0$, the parity of the new boson can be probed by looking at angular distribution of the four leptons coming from the Higgs decay. A negative parity is excluded at $99.6 \% 61$ favoring $J=0^{+}$as for the SM Higgs.

Once the Higgs mass is known, all Higgs couplings can be computed within the SM. The four production modes times six decay modes from Fig. 12 and 13 can be explored in principle, apart from $H \rightarrow g g, c \bar{c}$ which are not accessible because the background level is too high at LHC. To test the compatibility of these modes with the SM it is convenient to introduce coupling scale factors $\kappa$ defined as 62]:

$$
\begin{aligned}
& \sigma \times \mathrm{BR}(j j \rightarrow H \rightarrow i i)= \\
& \sigma_{\mathrm{SM}}(j j \rightarrow H) \times B R_{\mathrm{SM}}(H \rightarrow i i) \times \frac{\kappa_{i}^{2} \kappa_{j}^{2}}{\kappa_{H}^{2}} \sim \frac{\Gamma_{i} \Gamma_{j}}{\Gamma_{H}}
\end{aligned}
$$

Since at LHC only the ratio of partial width can be measured (no sensitivity to $\kappa_{H}$ since $\Gamma_{H}=4 \mathrm{MeV}$ ), there are 5 relevant $\kappa$ parameters: $\kappa_{t}, \kappa_{\tau}, \kappa_{b}$ for fermions and $\kappa_{W}, \kappa_{Z}$ for gauge bosons. Loop induced couplings $\kappa_{\gamma}$ and $\kappa_{g}$ are expressed with the previous parameters if the SM structure is assumed. Results are shown in Fig. 18 [58, where a very nice agreement between the SM predictions and experimental measure can be seen, even if uncertainties are still large. Looking at the coupling strength as a function of the particle mass, Fig.19. exhibits the very peculiar behavior of the SM Higgs.

All these measurements give a strong feeling that the boson discovered by ATLAS and CMS is an elementary scalar particle matching the Higgs boson of the Standard Model. At least this option has currently the highest probability and this will be our assumption for the remaining part of this section. For simplicity, we will use $m_{H}=125 \mathrm{GeV}$ and $\lambda=m_{H}^{2} /\left(2 v^{2}\right)=0.13$ and also assume that there is no new physics up to the Planck mass. These two hypotheses (no new physics and SM Higgs boson) will be rediscussed in details in Section 2.5. To conclude on LHC Higgs results, it is very important to mention that using all the statistics from run I and combining $H \rightarrow Z Z \rightarrow 4 l, 2 l 2 \tau, 2 l 2 \nu$ the presence of another SM Higgs-like boson is excluded below $1 \mathrm{TeV} 63$.

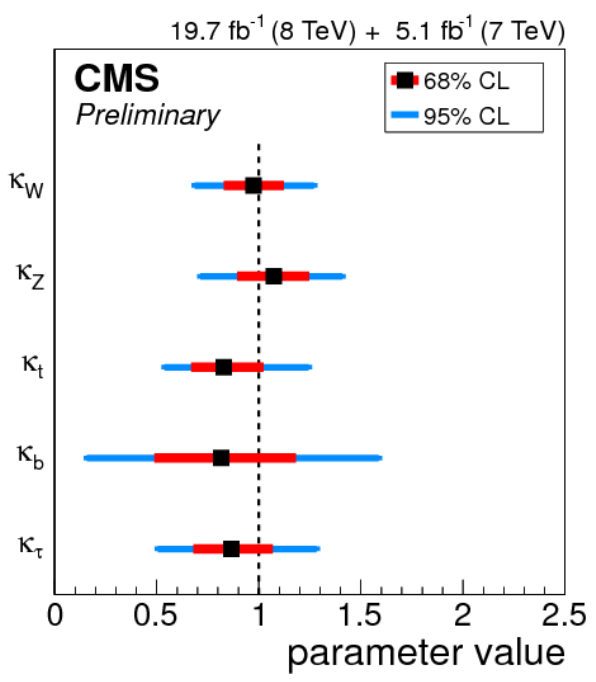

Fig. 18 Summary of the fit results for the generic fiveparameter model (see text) compared to the predictions from the SM shown with dotted line 58. Measurements are compared with the SM prediction (dotted line).

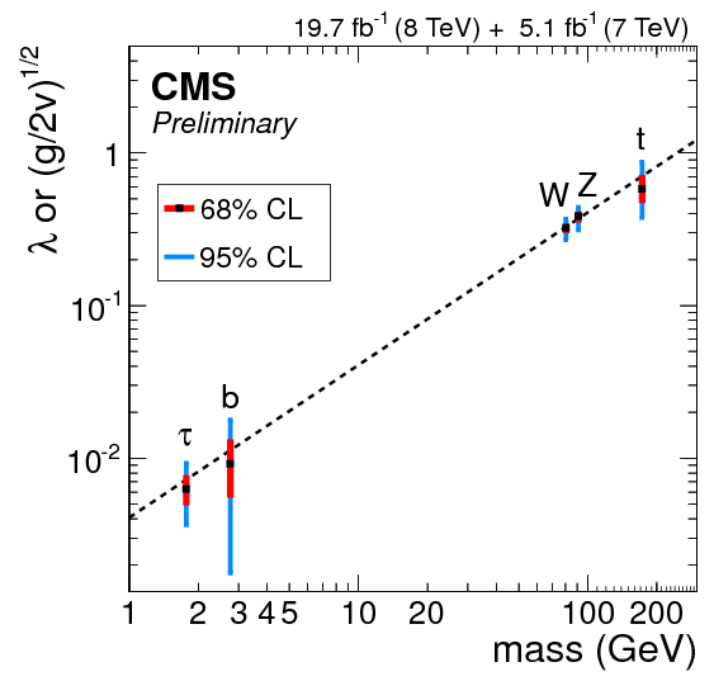

Fig. 19 Coupling strenght as a function of particle mass 58 . For fermions, the coupling strenght is $\lambda=\lambda_{Y}$, the fitted Yukawa coupling. For $V$ bosons, the coupling strenght is $\left(g_{V} /(2 v)\right)^{1 / 2}$ where $g_{V}=2 M_{V}^{2} / v$. Measurements are compared with the SM prediction (dotted line).

\subsubsection{Higgs and the Early Universe}

As already mentioned, the only way to probe experimentally the very first instant of the universe presently comes from particle physics experiments. However it should be said (even if it may sounds trivial) that these experiments do not reproduce all the conditions of the early universe, in particular there is no Hubble expansion and the matter is not in a hot plasma state with 
density $\rho$ and temperature $T$ connected by $\rho \propto T^{4}$. With these caveats in mind, let us illustrate the importance of the SM Higgs boson discovery on the early universe. For all these reasons, the Higgs mechanism occupies a central place in the early universe.

First, the Higgs mechanism provide a mass to all elementary fermions. Without this mechanism, electron will be massless and give macroscopic atoms, see eqn (1). Similarly massless quarks will prevent the atom to form since the proton can be more massive than the neutron (i.e. the neutron will be stable and the proton will decay to neutron, electron and neutrino). Note that $S U(2)_{L} \otimes U(1)_{Y}$ will still be broken at $\Lambda_{\mathrm{QCD}} \sim 0.2$ $\mathrm{GeV}$ and will give massive gauge bosons of $30 \mathrm{MeV}$, i.e. weak interaction will be strong 64.

Second, the form of the Higgs potential and the exact value of $m_{H}$ could have dramatically changed the universe that we know. The high temperature expansion of the Higgs potential at one-loop level can be written to a constant [65]:

$V(\phi, T)=a_{T} \phi^{2}+b_{T} \phi^{3}+c_{T} \phi^{4}$

The $a_{T}, b_{T}$ and $c_{T}$ parameters are defined as $\left(m_{t}^{4} \gg\right.$ $m_{W}^{4}, m_{Z}^{4}$ is assumed)

$a_{T}=a\left(T^{2}-T_{\mathrm{EW}}^{2}\right) \simeq \frac{m_{t W Z}^{2}}{4 v^{2}}\left(T^{2}-T_{\mathrm{EW}}^{2}\right) \simeq 0.33\left(T^{2}-T_{\mathrm{EW}}^{2}\right)$

$b_{T}=b T=-\frac{m_{Z}^{3}+2 m_{W}^{3}}{\sqrt{2} \pi v^{3}} T \simeq-0.027 T$

$c_{T}=\frac{m_{H}^{2}}{2 v^{2}}-\frac{3 m_{t}^{4}}{2 \pi^{2} v^{4}} \ln \frac{T}{m_{t}} \simeq \frac{m_{H}^{2}}{2 v^{2}} \simeq \lambda \simeq 0.13$

where $m_{t W Z}^{2}=2 m_{t}^{2}+2 m_{W}^{2}+m_{Z}^{2} \sim(285)^{2} \mathrm{GeV}^{2}$. It is interesting to note that, for a fixed temperature, the numerical values of these parameters depends only on $W, Z$, top and Higgs masses. The phase transition will occur at $T_{\mathrm{EW}}$ :

$$
\begin{aligned}
& T_{\mathrm{EW}}^{2}=\frac{1}{2 a}\left(m_{H}^{2}+\frac{3 m_{t}^{4}}{2 \pi^{2} v^{2}}\right)=\frac{1}{2 a}\left(m_{H}^{2}+\Delta m_{H}^{2}\right) \\
& \simeq 1.5\left(m_{H}^{2}+(47 \mathrm{GeV})^{2}\right)
\end{aligned}
$$

Note that neglecting the temperature and the radiative correction terms $a_{T} \rightarrow-a T_{\mathrm{EW}}^{2}=-m_{H}^{2} / 2, b_{T} \rightarrow 0$ and $c_{T} \rightarrow \lambda$ giving back eqn (2). For $T>T_{\mathrm{EW}}$, the universe is symmetric around $\phi_{\min }=0$ since $a_{T}>0$, and SM particles are massless. At the phase transition, $T=T_{\mathrm{EW}}, a_{T}=0$ and the potential shape depends only on $b$ and $\lambda$. If $|b| \geq \lambda / \sqrt{3}$, i.e $m_{H}<75 \mathrm{GeV}$, a second minimum of the potential appears at some critical temperature close to $T_{\mathrm{EW}}$ and its depth is equivalent to the minimum at $\phi_{\min }=0$. A first order transition could then start by quantum tunneling, see Fig. 20. For long, it was a nice explanation for baryogenesis, called EW baryogenesis, since CP violation on bubble surface could froze due to the phase transition 66,67 68 but this case is now excluded by the Higgs discovery at $m_{H}=125 \mathrm{GeV}$. In contrast we are in the case $|b|<\lambda / \sqrt{3}$, i.e $m_{H}>75 \mathrm{GeV}$, where the transition between the two minima is smooth and play no role in the baryogenesis. The term $\phi^{3}$ can be neglected and the only minimum of the potential, at tree level and low temperature, is located at $\phi_{\min }=\left(a T_{\mathrm{EW}}^{2} / \lambda\right)^{1 / 2}=v$, as shown in Fig. 21.

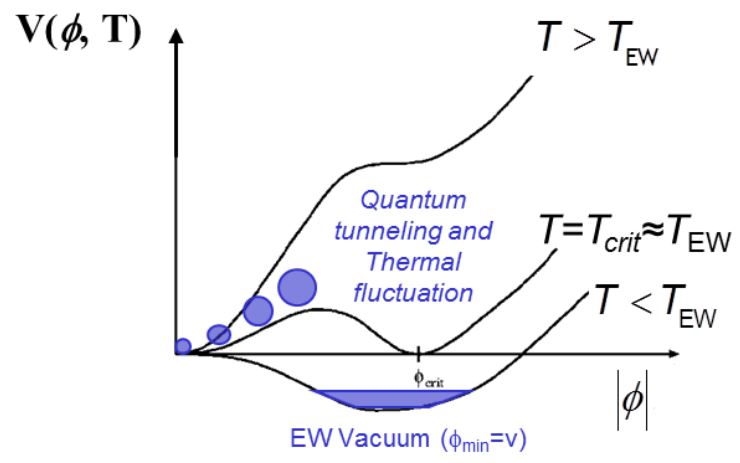

Fig. 20 Configurations of the Higgs potential with $m_{H}<$ $75 \mathrm{GeV}$.

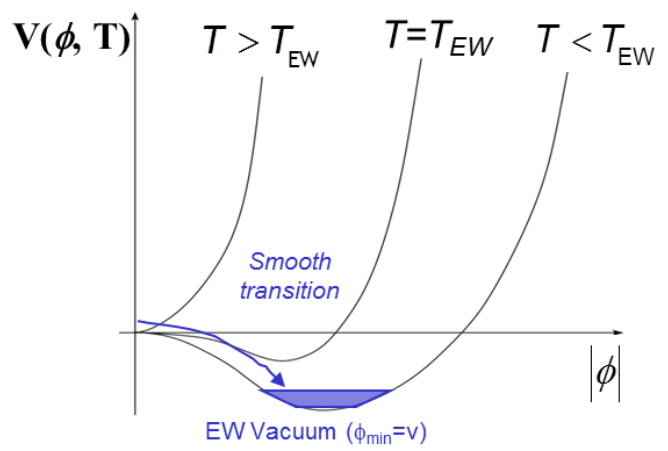

Fig. 21 Configurations of the Higgs potential with $m_{H}>$ $75 \mathrm{GeV}$.

Third, the discovery of an elementary scalar field is of primordial importance. The cosmological observations of recent decades revealed that the universe expanded with acceleration on two different stages of its evolution: in the very beginning and at present time. The former is presently described by the theory of inflation 69,70,71,72, the cornerstone of the Standard Model of cosmology. In this short period of time $\left(t=10^{-36}\right.$ $10^{-33} \mathrm{~s}$ ), the matter content of the universe must be dominated by a fluid with negative pressure, described 
by quantum field theory (given the high energy). The prototype is a scalar field $\phi$, called inflaton, since this is the only possibility compatible with symmetries implied by the cosmological principle. It is therefore very natural to ask whether the Higgs can play the role of the inflaton. For this to happen, it is mandatory to add a term $\xi \phi^{\dagger} \phi R$ to the Lagrangian [73, coupling non minimally the Higgs field to gravity, where $R$ is the Ricci scalar and $\xi$ is a dimensionless coupling constant generally large, $\mathrm{O}\left(10^{4}\right)$. In this context, the dimensionless quantity $\Psi=\sqrt{\xi} \phi / M_{P l}$ can control the cosmology:

$-\Psi \gg 1$ implies $\phi \gg M_{P l} / \sqrt{\xi}$, and the slow-roll inflation takes place.

- $\Psi \sim 1$ implies $\phi=M_{P l} / \sqrt{\xi}$, inflation ends and $T_{R} \geq$ $10^{13} \mathrm{GeV}$

- $\Psi \ll 1$ implies $\phi \ll M_{P l} / \sqrt{\xi}$ gets a vev as discussed earlier.

This is a seductive approach able to make predictions at two-loop level 74, 75, 76. Those predictions can be compared to precise experimental cosmological and particle physics measurements. For example, Higgs inflaton predictions match perfectly the latest Planck data [7]. It is however fair to mention that there is still some debate in the theory community about its validity [78].

Let us conclude this section by briefly mentioning the link between the Higgs boson and the cosmological constant. Latest Planck results $\left(\Omega_{v a c} \sim 0.7\right)$ confirmed that acceleration is taking place at present. It is therefore tempting to identify the constant energy density of the vacuum $\left(\rho_{\text {vac }}\right)$ to the EW vacuum. Plugging the vacuum configuration $\langle\phi\rangle=\frac{1}{\sqrt{2}}\left(\begin{array}{l}0 \\ v\end{array}\right)$ in eqn 2 gives, $\rho_{v a c}=-m_{H}^{2} v^{2} / 8 \sim-10^{8} \mathrm{GeV}^{4}$, highly incompatible with the cosmology measurements $\rho_{\text {vac }}=\Omega_{\text {vac }} \rho_{c r i} \sim$ $10^{-48} \mathrm{GeV}^{4}$. This is the cosmological constant problem [79]. For recent updates, see [80,81.

\subsection{The Higgs discovery calls for New Physics?}

The present situation in Particle Physics is paradoxical. On one hand, the Standard Model is amazing successful to describe all experimental data and since 40 years no significant deviation has been detected. On the other hand, it is plagued by many theoretical problems: very different mass and mixing for the 12 fermions (flavor problem), fine-tuned parameters $\left(m_{H}\right.$, see below, and $\left.\Theta_{Q C D}\right)$ and no GUT-scale unification of forces. Moreover the SM does not include gravity and consequently can not describe any large scale or very high energy phenomena. Finally, it does not have any good dark matter candidates (Section 3.4). Therefore the Standard Model can not be the ultimate theory and should be valid only up to a certain scale $\left(\Lambda_{N P}<M_{P l}\right)$.
Before going further, it is interesting to check whether the Higgs potential could survive up to $M_{P l}$ in the absence of any new physics. For very high energies, the Higgs potential can be approximate to $V=\lambda \phi^{4}$, i.e. the potential will only depend on the Higgs self coupling $\lambda$. If $\lambda \sim 0$, the EW vacuum is metastable and could become unstable if $\lambda<0$ which gives the lower bounds of Fig. 22, as of 2009 82. Today we know that $m_{H} \simeq 125 \mathrm{GeV}$, which means that the instability bound is crossed at $\Lambda \sim 10^{10} \mathrm{GeV}$. This is may be a hint on the presence of new physics at or before this scale. Pushing further to the Planck scale gives an intriguing result: the vacuum is metastable at $2 \sigma$ level, i.e. its lifetime is greater than the age of the universe, as illustrated in Fig. 23 83. It is noteworthy that the result is now driven by the top mass precision.

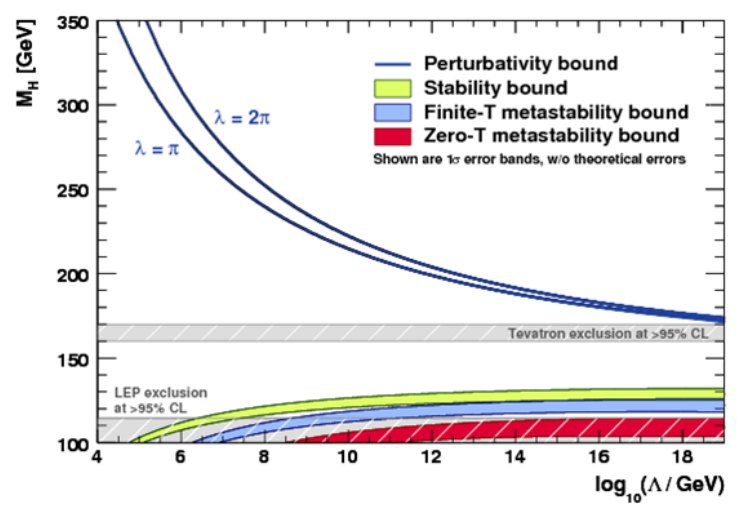

Fig. 22 Stability of the EW vacuum as a function of $\Lambda_{N P}$ before the Higgs discovery 82 .

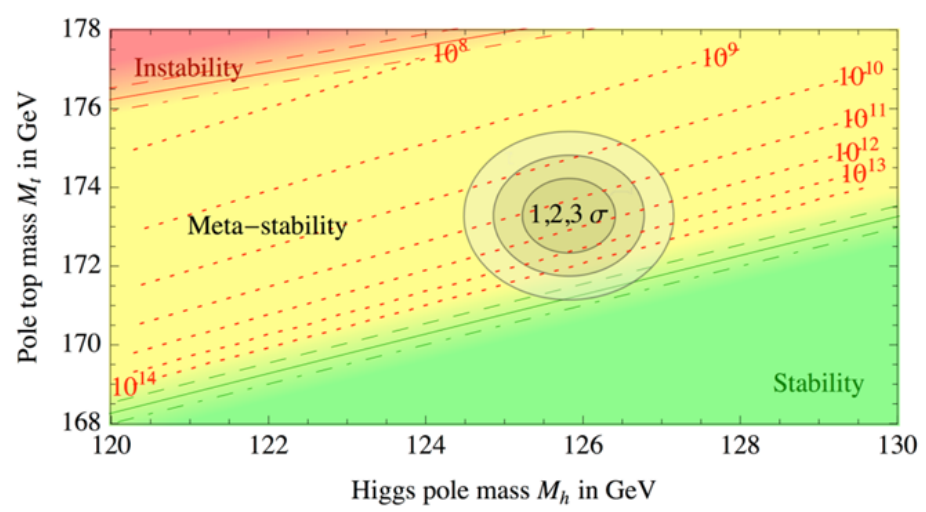

Fig. 23 Stability of the EW vacuum as a function of $\Lambda_{N P}$ after the Higgs discovery 83 .

Despite this intriguing result, solving the long list of SM problems discussed above requires the introduction of new particles, generally predicted by theories beyond 
the Standard Model (BSM). The most severe problem is coming from the fine-tuning of the Higgs mass and is called the hierarchy or naturalness problem 84,85. Hierarchy problem because one has to explain the extreme weakness of the gravity at short distance, reflected in the ratio between the Fermi and the Newton constants $\left(G_{F} \hbar^{2}\right) /\left(G_{N} c^{2}\right) \sim 1.7 \times 10^{33}$. It can be reformulated in terms of the Higgs mass divergence at high energy since $G_{F} \propto m_{H}^{-2}$, see eqn (3). Here, the problem is that an elementary spin- 0 particle mass is not protected by any symmetry unlike massive spin- 1 and spin- $1 / 2$ particles which are protected by broken gauge and chiral symmetries, respectively. As a massive scalar couples to all virtual particles present in the vacuum with an energy $E\left(\Lambda_{N P}=\max (E)\right)$, its radiative corrections can be expressed as [86]:

$$
\begin{array}{r}
\Delta m_{H}^{2}=\frac{3 G_{F}}{4 \sqrt{2} \pi^{2}}\left(4 m_{t}^{2}-2 m_{W}^{2}-m_{Z}^{2}-m_{H}^{2}\right) \Lambda_{N P}^{2}= \\
\kappa \Lambda_{N P}^{2} \simeq 0.05 \Lambda_{N P}^{2}
\end{array}
$$

This is the naturalness problem: $m_{H}^{2}$ requires a very high adjustment between the bare mass of the Higgs, $\left(m_{H}\right)_{0}^{2}$ and $\Delta m_{H}^{2}$. For $\Lambda_{N P}=M_{P l}$ this will look like: $m_{H}^{2}=36127890984789307394520932878928933023$ 36127890984789307394520932878928917398.

To restore naturalness, three main possibilities have emerged:

- Add a new broken symmetry between fermion and boson, called supersymmetry (SUSY), which protect the Higgs mass with new weakly coupled particles;

- Assume that Higgs is not elementary but a strongly coupled composite particle, requiring a reformulation of the problem;

- Assume extra spatial dimensions where gravity propagates in, which explains the weakness of gravity in our 4D world.

All these BSM theories predict new particles, partners of $t, W, Z$ and sometimes Higgs, below few TeV to dump the Higgs mass quadratic divergence. Generally a new conserved quantum number (parity) is attached to these new particles offering good candidate for dark matter 87. Moreover, assuming that naturalness is a guiding principle of Nature, which worked amazingly well in particle physics during the $\mathrm{XX}^{\text {th }}$ century 86 , 42 , it is possible to guess $\Lambda_{N P}$. For example requiring $\Delta m_{H}^{2}<m_{H}^{2}$ gives $\Lambda_{N P}<550 \mathrm{GeV}$, i.e. new physics directly accessible at LHC. Aside from a direct detection, the presence of new physics close to the EW scale could also cause observable deviations in all EW precision measurements (including now Higgs couplings) with respect to the SM predictions.
For all these reasons, signatures predicted by "natural" theories are guiding the new physics searches at LHC and are discussed in details in Section 3. Of course other attempts have been made to build BSM theories regardless of the naturalness argument. For example starting from the SM particle structure, it is reasonable to expect other quark and lepton generations and/or extra bosons $\left(Z^{\prime}, W^{\prime}, g^{\prime}\right)$ as well as other Higgs bosons $(n>1$ doublets instead of the minimal $n=1$ SM solution, or even triplets). Right-handed neutrinos, new symmetry between bosons and fermions or leptons and quarks could also be imagined. Pushing even further, asymmetric left and right-handed chiral multiplets could be part of a more general multiplet at GUT scale. Finally in order to reduce the number of SM parameters, a high energy unification of forces could be envisaged and/or mechanisms could be developed to explain SM parameter values and in particular the quantized value of electric charge giving $Q(e)+2 Q(u)+Q(d)=Q(u)+2 Q(d)=0\left(10^{-21}\right)$. Natural theories generally integrate or were build upon these ideas. Most of these SM extensions can be seriously challenged by LHC results as foreseen before LHC start 88 . Section 3 presents now in detail the status of these theories in light of LHC run I results. 


\section{Beyond Standard Model and Cosmology}

LHC gives an unique opportunity to explore the uncharted $0.1-\mathrm{O}(1) \mathrm{TeV}$ territory with $20 \mathrm{fb}^{-1}$ of data at $\sqrt{s}=8 \mathrm{TeV}$, where first hints from BSM physics are expected. This section presents in details the "eagerly awaited" results from the direct searches of BSM particles and discusses the impact on natural theories: SUSY in Section 3.1. extra dimensions and composite Higgs in Section 3.2 Other BSM models are briefly mentioned in Section 3.3 . The consequences of these searches on dark matter are presented in Section 3.4. Apart from LHC results probing the energy frontier, other areas of particle physics could provide evidence for BSM physics. This is the case of the neutrino sector, briefly reviewed in Section 3.5. which could give an elegant solution to matterantimatter asymmetry in the early universe. This section ends by presenting future prospects for particle physics in Section 3.6

\subsection{Supersymmetry searches at LHC}

Supersymmetry is the leading theory for physics beyond the Standard Model since it provides a solution to most of its shortcomings, including the hierarchy problem, and is based on very solid foundations 89 . It is the accomplishment of theoretical efforts started in the 70's and aiming at symmetrizing boson and fermion fields. This is done by creating chiral superfields, composed of a complex scalar and a spinor fermion, which transform in superspace via a new symmetry of space-time called supersymmetry. It was demonstrated that SUSY is the only non trivial extension of the Poincaré group, the space-time symmetry of the Standard Model. It was also realized that the commutators of two local SUSY transformations give a local translation: therefore local SUSY naturally implies gravity with two gauge fields, the graviton $G$ and the gravitino $\tilde{G}$, a step forward compare to the SM, which has no description of gravity. The SUSY phase space is huge and only the part can be probed at LHC is mentionned hereafter.

\subsubsection{Minimal SuperSymmetric Model (MSSM) and natural spectrum}

After a decade of theoretical work a realistic SUSY model, in form of the minimal SM extension that realizes $\mathrm{N}=1$ supersymmetry, was proposed at the beginning of the 80's [90]. This model predicts new particles, called sparticles, that are the superpartner of each SM particle in the chiral multiplets. A new quantum number, R-Parity, negative/positive for SUSY/SM particles, is created. The sparticles have therefore the same quantum numbers as their SM partners, except for the spin, half a unit of spin different, and R-Parity. A remarkable by-product is that these new particles allow for the unification of forces at the GUT scale, solving one the SM problem. The complete list of sparticles is given in Fig. 24 and detailed briefly below.

To give masses to up and down-type fermions, the SM Higgs sector needs to be extended by adding another $S U(2)_{L}$ complex doublet. As a result, 8 mass eigenstates exists: three neutral Higgses $\left(h^{0}\right.$, the lighter, $H^{0}$ and $A^{0}$ ), two charged Higgses $\left(H^{ \pm}\right)$and three Goldstone bosons $\left(G^{0}, G^{ \pm}\right)$'eaten' to give $W$ and $Z$ masses. Each of the neutral component of the doublet acquires a vev called $v_{u}$ and $v_{d}$ related by $v_{u}^{2}+v_{d}^{2}=v^{2}$ and $\tan \beta=v_{u} / v_{d}$, to get the correct $W$ and $Z$ masses.

The other new particles are the squarks $\tilde{q}$ and the sleptons $\tilde{l}$, spin-0 partners of the SM fermions. Similarly, Wino $\tilde{W}$, Bino $\tilde{B}$ and Higgsinos $\tilde{H}_{u, d}^{0, \pm}$ are the spin-1/2 superpartners of the EW bosons and mix to give EWKinos decomposed in 4 neutralinos $\tilde{\chi}_{1,2,3,4}^{0}$ (noted $\tilde{N}$ in Fig. 24) and 4 charginos $\tilde{\chi}_{1,2}^{ \pm}$(noted $\tilde{C}$ in Fig. 24 . To complete the list, colored gluinos $\tilde{g}$ and the gravitino $\tilde{G}$ are the partners of the gluon and graviton. Note also that left and right-handed fermions have two different SUSY partners $\tilde{f}_{L, R}$ that could mix to give mass eigenstates $\tilde{f}_{1,2}$ provided the SM partner is heavy enough, like in the third generation. With this set-up the number of fermions and bosons is equalized and among others 21 new elementary scalar particles are predicted. This generally explains the lower SUSY production cross-section compare to other BSM theories and makes non-colored sparticle discovery particularly challenging. Since each SM particle and its superpartner belong to the same multiplet, the sparticle decay generally involves the SM partner. However due to the high number of new particles many different decays are possible depending on the sparticle mass spectrum. This other reason explains also why the sparticle discovery is an extremely challenging task.

Even if it is not the only possible realization of SUSY, MSSM still serves as a reference for today's searches since it provides a very elegant solution to the hierarchy problem: if in each SUSY multiplet sparticle and particle have the same mass, the coupling of sparticles with the Higgs removes exactly the quadratic mass divergence, i.e $\kappa=0$ in eqn (9). Even if the mass degeneracy is disproved experimentally, the introduction of sparticles replaces the quadratic divergence by a logarithmic divergence of the form $\left(m_{\tilde{f}}^{2}-m_{f}^{2}\right) \ln \left(\Lambda_{N P} / m_{H}\right)$, a substantial gain for naturalness. Imposing a natural theory will therefore imply that all heavy particles entering eqn (9) have masses close to their SM partners: stop should be close to the top quark mass, Wino and 
Bino close to $W$ and $Z$ masses and Higgsinos, governed by the $\mu$ parameter appearing at tree level, very close to the Higgs mass. Since stop is a scalar, its mass will quadratically diverge unless it is protected by a $\mathrm{O}(\mathrm{TeV})$ gluino appearing in the loop $\tilde{t}_{1} \rightarrow \tilde{g} t \rightarrow \tilde{t}_{2}$. Since the left-handed bottom is part of the SM doublet including left-handed top, the left-handed sbottom should also be light. Finally because of the lower Yukawa couplings of leptons and other quarks, sleptons and other squarks are less constrained. They are even required to be heavy and degenerate to avoid too high $\mathrm{CP}$ violation and/or Flavor Changing Neutral Current (FCNC) already excluded experimentally. Figure 25 shows natural SUSY particle mass spectra integrating these constraints and giving less than $10 \%$ tuning. As before 2010 no exploration of this natural spectrum was possible, the LHC experiments were ideally placed to discover or disprove the presence of these new particles.

The other consequence of the non-mass degeneracy of particle and sparticles is that SUSY should be broken. However, unlike for the EW symmetry, it was realized at the end of 70's that SUSY can not be spontaneously broken. Instead it is softly broken in a hidden sector that communicates to the visible sector via a messenger that could be gravity (supergravity (SUGRA)like models) or gauge bosons (gauge-mediated SUSYbreaking (GMSB)-like models). For gravity mediation an alternative is that no tree-level coupling transmits the SUSY breaking and sparticles masses are generated by one or two loop diagrams (anomaly-mediated SUSY breaking (AMSB)-like models). In any case, the price to pay for the soft SUSY breaking is the addition of 105 new parameters compared to the SM.

\begin{tabular}{|c|c|c|c|c|}
\hline Names & Spin & $P_{R}$ & Gauge Eigenstates & Mass Eigenstates \\
\hline Higgs bosons & 0 & +1 & $H_{u}^{0} H_{d}^{0} H_{u}^{+} H_{d}^{-}$ & $h^{0} H^{0} A^{0} H^{ \pm}$ \\
\hline squarks & 0 & -1 & $\begin{array}{cccc}\tilde{u}_{L} & \widetilde{u}_{R} & \widetilde{d}_{L} & \widetilde{d}_{R} \\
\widetilde{s}_{L} & \widetilde{s}_{R} & \widetilde{c}_{L} & \widetilde{c}_{R} \\
\tilde{t}_{L} & \widetilde{t}_{R} & \widetilde{b}_{L} & \widetilde{b}_{R}\end{array}$ & $\begin{array}{c}\text { (same) } \\
\text { (same) } \\
\widetilde{t}_{1} \tilde{t}_{2} \widetilde{b}_{1} \widetilde{b}_{2}\end{array}$ \\
\hline sleptons & 0 & -1 & $\begin{array}{ccc}\widetilde{e}_{L} & \widetilde{e}_{R} & \widetilde{\nu}_{e} \\
\tilde{\mu}_{L} & \widetilde{\mu}_{R} & \widetilde{\nu}_{\mu} \\
\widetilde{\tau}_{L} & \widetilde{\tau}_{R} & \widetilde{\nu}_{\tau}\end{array}$ & $\begin{array}{c}\text { (same) } \\
\text { (same) } \\
\widetilde{\tau}_{1} \widetilde{\tau}_{2} \widetilde{\nu}_{\tau}\end{array}$ \\
\hline neutralinos & $1 / 2$ & -1 & $\begin{array}{llll}\widetilde{B}^{0} & \widetilde{W}^{0} & \widetilde{H}_{u}^{0} & \widetilde{H}_{d}^{0}\end{array}$ & $\begin{array}{llll}\widetilde{N}_{1} & \widetilde{N}_{2} & \widetilde{N}_{3} & \widetilde{N}_{4}\end{array}$ \\
\hline charginos & $1 / 2$ & -1 & $\widetilde{W}^{ \pm} \widetilde{H}_{u}^{+} \widetilde{H}_{d}^{-}$ & $\tilde{C}_{1}^{ \pm} \widetilde{C}_{2}^{ \pm}$ \\
\hline gluino & $1 / 2$ & -1 & $\widetilde{g}$ & (same) \\
\hline $\begin{array}{c}\text { goldstino } \\
\text { (gravitino) }\end{array}$ & $\begin{array}{c}1 / 2 \\
(3 / 2) \\
\end{array}$ & -1 & $\widetilde{G}$ & (same) \\
\hline
\end{tabular}

Fig. 24 SUSY particles predicted by MSSM [90].

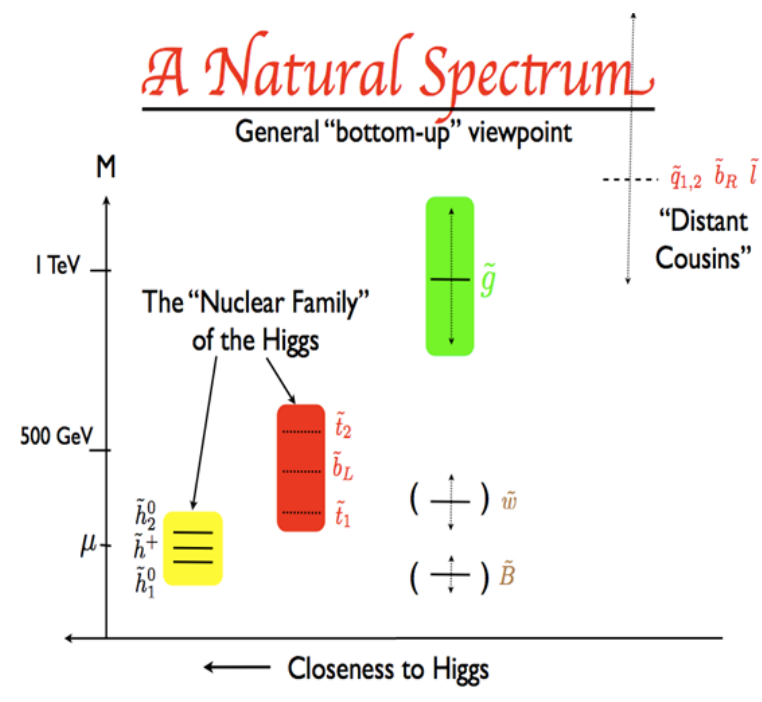

Fig. 25 Natural SUSY particle mass spectra giving less than $10 \%$ tuning 91 .

\subsubsection{Search strategy at $L H C$}

ATLAS and CMS have developed a rich and coherent program to discover SUSY particles which resulted in about 200 public analyses [92,93. The program mainly focuses on models where R-parity is conserved (RPC) since this is a simple way to prevent a too fast proton decay, and will provide a very good candidate for dark matter. This assumption has two important phenomenological consequences: first the lightest SUSY particle (LSP) is stable and corresponds to the massive lightest neutralino $\left(\tilde{\chi}_{1}^{0}\right)$ or the approximately massless gravitino $(\tilde{G})$ in SUGRA-like or GMSB-like models, respectively. Second, sparticles will be pair-produced at LHC. Highest cross-sections are expected from gluinogluino, squark-antisquark and gluino-squark production (strong SUSY): for a $1 \mathrm{TeV}$ gluino, $\sigma(\tilde{g} \tilde{g})=0.05$ pb giving 1000 events at LHC run I. This is typically 10 times the first and second generation squark-antisquark crosssection and 100 times the stop-antistop cross-section. EWKinos production cross-sections are much lower than strong SUSY. A $400 \mathrm{GeV}$ pair-produced chargino has also $\sigma=0.05 \mathrm{pb}$, typically 100 times more than pairproduced leptons of the same mass.

To clarify the presentation of the results, RPC searches are shown in sequence: $i$ ) gluinos, first and second generation squarks, $i i$ ) third generation squarks, $i i i)$ EWKinos and sleptons $i v$ ) a summary of RPC searches after run I. Searches for other signatures including R-Parity Violated (RPV), Long-lived particles or beyond MSSM solutions are then discussed. For conciseness and pedagogic reasons, only ATLAS results are reported since CMS obtained very similar results. A full ATLAS and 
CMS review can be found in [94. To be complete SUSY Higgs searches are first recalled. All limits below are reported at $95 \%$ Confidence Level (CL).

\subsubsection{SUSY Higgs searches}

The newly discovered Higgs boson may well be the lightest neutral Higgs of the MSSM $\left(h^{0}\right)$ since it possesses very similar properties as the SM one when $m_{A}^{2} \gg$ $m_{Z}^{2}$ and $\tan \beta>1$ (decoupling limit). Note, however, that $125 \mathrm{GeV}$ is close to the upper mass bound of possible lightest Higgs masses in MSSM and requires high stop masses, in tension with the natural SUSY spectrum. Extra neutral and charged Higgses, which preferentially couple to the most massive down-type fermions, are also actively searched. At the LHC, neutral Higgses are produced singly or accompanied by $b$-jet(s) and decay via $\tau^{+} \tau^{-}, b \bar{b}$ and more marginally $\mu^{+} \mu^{-}$. Charged Higgses with lower masses than the top quark predominantly appear in top decays $t \rightarrow b H^{ \pm} \rightarrow b \tau \nu$. On the other hand, charged Higgses with higher masses than the top quark are produced in association with top and bottom quarks and decay via $H^{ \pm} \rightarrow t b$.

Searches therefore focus on final states with $t, b$ and/or $\tau$. Most up-to-date searches for neutral Higgses [95, 96, as well as for charged Higgses 97] are interpreted in the $\left(\tan \beta, m_{A}\right)$ plane for neutral Higgses and $\left(\tan \beta, m_{H^{ \pm}}\right)$plane for charged Higgses, there being the relevant tree-level parameters since $m_{H^{ \pm}}^{2}=m_{A}^{2}+m_{W}^{2}$. Other SUSY parameters, entering via radiative corrections, are fixed to particular benchmark values, chosen to exhibit certain MSSM features. The most commonly used scenario, called $m_{h}^{\max }$ 98, maximizes the lightest neutral Higgs mass for a fixed $\tan \beta$ and large $m_{A}$, while the stop and sbottom masses are around $1 \mathrm{TeV}$. In this scenario, the null result on neutral Higgs searches can rule out models with $m_{A}<125 \mathrm{GeV}$ as well as large values of $\tan \beta(>5)$. For $m_{H^{ \pm}}<m_{t}, H^{ \pm}$masses are practically excluded below $160 \mathrm{GeV}$ for all $\tan \beta$ values while if $m_{H^{ \pm}}>m_{t}$, most of the $\left(\tan \beta, m_{H^{ \pm}}\right)$ plane is still not excluded. Those results favor neutral and charged SUSY Higgses with masses higher than $h^{0}$ mass, even if no model-independent limits yet exist.

\subsubsection{Direct searches of gluinos and first- and second-generation squarks}

At LHC, TeV-scale squarks and gluinos decay promptly in long decay chains containing mainly quark/gluon jets and the LSP. SUSY events are therefore characterized by multiple energetic jets as well as transverse missing energy $E_{T}^{\text {miss }}$ originated from the undetected LSP energies. Depending on the sparticle mass spectrum be- tween the squarks/gluinos and the LSP, charged lepton(s) and/or photons could also appear in the cascade. Since LHC is an hadronic machine, the experimental challenge is to reduce the multijet SM backgrounds by several orders of magnitude. The latter is mainly composed of QCD multijets $\left(\sigma \sim 10^{10} \mathrm{pb}\right), W / Z+$ jets $\left(\sigma \sim 10^{5} \mathrm{pb}\right)$, top $\left(\sigma \sim 10^{2} \mathrm{pb}\right)$, Dibosons $(\sigma \sim 50$ $\mathrm{pb})$ and eventually $t t W$ or $t t Z(\sigma \sim 0.1 \mathrm{pb})$. In all cases, the presence of jets and real or fake $E_{T}^{\text {miss }}$ could mimic the SUSY signal. On top of high initial kinematic cuts, the RPC Strong production analyses are based on very powerful discriminating variables, which exploit the main characteristics of the SUSY decay chain: the correlation between the scalar sum of the transverse energy of reconstructed objects, $H_{T}$, and the module of their vectorial sum, $E_{T}^{m i s s}$. Combinations of $E_{T}^{\text {miss }}$ and $H_{T}$ like in the effective mass variable, $m_{\text {eff }}=H_{T}+$ $E_{T}^{\text {miss }}$ 99, or the missing transverse momentum significance, $E_{T}^{\text {miss }} / \sqrt{H_{T}}$ could provide extra sensitivity. All these discriminating variables are consistently used in ATLAS for strong SUSY searches. They can be linked to some characteristic SUSY parameters like $M_{\mathrm{SUSY}}$, the mass of the highest colored object, $M_{\mathrm{LSP}}$, the LSP mass, and their difference $\Delta M$, see Fig. 26. Typically $m_{\mathrm{eff}}$ will peak at $1.8\left(M_{\mathrm{SUSY}}^{2}-M_{\mathrm{LSP}}^{2}\right) / M_{\mathrm{SUSY}}$ [100]. For open spectra $(\Delta M>\mathrm{O}(500) \mathrm{GeV})$, this value is well above the SM background which has no correlation between $E_{T}^{\text {miss }}$ and $H_{T}$, and therefore peaks at lower values, see Fig. 27. However for compressed spectra $(\Delta M<500 \mathrm{GeV}), m_{\text {eff }}$ loses his separation power and cut values should be relaxed.

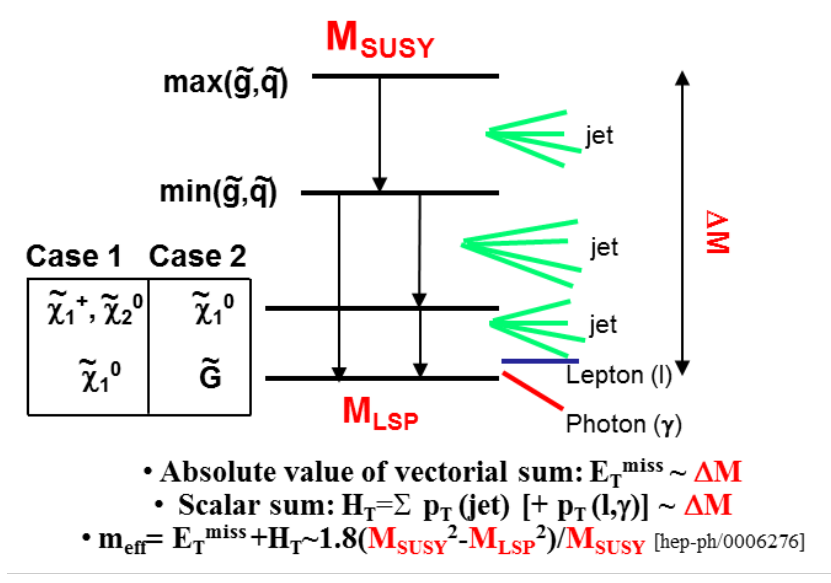

Fig. 26 Strategy of the ATLAS search for colored SUSY particles. The two cases shown in at the bottom of the SUSY spectrum correspond to the two considered LSP types 94].

SUGRA-like scenario can be investigated in the most inclusive way by considering lepton veto analyses. In 


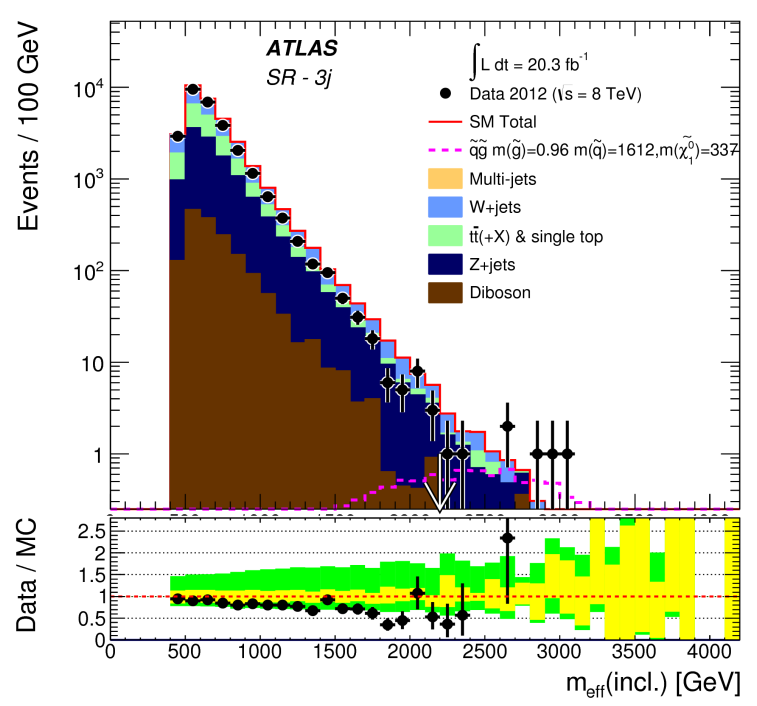

Fig. $27 m_{\text {eff }}$ distribution in one signal region of the inclusive search channel with no lepton [101.

this case, signatures with 2 to 6 jets can probe squarksquark (2 jets or more), squark-gluino (3 jets or more) or gluino-gluino (4 jets or more) production. Three signal regions (SRs) target the high $M_{\mathrm{SUSY}}$ and high $\Delta M$ by applying tight cuts on $m_{\text {eff }}>\mathrm{O}(1) \mathrm{TeV}$. Seven 'medium/loose' SRs cover more compressed spectra, by relaxing the cuts on $m_{\text {eff }}$. The absence of excess can be interpreted in a minimal SUGRA (mSUGRA) with 5 parameters (Fig. 28). The two most relevant parameters are the universal scalar (fermion) masses at GUT scale called $m_{0}\left(m_{1 / 2}\right)$ proportional to squarks (gluinos) masses at the EW scale as shown by the isolines. The other parameters are chosen to accommodate a 125 GeV Higgs mass. The top left part of the plot is dominated by squark-squark production best covered by the 4-jets tight SR (2 squark jets + initial and final radiation jets), while the bottom right part, is dominated by gluino-gluino, where 6-jets tight SR is best. It is also very interesting to interpret this results 'topologically', i.e. assuming that only few particles are accessible in the SUSY mass spectrum. Figure 29 show the limits obtained when considering only gluinos, mass-degenerate first/second generation squarks and the LSP. Decays of squarks and gluinos are then forced, with $100 \%$ branching ratio, via $\tilde{g} \rightarrow q \bar{q} \tilde{\chi}_{1}^{0}$ and $\tilde{q} \rightarrow q \tilde{\chi}_{1}^{0}$. In this case, mass limits are above $1.4 \mathrm{TeV}$ for gluinos and first/second generation squarks if LSP masses are below $400 \mathrm{GeV}$. For mSUGRA and topological models, squark and gluino with degenerate masses are excluded below $1.7 \mathrm{TeV}$ [101].

The most 'natural' decay of the gluino is $\tilde{g} \rightarrow t \tilde{t} \rightarrow$ $t t \tilde{\chi}_{1}^{0}$, see Fig. 25. This channel provides 4 tops plus high $E_{T}^{m i s s}$ final states but is only poorly covered by the previous analysis. An extra sensitivity can be obtained by

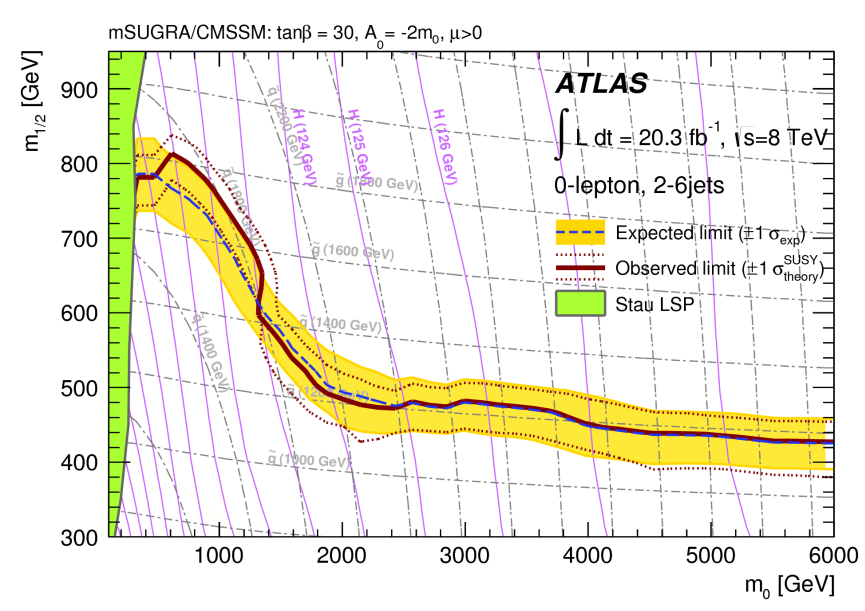

Fig. 28 SUSY limits from the inclusive search with no lepton in the minimal SUGRA scenario [101.

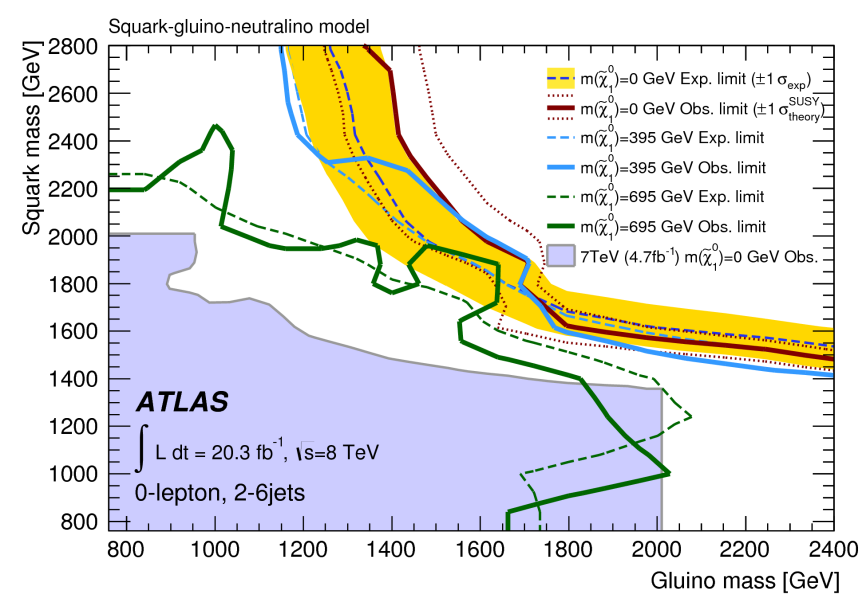

Fig. 29 Limits on topological models assuming only massdegenerate first/second generation of squarks, gluinos and the LSP 101.

considering final states not produced by the dominant $t \bar{t} \rightarrow W^{+} W^{-} b \bar{b}$ background, i.e. $\left.i\right)$ more than 6 jets and no lepton [102, ii) two same-sign leptons [103] or iii) 3 b-jets 104 with or without a lepton. The best sensitivity is obtained by the latter that can exclude gluino masses up to $1.4 \mathrm{TeV}$ for LSP masses below 500 $\mathrm{GeV}$ assuming a $100 \%$ branching ratio for the decay $\tilde{g} \rightarrow t \tilde{t} \rightarrow t t \tilde{\chi}_{1}^{0}$.

All described analyses probe high gluino and squark masses but generally requires open SUSY spectra. For more compressed ones, the $m_{\text {eff }}$ cut could be relaxed (medium and loose SRs of the no-lepton analysis) but even there, it is generally not possible to probe LSP masses above $500 \mathrm{GeV}$. To improve on this, asking for one soft electron or $\mu$ [105] or two same sign leptons is generally better. Experimental challenges drastically change: lepton triggers can be exploited and cuts on 
jet kinematics can be reduced. Lowering cuts on $E_{T}^{\text {miss }}$ and $m_{\mathrm{eff}}$ is possible since the multijet QCD background is naturally suppressed by the presence of isolated leptons. Finally other variables exists like the transverse mass $m_{T}$, built from lepton and $E_{T}^{\text {miss }}$, which efficiently reduce $t \bar{t}$ and $W+$ jets backgrounds by asking $m_{T}>$ $m_{W}$. These analyses are sensitive to LSP masses of $600 \mathrm{GeV}$, when the lepton(s) originates from an intermediate sparticle, located between squarks/gluinos and LSP like in $\tilde{\chi}_{1}^{ \pm} \rightarrow W^{ \pm}(\rightarrow l \nu) \tilde{\chi}_{1}^{0}$ or $\tilde{t} \rightarrow t \tilde{\chi}_{1}^{0} \rightarrow W^{ \pm}(\rightarrow$ $l \nu) b \tilde{\chi}_{1}^{0}$.

GMSB-like scenario are providers of SUGRA-like final states but also offers other experimental possibilities. In these models, LSP is the gravitino and final states are driven by the coupling of the Next to Lightest SUSY Particle (NLSP) to the LSP. A natural solution is that the NLSP is the lightest neutralino, $\tilde{\chi}_{1}^{0}$. In this case, depending on the mixing parameters, $\tilde{\chi}_{1}^{0} \rightarrow \gamma \tilde{G}$, $\tilde{\chi}_{1}^{0} \rightarrow Z^{0} \tilde{G}$ and $\tilde{\chi}_{1}^{0} \rightarrow h^{0} \tilde{G}$ could be opened giving for example $\gamma \gamma, \gamma Z^{0}(\rightarrow l l), \gamma h^{0}(\rightarrow b b)$ final states. These final states will be overlaid with jets coming from the gluino/squark cascade. Present limits generally exclude gluino masses below $1 \mathrm{TeV}$ and less stringent limits are obtained for squarks [106. However no $8 \mathrm{TeV}$ results are (yet) available.

\subsubsection{Direct searches for third-generation squarks}

Given the limits on the gluino masses, it is conceivable that strong SUSY production could be dominated by the direct production of stop or left sbottom. Compared to gluino pair-production, less complex final states with an enhanced presence of $b$-jet(s) are then expected.

The simplest signature is given by the direct lefthanded sbottom pair production, where $\tilde{b}_{L} \rightarrow b \tilde{\chi}_{1}^{0}$ : exactly two $b$-jets, no lepton and high $E_{T}^{\text {miss }}$. To take full advantage of the simple topology, $m_{\mathrm{eff}}$ is replaced by $m_{\mathrm{CT}}$ [107,108, which allows a better signal to background separation, as shown in Fig. 30. The reason is that two distinct end-points are obtained for signal, around $m_{\mathrm{CT}} \sim\left[M(\tilde{b})^{2}-M\left(\tilde{\chi}_{1}^{0}\right)^{2}\right] / M(\tilde{b})$, and for $t \bar{t}$ background, around $m_{\mathrm{CT}} \sim\left[M(t)^{2}-M(W)^{2}\right] / M(t) \sim 140$ $\mathrm{GeV}$. Figure 31 shows that sbottom masses below 650 $\mathrm{GeV}$ are excluded when $M_{\mathrm{LSP}}<200-300 \mathrm{GeV}$ [109], getting close to the upper bounds of the natural spectrum. Other sbottom decays $\tilde{b} \rightarrow t \tilde{\chi}_{1}^{ \pm}$and $\tilde{b} \rightarrow b \tilde{\chi}_{2}^{0}$, are covered by two same-sign leptons and $3 b$-jets analyses respectively and give generally slightly lower limits.

The case of the lightest stop $\tilde{t}_{1}$, the most pressing issue for the Higgs mass stability at high energy, is a bit more complex since the topology is even closer to the SM $t \bar{t}$. Generally the main difference arose from the higher expected $E_{T}^{\text {miss }}$ due to the presence of the

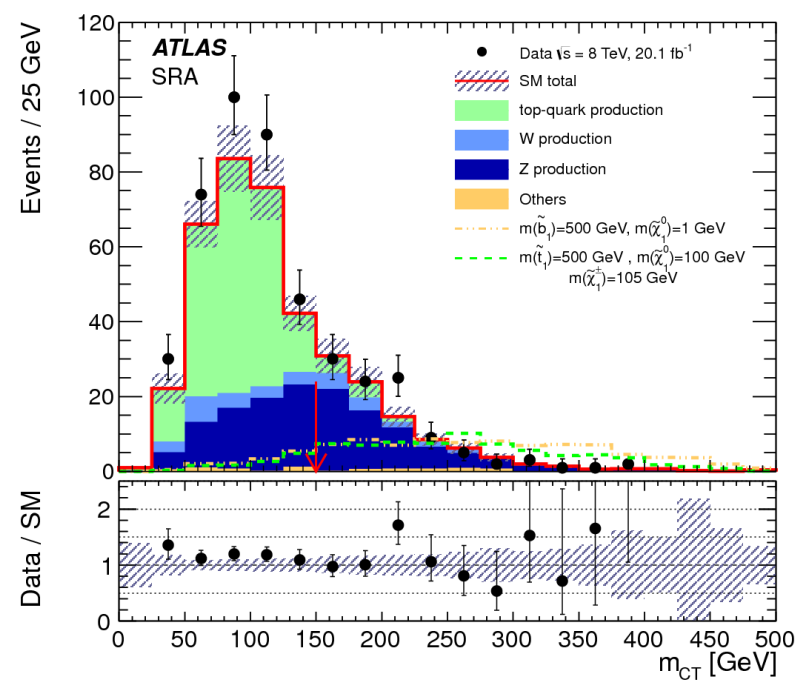

Fig. 30 Highlights from the direct sbottom searches : $m_{\mathrm{CT}}$ distribution [109].

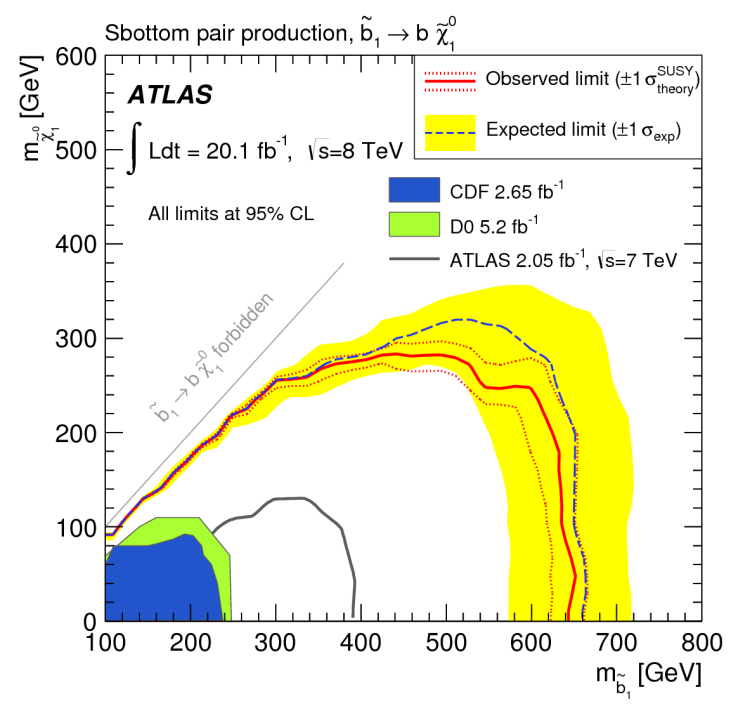

Fig. 31 Present limits in the sbottom-LSP plane in the case $\tilde{b}_{L} \rightarrow b \tilde{\chi}_{1}^{0}[109$.

LSP. The stop decays can be divided in two classes: $\tilde{t}_{1} \rightarrow t \tilde{\chi}_{1}^{0}, b W \tilde{\chi}_{1}^{0}, c \tilde{\chi}_{1}^{0}$ and $\tilde{t}_{1} \rightarrow b \tilde{\chi}_{1}^{ \pm} \rightarrow b W^{ \pm(*)} \tilde{\chi}_{1}^{0}$. The former is best covered by signatures with 0 -lepton +6 jets including $2 b$-jets 110 and 1 -lepton +4 -jets including $1 b$-jet [111] for $\tilde{t} \rightarrow t \tilde{\chi}_{1}^{0}$ decay, 2-leptons + jets for $\tilde{t} \rightarrow$ $b W \tilde{\chi}_{1}^{0}$ decay [112] and $2 c$-jets for $\tilde{t} \rightarrow c \tilde{\chi}_{1}^{0}$ decay [113]. As can be seen from the right part of Fig. 32 , a stop mass below $700 \mathrm{GeV}$ is excluded for $M_{\mathrm{LSP}}<100 \mathrm{GeV}$, apart from some holes around $m_{\tilde{t}_{1}} \sim m_{t}+m_{\tilde{\chi}_{1}^{0}}$ because of very close topology with $t \bar{t}$. The exclusion weakens for higher LSP masses. The search for $\tilde{t}_{1} \rightarrow b \tilde{\chi}_{1}^{ \pm}$depends on the value of the chargino mass. For close-by stop and $\tilde{\chi}_{1}^{ \pm}$, two hard leptons are present in the final 
state and previously mentioned 2-leptons+jets analysis can be reused. If instead $\tilde{\chi}_{1}^{0}$ and $\tilde{\chi}_{1}^{ \pm}$are close-by, two hard $b$-jets will be present giving good sensitivity to direct sbottom analysis. Finally if $\tilde{\chi}_{1}^{0}$ and $\tilde{\chi}_{1}^{ \pm}$are far apart a mix of 1-lepton+4-jets and 2-leptons+jets is best. In the $\tilde{t}_{1} \rightarrow b \tilde{\chi}_{1}^{ \pm}$scenario, the stop could be excluded up to $600 \mathrm{GeV}$ but the message is less strong than for the right part of Fig. 32 since it depends on $\tilde{\chi}_{1}^{ \pm}$masses. Nevertheless in this case also stop masses are getting dangerously close to the upper bounds of the natural spectrum.

\subsubsection{Direct searches for EWKinos and sleptons}

Natural SUSY spectrum favors EWKinos close to the EW scale. Therefore they could well be the only sparticles accessible at LHC if colored ones are too heavy or decaying through intricate chains. The SUSY electroweak sector is characterized by low cross-sections and discoveries can only happen for lightest mass states $\left(\tilde{\chi}_{1}^{0}, \tilde{\chi}_{2}^{0}\right.$ and $\left.\tilde{\chi}_{1}^{ \pm}\right)$and leptonic final states. In the most 'natural' scenario, $\tilde{\chi}_{1}^{0}, \tilde{\chi}_{2}^{0}$ and $\tilde{\chi}_{1}^{ \pm}$are higgsino-like, i.e. almost mass degenerate. Therefore, for $\tilde{\chi}_{1}^{0}$ LSP models, when $\tilde{\chi}_{1}^{ \pm}$and $\tilde{\chi}_{2}^{0}$ decay to $\tilde{\chi}_{1}^{0}$, final states are composed of low energetic particles, hardly distinguishable from background. As a result, no limits exist on this scenario. Note that for $\tilde{G}$ LSP models, this constraint disappears since the mass difference between EWKinos and the $\tilde{G}$ is always significant, generating an interesting phase space, still poorly explored.

Another more favorable scenario consisting in binolike $\tilde{\chi}_{1}^{0}$ and wino-dominated $\tilde{\chi}_{2}^{0}$ and $\tilde{\chi}_{1}^{ \pm}$provides a viable solution when $\mu$ is not too high. In this case, the mass difference between $\tilde{\chi}_{2}^{0}$ and $\tilde{\chi}_{1}^{ \pm}$and the LSP increases, opening channels like $\tilde{\chi}_{2}^{0} \rightarrow Z^{0}\left(H^{0}\right) \tilde{\chi}_{1}^{0}$ and $\tilde{\chi}_{1}^{ \pm} \rightarrow W^{ \pm}\left(H^{ \pm}\right) \tilde{\chi}_{1}^{0}$ with on-shell $Z, H$ and $W$. The highest cross-section is coming from $\tilde{\chi}_{1}^{ \pm} \tilde{\chi}_{2}^{0} \rightarrow W(\rightarrow$ $l \nu) Z(\rightarrow l l) \tilde{\chi}_{1}^{0} \tilde{\chi}_{1}^{0}$. Assuming mass degeneracy between $\tilde{\chi}_{1}^{ \pm}$and $\tilde{\chi}_{2}^{0}$, a 3-lepton $+E_{T}^{\text {miss }}$ analysis excludes $M\left(\tilde{\chi}_{1}^{ \pm}, \tilde{\chi}_{2}^{0}\right)$ $<320 \mathrm{GeV}$ for LSP mass lower than $100 \mathrm{GeV}$, see Fig. 33 [114. Note that breaking the mass degeneracy between $\tilde{\chi}_{1}^{ \pm}$and $\tilde{\chi}_{2}^{0}$ will relax these upper bounds.

Searching for $\tilde{\chi}_{1}^{+} \tilde{\chi}_{1}^{-} \rightarrow W^{+}\left(\rightarrow l^{+} \nu\right) W^{-}\left(\rightarrow l^{-} \nu\right) \tilde{\chi}_{1}^{0} \tilde{\chi}_{1}^{0}$ in the 2-lepton $+E_{T}^{\text {miss }}$ is more challenging because $\sigma(W W$ $=10 \times \sigma\left(\tilde{\chi}_{1}^{+} \tilde{\chi}_{1}^{-}\right)$for $100 \mathrm{GeV}$ charginos. Vetoing jets and using similar discriminant variables as for direct sbottom searches, the $W W$ background can be reduced sufficiently to exclude few SUSY models [115. An interesting by-product of these searches is the possibility to exclude sleptons (selectron and smuon) at higher masses than at LEP, since both signal have the very same 2-lepton $+E_{T}^{m i s s}$ final state $\left(\tilde{l}^{+} \tilde{l}^{-} \rightarrow l^{+} l^{-} \tilde{\chi}_{1}^{0} \tilde{\chi}_{1}^{0}\right)$.
Finally $\tilde{\chi}_{1}^{+} \tilde{\chi}_{1}^{0} \rightarrow W^{+}\left(\rightarrow l^{+} \nu\right) \tilde{\chi}_{1}^{0}$ is not yet explored due to the very low cross-section and overwhelming inclusive $W$ cross-section. For the same reasons, the $\tilde{\chi}_{1}^{0} \tilde{\chi}_{1}^{0}$ production cannot be searched for, even in dedicated monojet analyses presented in Section 3.2.1. In conclusion, EWKino searches provide presently much weaker constraints on the natural SUSY scenario.

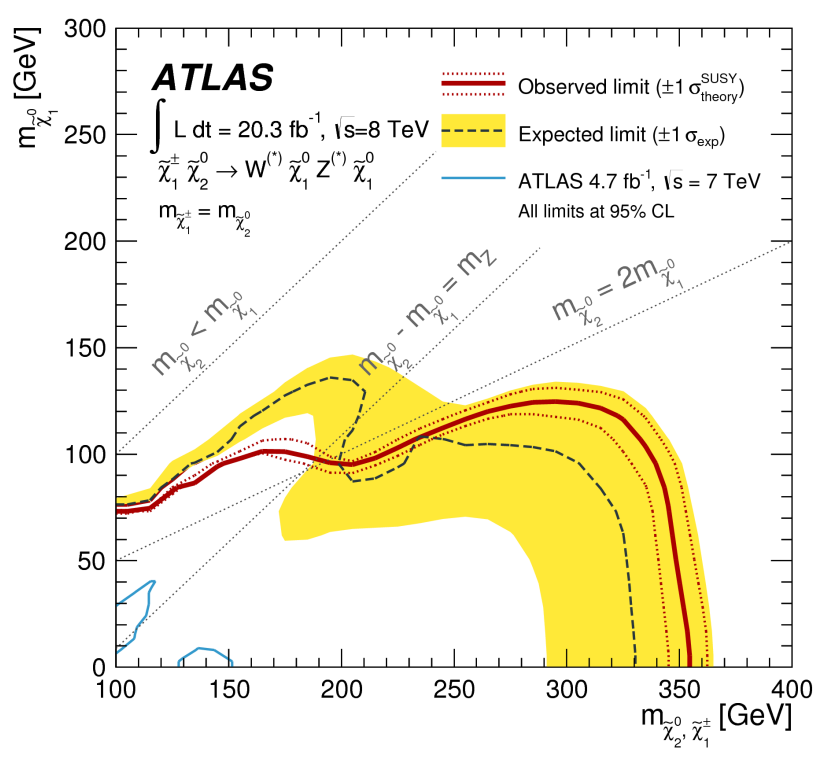

Fig. 33 Limits for associate EWKinos production as a function of the LSP mass [114.

\subsubsection{Status of R-Parity Conserved SUSY after LHC run $I$}

LHC have probed the uncharted heart of natural weak scale SUSY spectrum by direct searches. The limits are especially strong for gluinos and $3^{\text {rd }}$ generation squark in open spectra. Masses below $1 \mathrm{TeV}$ and 500-700 GeV are excluded, respectively. These constraints set plain vanilla MSSM on the grill and pushed it in corners of parameter space harder to access experimentally: com) pressed spectra and intricate decay chain (for strong SUSY), low cross-section processes (especially in the EW sector). To be more quantitative, a full scan of the most relevant 19-20 MSSM parameters (some assumptions are made on the other 105-19 parameters) was performed and the models surviving the LHC results examined. This study confirmed that spectrum evading the limits are typically the ones containing light stop and bottom with complex decay patterns [116, 117. 


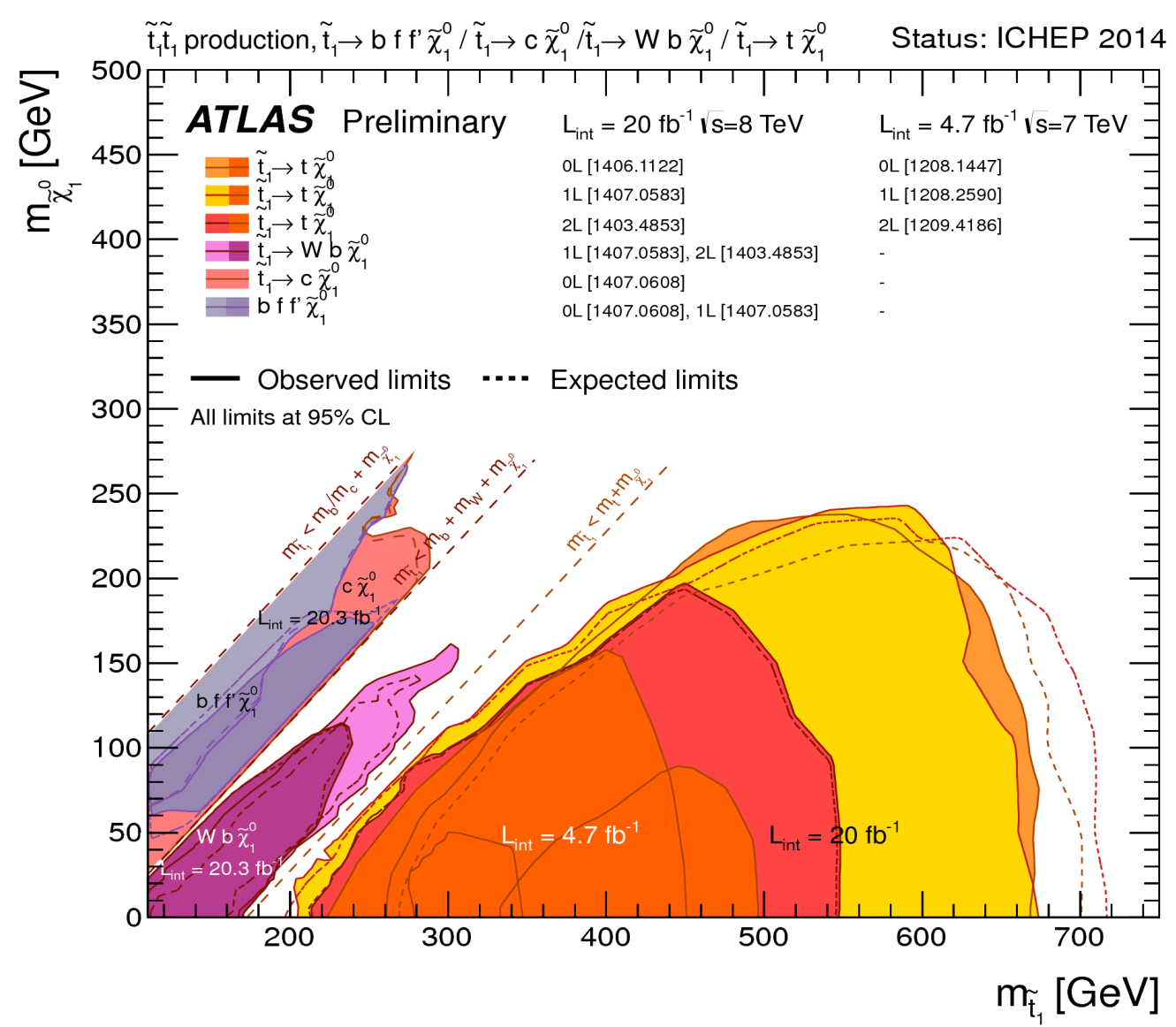

Fig. 32 Present limits in LSP-stop mass plane.

\subsubsection{Escape routes: Long-Lived particles, R-Parity Violation and others}

Beside the reasons given above, three possible escape routes could explain the null results in RPC SUSY searches. First, some particles of the SUSY spectrum can be metastable, i.e. they have non prompt decays within the inner detector giving non pointing $\gamma$ or $Z$, displaced vertices or disappearing tracks or even decay after the detector. Second, R-parity is violated i.e. one/several of the 48 Yukawa couplings in the superpotential 118 is/are non zero. Note that having all RPV parameters different from 0 is not possible because of the limits from proton lifetime. This generally gives striking signatures with lepton flavor violation $\left(\lambda_{i j k} \neq 0, \lambda_{i j k}^{\prime} \neq 0, i, j, k=1,2,3\right)$ or baryon number violation $\left(\lambda_{i j k}^{\prime \prime} \neq 0\right)$. The third possibility is a more complicated SUSY model, beyond the MSSM, relaxing the experimental constraints: for example a new singlet can be added, enlarging the EW sector with 2 more Higgs bosons and one more neutralino 119, or even SUSY could have no role in the hierarchy problem and only the LSP could be present at the TeV-scale, vestige of a very high mass scale spectrum [120. As the phase space is huge and less well-defined than in the RPC case, only some illustrative examples are discussed below.

As no metastable particles are present in the Standard Model their searches are generally background free. Their discovery in-turn requires a deep understanding of the detector performance, which represent the only background. In SUSY, non-prompt particle decay can be caused by $i$ ) very weak R-Parity violation, i.e. one of the Yukawa coupling $\lambda, \lambda^{\prime}$ or $\lambda^{\prime \prime} \leq \mathrm{O}\left(10^{-5}\right)$, ii) very low mass difference between a SUSY particle and the LSP in RPC model or iii) very weak coupling to the gravitino in GMSB models. AMSB provides a well motivated case for $i i)$ where $\tilde{\chi}_{1}^{ \pm}$and $\tilde{\chi}_{1}^{0}$ are almost degenerate and $M\left(\tilde{\chi}_{1}^{ \pm}\right)-M\left(\tilde{\chi}_{1}^{0}\right) \geq 140 \mathrm{MeV}$. The chargino is therefore metastable and decays after few tens of centimeters to undetectable particles, a soft pion and the LSP. This will cause the chargino track to 'disappear'. When produced directly $\left(\tilde{\chi}_{1}^{+} \tilde{\chi}_{1}^{-}, \tilde{\chi}_{1}^{ \pm} \tilde{\chi}_{1}^{0}\right)$ with an additional jet from initial state radiation to trigger 
the event, one (or two) tracks may have no/few associated hits in the outer region of the tracking system. The continuous tracking of the outer part of the ATLAS inner detector, the straw tube transition radiation (TRT), gives sensitivity to this signature and removes the background. With the additional requirement of an high energetic isolated track, regions beyond the LEP limits can be excluded in the lifetime-mass plane of the chargino, as shown in Fig. 34 121. Although originally motivated by AMSB, this result is largely model independent and is also predicted by unnatural SUSY [120].

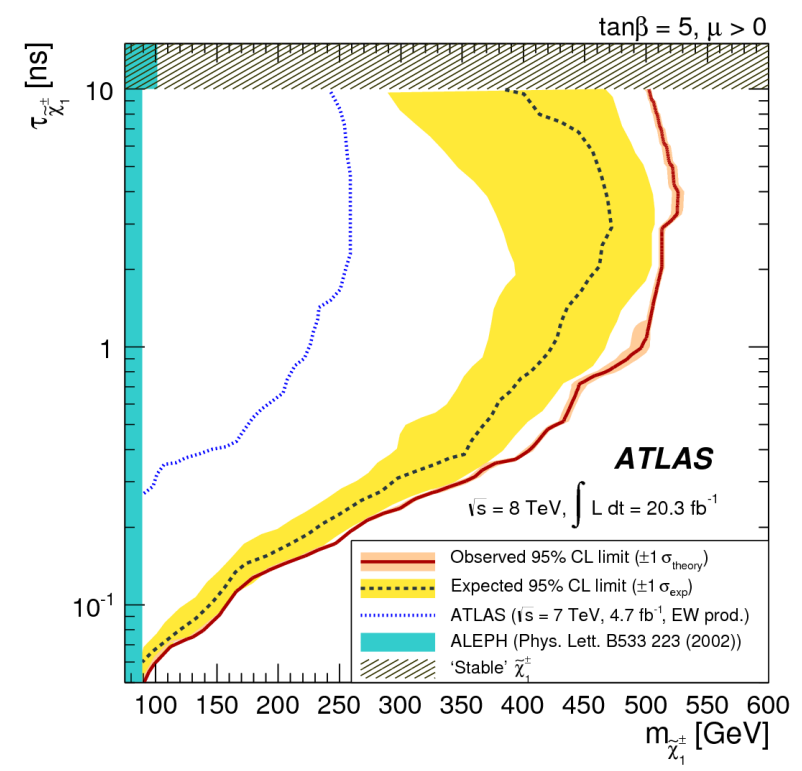

Fig. 34 Limits in the lifetime-mass plane for a metastable chargino 121 .

If gluino and LSP are almost mass degenerate, its lifetime could be long enough for him to hadronize in $R$ hadrons $(\tilde{g} q \bar{q}, \tilde{g} q q q)$ or $R$-gluino balls $(\tilde{g} g)$. A fraction of these slow moving particles may come to rest within the detector volume and only decay later as $\tilde{g} \rightarrow q \bar{q} \tilde{\chi}_{1}^{0}, g \tilde{\chi}_{1}^{0}$. If this happens in the calorimeter, the signature will be high energetic jet(s) in absence of collisions. In that case, the background comes from the calorimeter noise burst, cosmic ray with high energy deposit or beam halo - the leading background. Gluinos below $850 \mathrm{GeV}$ are excluded for a gluino lifetime between $10 \mu \mathrm{s}$ and 15 minutes [122].

Sizable R-Parity violation in one of the Yukawa coupling $\left(\sim 10^{-4,-2}\right)$ can easily give four or more leptons because of the LSP decay (e.g. $\lambda_{121} \neq 0$ ) or $2 \times 3$-jet resonances $\left(\lambda_{i j k}^{\prime \prime} \neq 0\right)$. In the former case, assuming $\tilde{g} \tilde{g} \rightarrow q q \tilde{\chi}_{1}^{0}\left(\rightarrow l^{+} l^{-} \nu\right) q q \tilde{\chi}_{1}^{0}\left(\rightarrow l^{+} l^{-} \nu\right)$ allows to exclude gluino below $1.4 \mathrm{TeV} \quad 123$ while in the latter case gluino just below $1 \mathrm{TeV}$ are excluded by asking 6-jets to be reconstructed with a minimum energy as expected from $\tilde{g} \tilde{g} \rightarrow \tilde{q}(\rightarrow q q) q \tilde{q}(\rightarrow q q) q[124]$.

In all these models it is generally true that the gluino mass is excluded for masses below $1 \mathrm{TeV}$.

\subsection{Searches for other natural theories at LHC}

As discussed in Section 2.5, alternatives to SUSY exist to solve the hierarchy problem. They generally have distinct features compared to SUSY signatures: higher cross-section, moderate or null $E_{T}^{\text {miss }}$, high mass resonances decaying to very energetic calorimeter objects (electrons, photon and jets) or boosted top, $W$ or $Z$. In the latter case, very collimated objects are produced and reconstructed into one single "fat" jet. Using the high granular ATLAS and CMS detectors, dedicated algorithms were developed to look for substructure in very high energetic jets and to separate the initial objects. These methods greatly improve the reconstructed top, $W$ or $Z$ mass resolution, increasing the sensitivity to new physics.

\subsubsection{Large Extra Dimensions}

The most striking possibility is that gravity is strong close to the EW scale. Assuming its lines of force propagate in $4+\delta$ large flat extra spatial dimensions (the "bulk"), gravity will be "artificially" weak in our four dimensional rigid brane, where SM particles are confined. In this model, called ADD [125], the hierarchy problem needs to be rewritten. A $\delta$-dimension fundamental Planck mass, $M_{D}$, can be computed as a function of the compactification radius $R$ of the extra dimensions on a $\delta$-dimensional torus or a sphere as:

$M_{D}=\left[\frac{M_{P l}^{2}}{R^{\delta}}\right]^{-\frac{1}{2+d}}$

If $M_{D} \sim 1 \mathrm{TeV}$, the hierarchy problem is solved and as $\hbar c=2 \times 10^{-14} \mathrm{GeV} . \mathrm{cm}$,

$R=2 \times 10^{-17+32 / \delta} \mathrm{cm}$

Only $\delta=1$ is excluded experimentally. In the bulk, gravitational interaction are mediated by massless graviton and Kaluza-Klein (KK) graviton towers $G^{(k)}$ are predicted in the $4 \mathrm{D}$ brane with masses:

$m_{k}^{2}=m_{0}^{2}+k^{2} / R^{2}, k=0,1,2,3,4, \ldots$

For large $R$, the KK states are almost continuous which compensate the small graviton coupling $\left(\sim 1 / M_{P l}\right)$. Three of the most spectacular signatures expected at LHC are now discussed. 
First, the direct production of KK gravitons via the processes $q \bar{q} \rightarrow g G, q g \rightarrow q G, g g \rightarrow g G$ could provide a monojet signature as the graviton escape detection [126. In this scenario a larger tail is expected in the $E_{T}^{\text {miss }}$ distribution, see Fig. 35. compare to the dominant background $Z \rightarrow \nu \nu$ plus one jet emitted in the initial state. The search is limited by the statistics in the $Z \rightarrow \nu \nu$ control region where two leptons are required on top of the jet and $E_{T}^{\text {miss }}$ kinematic cuts. Nonetheless a huge range of models can be excluded with respect to LEP and Tevatron as shown in Fig. 36 [127.

Another interesting signature exists when $s$-channel KK gravitons exchange takes place and KK gravitons decay to dibosons, dileptons and/or dijets, causing large invariant masses. One of the most promising signature is the search for diphoton resonance since it is possible to reduce the $\gamma$-jet and jet-jet background below the irreducible $\gamma \gamma$ background from SM (Section 2.4.1). The ADD signal will appear as a wide resonance over the background, see Fig. 37 [128. This channel provides similar limits as the monojet analyses for $\delta>2$.

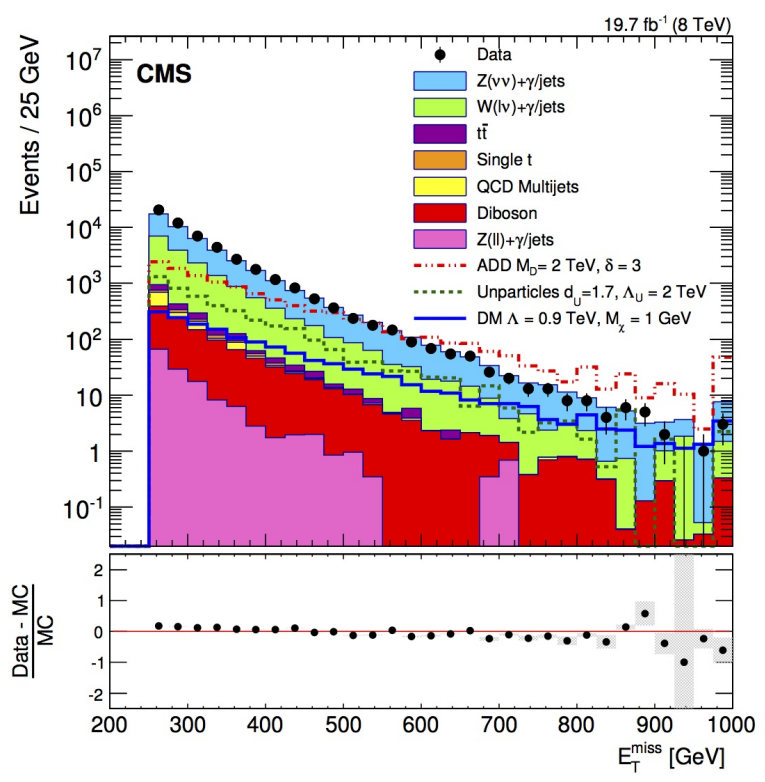

Fig. 35 ADD model searches at LHC: $E_{T}^{m i s s}$ distribution in Monojet analysis [127.

Finally since $\sqrt{s}>M_{D} \sim 1 \mathrm{TeV}$, gravity is enhanced in the $4+\delta$ space and microscopic black holes could be produced at LHC. They will then evaporate through Hawkings radiation in a high multiplicity $(N)$ of particles, see Fig. 38. Here the background is dominated by QCD and estimated assuming a common shape for $H_{T}$ regardless of $N$. Large uncertainties exist on these Black Hole (BH) production models due to our ig-

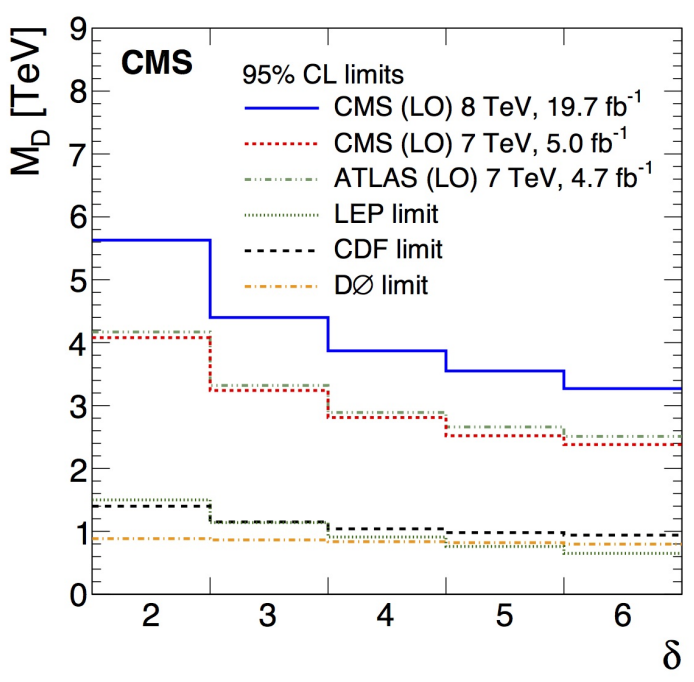

Fig. 36 Exclusion of ADD Models with Monojet analysis for different $\delta$-dimension [127].

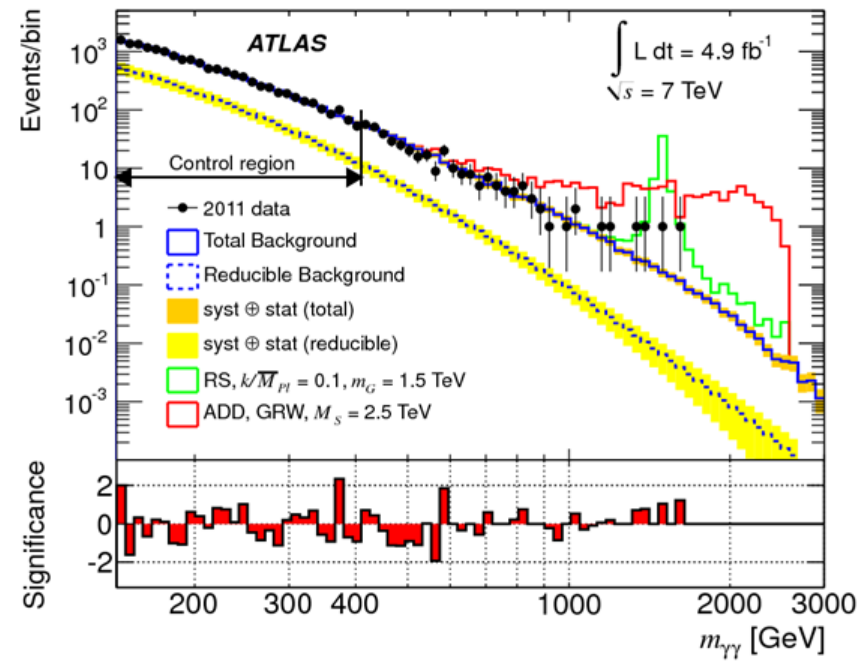

Fig. 37 ADD model searches at LHC: diphoton resonance 128 .

norance of quantum gravity. Assuming (semi-)classical approximation are valid for $M_{B H}>M_{D}$, quantum black holes with masses below 4.3-6.2 TeV are excluded [129]. Other signatures can be used like 2 same sign muons [130] or even lepton+jets signature [131].

\subsubsection{Warped Extra Dimensions}

The hierarchy problem can also be solved by considering only one extremely small new compact dimension with a warped geometry of curvature $k \approx M_{P l}$ where only gravity propagates 132 . This set-up, called minimal Randall-Sundrum (RS), is composed of a 5 di- 


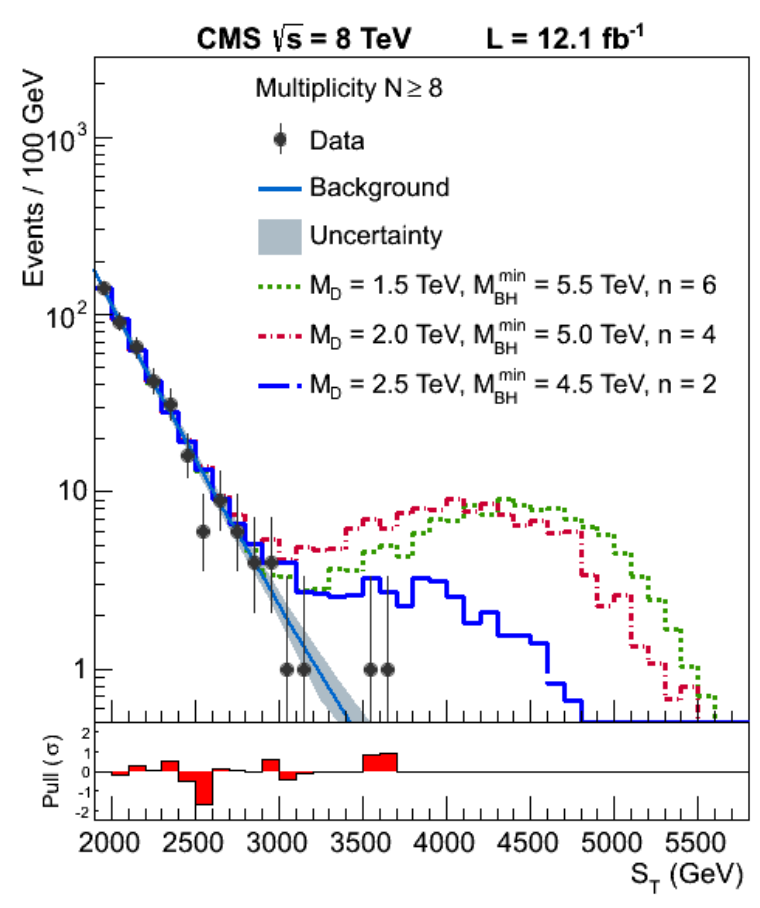

Fig. 38 ADD model searches at LHC: total transverse energy for $N \geq 8$ objects $\left(e, \mu, \gamma\right.$, jets). $S_{T}$ means $H_{T}$ here and $n=\delta$ [129].

mensional bulk with one compactified dimension, and two 4D branes, called SM and gravity branes. In these conditions the Planck scale is red-shifted for SM brane observers and becomes $M_{D}=M_{P l} e^{-k \pi R}$. For $k R \sim 12$, i.e. $R=10^{-32} \mathrm{~cm}, M_{D} \sim 1 \mathrm{TeV}$ which solve the hierarchy problem.

Experimental consequences are very different from the ADD case: KK graviton masses are not regularly spaced but given by $m_{n}=x_{n} k e^{-k \pi R}$ where $x_{n}$ are the roots of Bessel functions. Only the first excitation, $G^{(1)}$, with a narrow width $k / M_{P l}<1$, is generally accessible at LHC. Its coupling to SM particles is proportional to $1 /\left(M_{P l} e^{-k \pi R}\right)$ and therefore much stronger than for ADD model. As a consequence the main experimental evidence is a narrow peak in the diboson (Fig. 37) or dilepton (Fig. 39 invariant mass. For $k / M_{P l}=0.02$, $G^{(1)}$ masses below $2.7 \mathrm{TeV}$ are excluded [133].

Since solving the hierarchy problem requires only the Higgs to be close to the SM brane, the minimal RS can be modified by allowing SM fields to propagate also in the bulk [134]. This has the extra advantage to explain the SM Yukawa coupling hierarchies by the position of the SM fields in the bulk. All SM fields create KK towers which are constrained to have below than 2-3 TeV masses for the first excitation [135. A particularly interesting search comes from the KK gluon $\left(g_{K K}\right)$ decaying to $t \bar{t}$ which provides an enhancement at high mass of $t \bar{t}$ invariant mass spectrum, as shown in Fig. 40 for the all hadronic channel [136]. Combining all sensitive $t \bar{t}$ decay channels, $g_{K K}$ masses are excluded below $2.5 \mathrm{TeV}$ 137] getting close to the upper part of the allowed region. In these RS-bulk models the cross-section of $g g \rightarrow G^{(1)} \rightarrow W W(Z Z)$ is driving the $G^{(1)}$ hunt motivating a search for $Z Z$ or $W W$ resonances [138,139. However, presently, no mass limit beyond $500 \mathrm{GeV}$ can be put on $G^{(1)}$ when $0.04<k / M_{P l}<0.1$.

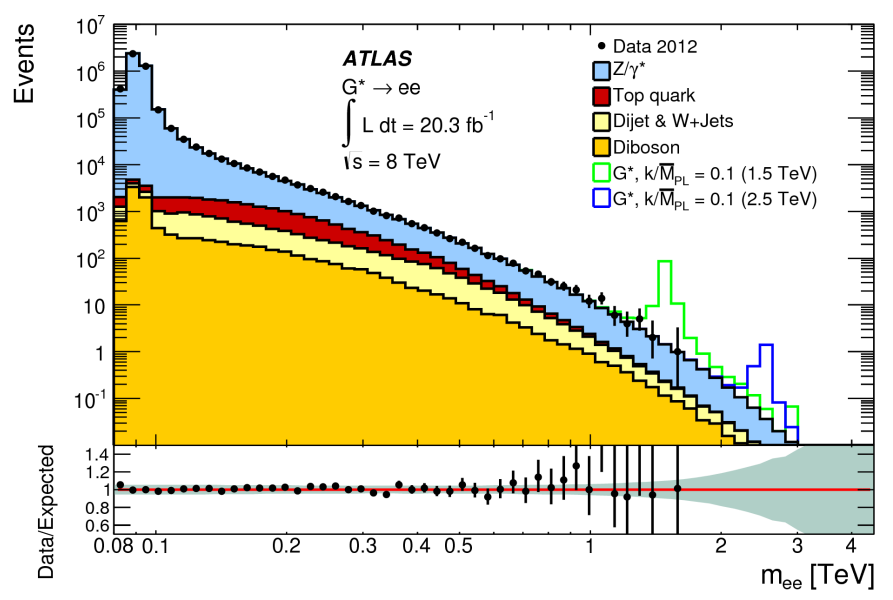

Fig. 39 Warped extra dimension searches at LHC: dielectron resonance [133.

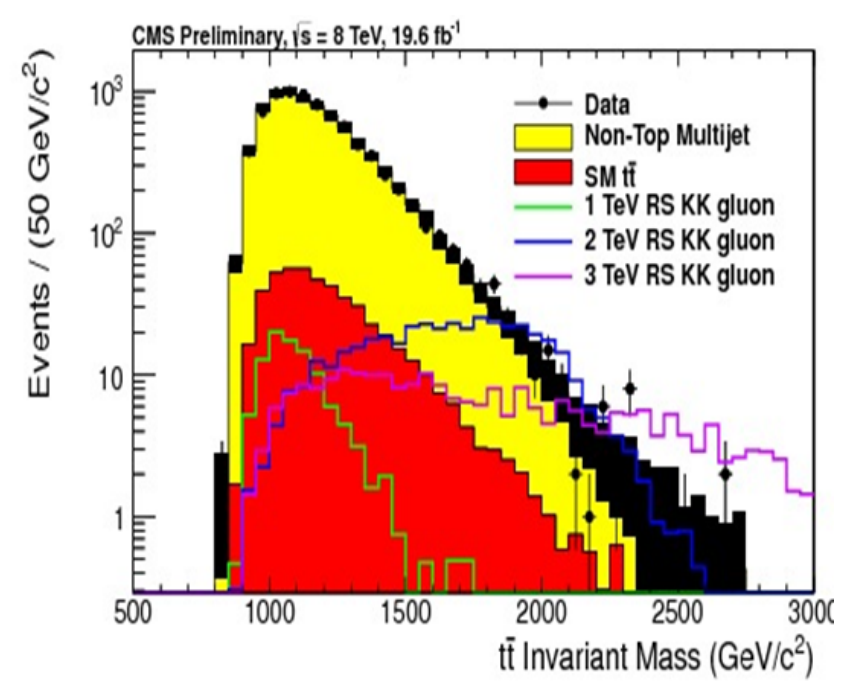

Fig. 40 Warped extra dimension searches at LHC: $t \bar{t}$ resonance [136]. 


\subsubsection{Composite Higgs models}

All experimental results indicate today that the new discovered particle with a mass of $125 \mathrm{GeV}$ is the SM Higgs boson. However it is not excluded that the Higgs boson is a composite particle. This would then be interpreted as the first manifestation of a new strong sector that should appear at a scale $f \sim \mathrm{O}(\mathrm{TeV})$. Note that the Higgs couplings will then be modified by $\sim$ $\mathrm{O}\left(v^{2} / f^{2}\right)$, i.e. remain quite close to the $\mathrm{SM}$ values and compatible with the present measurements. If the Higgs is composite, it could play a similar role as the neutral pion for the strong force, a pseudo Goldstone boson of a spontaneously broken new global symmetry. This could explain also why it is much lighter than the other (unobserved) resonances.

Many models have been constructed upon this generic idea, from technicolor to little Higgs models. As of today, composite Higgs models [140] are probably the less constrained ones. These models also present the advantage to be related by holography to weakly-coupled models containing a warped extra dimension, presented just before. The advantage is that perturbative computations can be performed in these extra dimension models and later be used to derive the properties of the expected composite states.

In composite Higgs models, the Higgs mass is typically around $0.2 \mathrm{TeV}$ or higher but $125 \mathrm{GeV}$ could still be accommodate. The hierarchy problem is solved by the finite size of the Higgs, which screens the contributions to its mass from $\mathrm{O}(\mathrm{TeV})$ new particles, a similar mechanism as for SUSY. These new particles are vectorlike top partners and should be light (around $0.7 \mathrm{TeV}$ ), or $Z^{\prime}$ and $W^{\prime}$ which should be in the $1-3 \mathrm{TeV}$ mass range. As in SUSY models, the discovery of these top partners $(T)$ is the most pressing issue. To evade the EW precision fit constraint, $T$ could have the form of an electroweak singlet of charge $2 / 3$ and searched for via direct production and the subsequent decay to $t W$, $b W, t Z, t H$. These decays generate multi- $W$, i.e 1,2 and/or 3-lepton+jets final states. When all these channels are combined, it is possible to exclude $T_{2 / 3}$ with masses below $687 \mathrm{GeV}$ as shown in Fig. 41 [141. Another possibility is $T_{5 / 3} \rightarrow t W$ and similar limits are obtained with a 2 lepton same sign analysis [142].

\subsubsection{Preliminary conclusions on searches for Natural theories}

Since 40 years many BSM theories were developed to solve the hierarchy problem with new physics strongly or weakly interacting with the Higgs. This is today the most outstanding problem of the SM, getting even

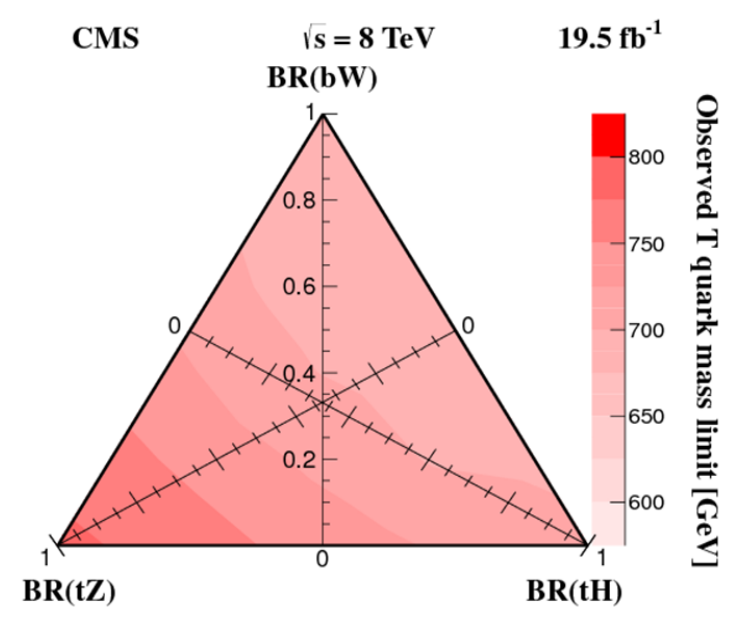

Fig. 41 Status of $T_{2 / 3}$ search [141].

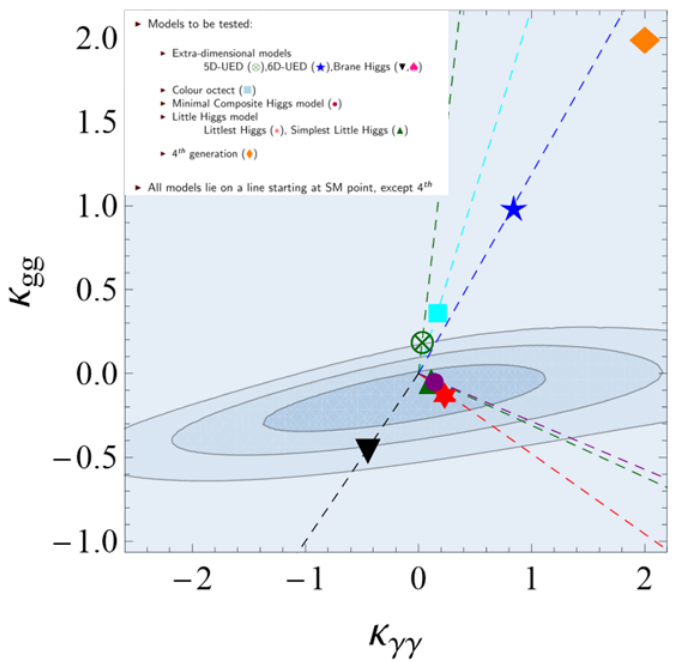

Fig. 42 Illustration of the $4^{\text {th }}$ generation quark model (diamond on the top right) exclusion by Higgs couplings measurements (shaded area) [143.

stronger with the discovery of a SM Higgs-like particle. Unexpectedly, no sign of new physics have been observed with the LHC run I data and most of these BSM theories are now seriously cornered. The new predicted particles are now generally excluded close or above the 1 TeV scale. LHC seems to disfavor a 'natural' scenario, even if all results have not been obtained and some holes still exist in analyses. The complete analysis of the next LHC run at $13-14 \mathrm{TeV}$ will enable to make a more definitive statement. 


\subsection{Other Beyond Standard Model searches at LHC}

As mentioned in Section 2.5 there are many other possible SM extensions that do not solve the hierarchy problem but addresses other conceptual problems. I just highlight here two very important searches. The first one is probing the quark compositeness by looking for a resonance in a dijet invariant mass $m_{j j}$ spectrum [144] and measuring the relative proportion of central jets per $m_{j j}$ bins. A bump in the $m_{j j}$ distribution or an increase of central jets at high $m_{j j}$ could reveal a new substructure [145, like the gold-foil Rutherford experiment revealed the atomic nucleus. In both cases, null results are obtained and excited quarks below $\sim 4 \mathrm{TeV}$ are excluded. The second search for the presence of a fourth generation of quarks which can be directly excluded by the Higgs coupling measurements. As discussed in Section 2.4.1. the Higgs production and decay in $H \rightarrow \gamma \gamma$ channel occurs almost entirely by triangle heavy fermion loops. Therefore an enhancement is expected, in both cases, in presence of $4^{\text {th }}$ generation quarks. As shown in Fig. 42, these models are already excluded 143. Results not mentionned here can be can be found on the experiment websites 146, 147.

Since this lecture concentrates on ATLAS and CMS results, it worth to remind that precision measurements and rare decay searches are also very sensitive to the presence of new physics far below the TeV scale: not as a direct evidence but as deviations from SM expectations that could be explained by "virtual" effects including new physics. Most powerful probes are provided by the proton decay, lepton flavor violation, FCNC in the quark sector, electric dipole momentum [148, 149.

\subsection{Impact of LHC results on Dark Matter searches}

Dark matter (DM) is required to form the observed large scale structures of the universe and is one of most serious challenge for the Standard Model of Particle Physics. The five requirements for a particle to be a dark matter candidate $\chi$ can be spell out as: gravitationally interacting at cosmological and astrophysical scales (the only actual proof that DM exists), not short lived, not hot, not baryonic and giving at most the right thermal relic density as measured by CMB experiments, $\Omega h^{2}=0.120 \pm 0.003$, where $h$ is the Hubble constant. The second condition removes all SM particles apart from the neutrinos and the fermions $(e, u, d)$. The third condition rejects the neutrinos and the fourth one the rest of the fermions. So SM does not provide any viable DM candidate while "natural" BSM theories does, as shown in Fig. 43. Assuming that dark matter is explained by only one particle with mass $m_{\mathrm{DM}}$ and the relevant gauge coupling constant $g_{\mathrm{DM}}$, then $\Omega_{\chi} \propto m_{\mathrm{DM}}^{2} / g_{\mathrm{DM}}^{4}$. With this in mind, three categories can be formed: $i$ ) the WIMP sector where $m_{\mathrm{DM}} \approx \Lambda_{\mathrm{EW}}$ and $\left.g_{\mathrm{DM}}=g_{\mathrm{EW}}=2\left(\sqrt{2} G_{F}\right)^{1 / 2} m_{W} \approx 0.65, i i\right)$ the hidden sector, gathering SuperWIMP and axions ${ }^{5}$. where $m_{\mathrm{DM}} \leq \Lambda_{\mathrm{EW}}$ and $g_{\mathrm{DM}} \ll g_{\mathrm{EW}}$ and $\left.i i i\right)$ the undetectable sector with fuzzy dark matter, where the interaction is purely gravitational. Among all candidates, WIMP particles are still the most popular since they are motivated by the resolution of the hierarchy problem, a completely uncorrelated reason (the so-called "WIMP miracle").

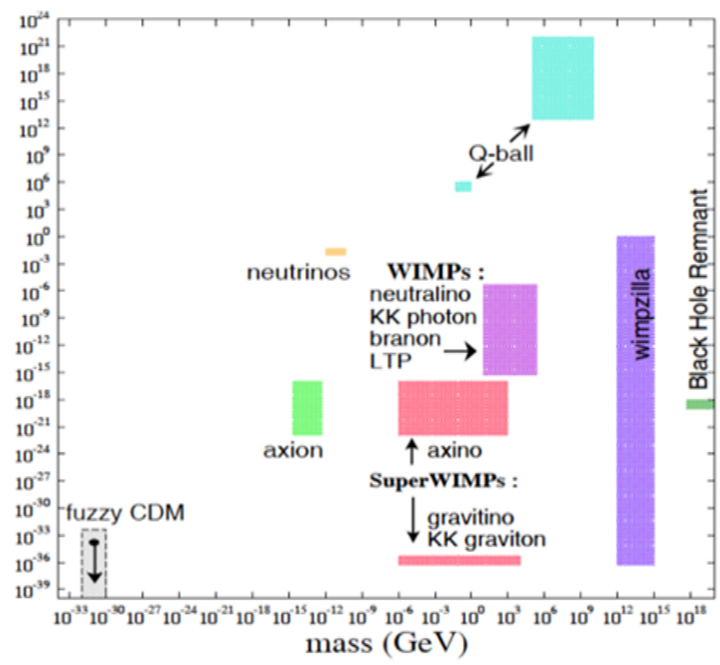

Fig. 43 DM candidate particles shown in the plane $\chi$ nucleon cross-section (pb) versus $\chi$ mass 150 .

Since a lot of information is already available in other lectures of this school 151 and in excellent reviews [152,87, I'll only discuss the LHC input to the DM search. By analogy with the weak interaction described by Fermi theory, DM could be produced at LHC via $q \bar{q}, q g, g g \rightarrow X \rightarrow \chi \bar{\chi}$ and could be observed in a monojet analysis (the jet is an initial state radiation) see Fig. 35 . The mediator $X$ of mass $M$ could be scalar, vector or axial-vector, and interact with quark and WIMP with coupling factors $g_{q, g}$ and $g_{\chi}$. The contact interaction scale is then defined as $\Lambda=M / \sqrt{g_{q, g} g_{\chi}}$. This approach allows the conversion into DM-nucleon cross-section limits for a given $\chi$ mass, directly comparable with dedicated DM searches [153,154]. For vectorlike mediator, Fig. 44 shows that LHC could exclude low mass WIMP where direct searches have no sensitivity because of undetectable energy recoil of the nucleon. For axial-vector mediator or DM-gluon coupling

${ }^{5}$ The name of the hypothetical particle resolving the strong CP problem. 


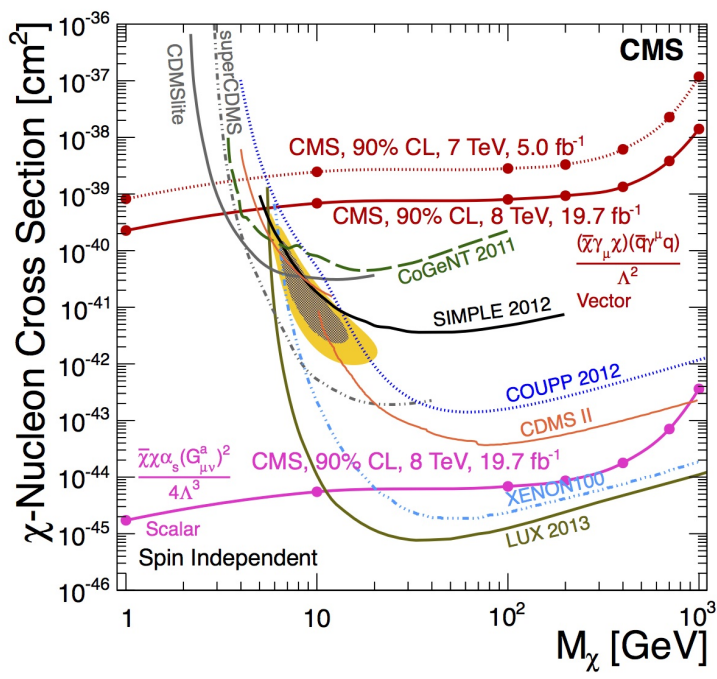

Fig. 44 Exclusion curves obtained in the same plane by LHC and direct DM search experiments, assuming a vector-like mediator for the $\chi$-nucleon interaction 127 .

with scalar or vector mediators, LHC results exceed all present limits [127.

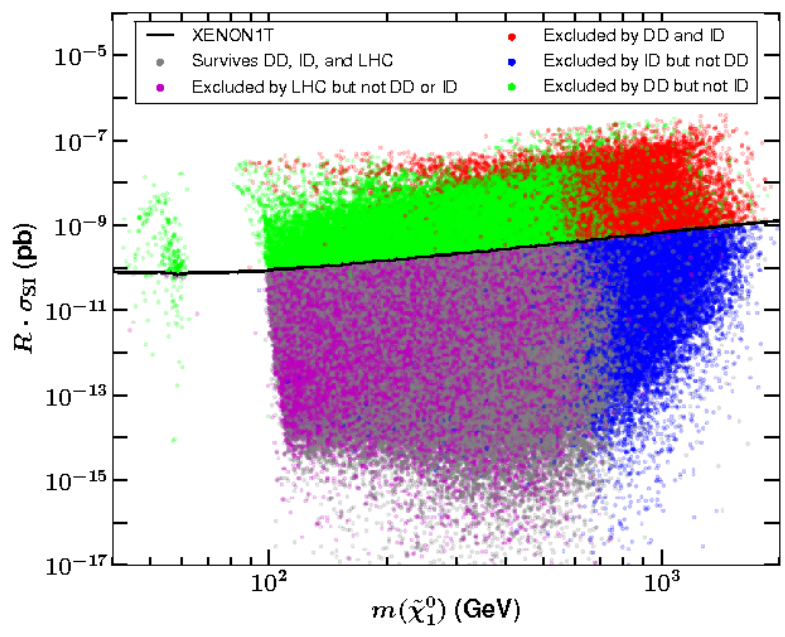

Fig. 45 MSSM models and experimental constraints in the $\chi$-nucleon cross-section- $m_{\tilde{\chi}_{1}^{0}}$ plane [155].

The leading WIMP candidate is (still) $\tilde{\chi}_{1}^{0}$, but it can not be probed by monojet analysis because of the too low cross-section, $\sigma=\mathrm{O}(1)$ fb for $m_{\tilde{\chi}_{1}^{0}}=100 \mathrm{GeV}$. In direct SUSY searches $m_{\tilde{\chi}_{1}^{0}}$ is generally not directly accessible, but many models predicting $m_{\tilde{\chi}_{1}^{0}} \simeq 500$ $600 \mathrm{GeV}$ are currently excluded (Section 3.1). It is therefore interesting to scan a subset of MSSM models, satisfying the present experimental LHC constraints from

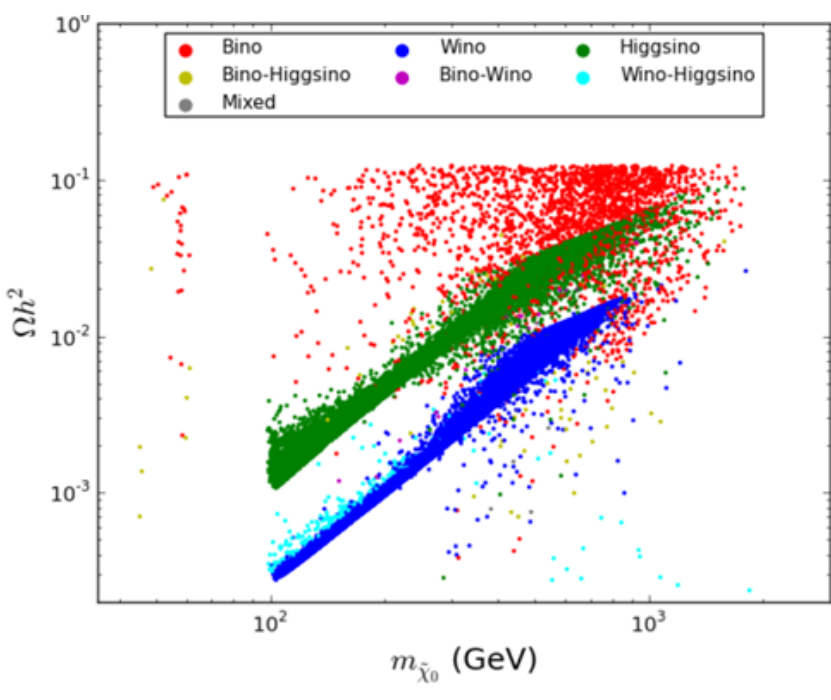

Fig. 46 LSP composition of surviving pMSSM models in the thermal relic density- $m_{\tilde{\chi}_{1}^{0}}$ plane 155 .

direct SUSY searches and Higgs mass, and see what flavor and mass range of $\tilde{\chi}_{1}^{0}$ survives 155 . Constraints from direct and indirect DM non-LHC searches can also be included, as mentioned above and the complementarity of the different approaches appears clearly in Fig. 45. The surviving models are shown in Fig. 46. Interestingly a huge quantity of models is still alive today and the only models which saturate the thermal relic density have bino-like LSP. Note also that almost all surviving models will be reachable by experiments in a near future. To conclude, it is worth to mention that the $Z H(\rightarrow \chi \chi)$ searches, not included in this study, are also excellent probes for $m_{\mathrm{DM}}<m_{H} / 2$, and covers $\sigma_{\chi-\text { Nucl }} \sim 10^{-7}-10^{-11} \mathrm{pb}$ depending on the mediator properties [156].

\subsection{Neutrinos and Baryogenesis}

Because neutrinos only interact with matter by exchanging very massive $W$ and $Z$, LHC experiments can not explore the neutrino sector. Instead dedicated experiments are built near nuclear plants or in deep underground mines where atmospheric, solar or intense neutrino beams from a particle physics center could be studied. Last 15 years saw many new results: discovery of neutrino oscillation [157, neutrino-tau [24] and recently measurement of third PMNS mixing angle $\theta_{13}$ [158].

Before going further, let's recall the peculiar position of the neutrinos in the SM: $i$ ) the only neutral fermions, $i i$ ) the only particles with unknown masses, present upper limits from direct measurement gives $m<$ 
$2 \mathrm{eV}$ [159], iii) the only fermions with no right-handed partners, $i v$ ) the only sector where original SM setting, $m_{\nu}=0$ and no mixing, was incorrect, $v$ ) the only fermions giving a cosmic background $(\mathrm{C} \nu \mathrm{B})$, expected at $\mathrm{T}=1.95 \mathrm{~K} \sim 0.17 \mathrm{meV}$. On top of this singular situation in the SM four fundamental questions remain unanswered: Are neutrinos Majorana or Dirac fermions? What is the absolute mass scale of neutrinos and what is their hierarchy (normal or inverted)? What are the precise values of PMNS matrix elements, and especially the $\mathrm{CP}$ violation phase? And finally are there "sterile" neutrinos, i.e. neutrino interacting only with the Higgs and other lepton doublets but not $W$ or $Z$ ?

While waiting for experimental answers, the question of the very low neutrino masses compared to other fermions triggered very interesting theoretical developments. The preferred explanation relies currently on the so-called "see-saw" mechanism pioneered at the end of the 70's [160,161,162,163. The plain vanilla scenario assumes Majorana neutrinos $(\bar{\nu}=\nu)$ and predict 3 new particles, the right-handed sterile neutrino singlets $N_{R i}$. Since $N_{R}$ s do not interact with gauge fields, very high masses $M \gg \Lambda_{\mathrm{EW}}$ are possible. Meanwhile left-handed neutrinos need to be massless to conserve the gauge invariance. With left and right-handed neutrinos, the Higgs field generates neutrino Dirac masses $m_{D, i}=v h_{i}$ as for quarks and other leptons. The neutrino mass matrix can therefore be written $\left(\begin{array}{cc}0 & m_{D} \\ m_{D} & M\end{array}\right)$ with two neutrino eigenvalues $m_{\nu}=m_{D}^{2} / M$ and $m_{N}=M$. Assuming very low left-handed neutrino masses $m_{\nu} \sim 0.1 \mathrm{eV}$ and $h_{i} \sim 1$ gives very heavy right-handed neutrinos $m_{N}=10^{14-15} \mathrm{GeV}$. Under these assumptions, a very interesting byproduct can be derived. It has been known since the end of the 70's that the presence of GUTscale mass particles could be a natural way to generate matter-anti matter asymmetry 164. Later, it was realized that the very high mass right-handed neutrinos could play this role, assuming the reheat temperature is higher than $N_{R}$ masses [165], see [166] for a recent review. Indeed in this case the three Sakharov conditions [167] are satisfied:

- For $T<m_{N}, N_{R}$ will be out of equilibrium (Sakharov 3)

$-N_{R} \rightarrow l^{+} H$ and $N_{R} \rightarrow l^{-} H$ decays violate the leptonic number conservation. $\mathrm{CP}$ violation in the lepton sector is expected from the PMNS matrix as a single complex phase like for the quark sector in the CKM matrix (Sakharov 2).

- The lepton asymmetry can be converted to baryon asymmetry by non perturbative SM processes called sphaleron (Sakharov 1).

This scenario is particularly popular since: $i$ ) the EW baryogenesis is not possible in the SM with the recently measured Higgs mass (Section 2.4.3, ii) CP violation in the quark sector is not large enough to generate the observed baryon asymmetry [168] and iii) it turns natural if weak-scale SUSY is realized since $N_{R}$ will have a superpartner with similar mass. Note also that many other scenarios exist as the see-saw mechanism is viable down to very low values of $h_{i}$. For example for $h_{i}=10^{-6,-8}$, the lightest subGeV $N_{R}$ will be long-lived, a good dark-matter candidate and could explain baryogenesis [169.

\subsection{The future of experimental Particle Physics}

Neutrino and collider experiments have paved the way of particle physics in the last 50 years. Prospects in the next decades are now briefly discussed, focussing on new colliders addressing the energy frontier. A more thorough review can be found in [170]. Collider experiments must address the two central questions of particle physics: detail understanding of the recently discovered scalar, i.e. precise measurement of all Higgs couplings, and thorough search for BSM particles in the TeV scale range. The next step is obviously the LHC restart at $\sqrt{s}=13-14 \mathrm{TeV}$ in 2015. By 2018 (2022), 50 (300) $\mathrm{fb}^{-1}$ of data should be collected. New particles with masses augmented by a factor 1.5-2 compared to present limits should be accessible. By 2022, a 5$15 \%$ precision on all Higgs couplings, except $c$-quark Yukawa coupling, should be at hand [171]. Beside this point, three projects are in competition:

- A High Luminosity LHC (HL-LHC) in 2024-2030 whose aim is to obtain $3000 \mathrm{fb}^{-1}$ of data at $\sqrt{s}=$ $14 \mathrm{TeV}$. Here also substantial improvements could be obtained in the Higgs sector as well as a first measurement of Higgs self-coupling.

- A linear $\mathrm{e}^{+}-\mathrm{e}^{-}$collider (ILC) that could start in Japan before 2030 with a $\sqrt{s}=250 \mathrm{GeV}$ and serve as a Higgs Factory. Full program includes an increase at 500 and $1000 \mathrm{GeV}$. Extra improvements on Higgs coupling precision by factors 2 to 10 , depending on the particle type, could be achieved.

- A circular $\mathrm{e}^{+}-\mathrm{e}^{-}$collider (LEP3) with $\sqrt{s}=240$ $\mathrm{GeV}$ could also be a very powerful Higgs Factory with similar or better sensitivity. The idea is to dismount the LHC, install LEP-like set-up in place, and run from 2024 on.

Beyond 2030, higher energy machines (VLHC, CLIC and TLEP) could be the continuation of the three previous projects with $\sqrt{s}=26-100,0.5-3.0$, and $0.24-0.35$ $\mathrm{TeV}$ respectively. These programs require new tunnels in the CERN area: $80 \mathrm{~km}$ for VLHC and TLEP and $13-48 \mathrm{~km}$ for CLIC. 


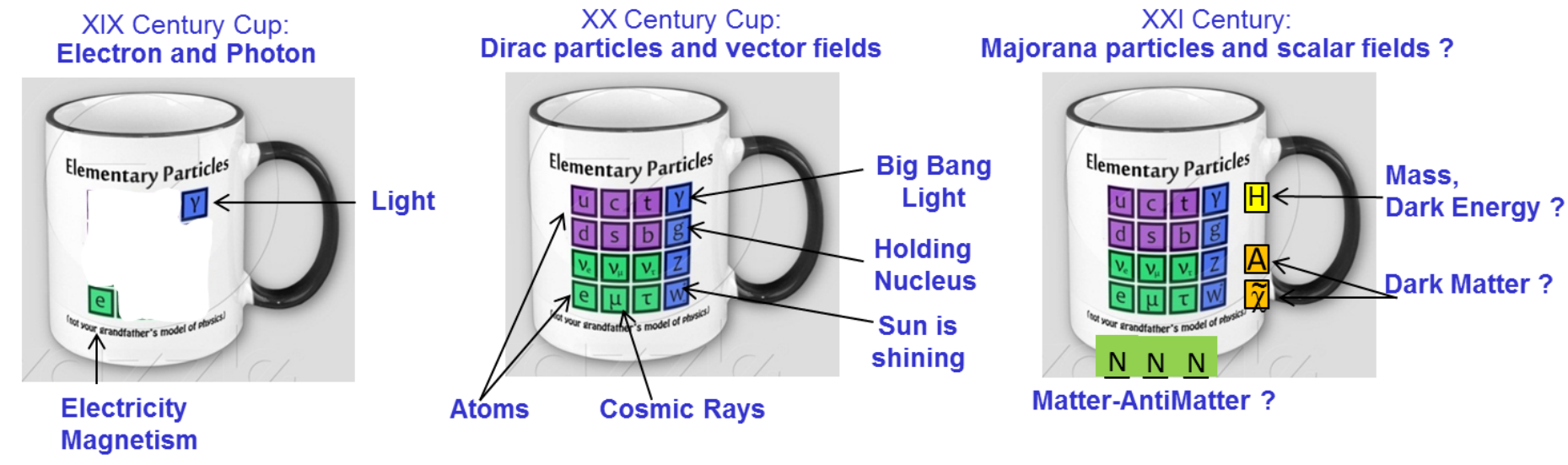

Fig. 47 Past, present and a possible future of particle physics.

Aside of these projects, new types of colliders are being developed, based on electron plasma activated by a laser. The main advantage of this completely new technology for particle physics is to increase the current accelerating gradient by a factor 1000 with respect to conventional Radio Frequency technology. Reaching 100 $\mathrm{GV} / \mathrm{m}$ will considerably reduce the collider size [172]. If this technology continues to follow the Livingstone law (Fig. 8) a 1-km TeV collider could be envisaged by 2035 and be competitive with CLIC, TLEP and VLHC projects.

\section{Conclusions}

Particle physics and cosmology are facing a particularly intriguing moment. Theoretically both are described by Standard Models with few parameters (triumph for the principle of simplicity?) and extremely robust against more and more precise experimental data. In particle physics, it's been 40 years without BSM discovery, despite the huge number of models predicting new physics close to the EW scale now extensively probed by LHC. In cosmology, $\Lambda$ CDM is still a good fit despite the reduction of allowed parameter space volume by $10^{5}$ during the last 15 years. Experimental findings are even more tantalizing: the cosmological constant is very small but not 0 (1998), the SM Higgs seems to exist at a mass of $125 \mathrm{GeV}$ and is apparently fine-tuned (2013). Both are pointing away from naturalness, even if the latter is still fresh and needs the full LHC program to be really conclusive.

Despite this quite unique situation for physics, the future should be paved by the understanding of current puzzles, i.e the nature of dark energy, dark matter and matter-antimatter asymmetry. Figure 47left/center shows that the discovery of new particles has always been a way to answer fundamental questions. So may be the $\mathrm{XXI}^{\text {rst }}$ century will continue the tradition as suggested by the right mug ... and ultimately decide whether our universe is natural or not?

Acknowledgments I'd like to express my gratitude to the organizers of the $100^{\text {th }}$ Les Houches Summer School for giving me the opportunity to deliver this lecture. It was also a real pleasure for me to stay some days in Les Houches and profit from the stimulating audience of cosmologists in a beautiful landscape.

\section{References}

1. S. L. Glashow and H. Georgi. New York Times Magazine (26-09-1982), 1982.

2. W. Buchmüller, Baryogenesis, Dark Matter and the Maximal Temperature of the Early Universe, Act. Phys. Polon. B43 (2012) 2153, arXiv:1212.3554

3. K. Schmitz, The B-L Phase Transition: Implications for Cosmology and Neutrinos. PhD thesis, 2012. arXiv:1307.3887. DESY-THESIS-2012-039.

4. N. Bohr, On the Constitution of Atoms and Molecules Part I, Phil. Mag. 26 (1913) 1.

5. N. Bohr, On the Constitution of Atoms and Molecules Part II, Phil. Mag. 26 (1913) 476.

6. S. L. Glashow, Partial symmetries of weak interactions, Nucl. Phys. 22 (1961) 579.

7. S. Weinberg, A model of leptons, Phys. Rev. Lett. 19 (1967) 1264.

8. A. Salam, Weak and electromagnetic interactions, in Elementary particle theory: relativistic group and analyticity. Almqvist/Wiksell. Proceedings of the $8^{\text {th }}$ Nobel symposium, 1968.

9. R. Brout and F. Englert, Broken symmetry and the mass of gauge vector mesons, Phys. Rev. Lett. 13 (1964) 321.

10. P. W. Higgs, Broken symmetries, massless particles and gauge fields, Phys. Lett. 12 (1964) 132. 
11. P. W. Higgs, Broken symmetries and the masses of gauge bosons, Phys. Rev. Lett. 13 (1964) 508.

12. G. S. Guralnik et al., Global conservation laws and massless particles, Phys. Rev. Lett. 13 (1964) 585.

13. P. W. Higgs, Spontaneous symmetry breakdown without massless bosons, Phys. Rev. 145 (1966) 1156.

14. T. W. B. Kibble, Symmetry breaking in non-Abelian gauge theories, Phys. Rev. 155 (1967) 1554.

15. G. 't Hooft and M. J. G. Veltman, Regularization and Renormalization of Gauge Fields, Nucl. Phys. B44 (1972) 189.

16. M. Gell-Mann, A schematic Model of Baryons and Mesons, Phys. Lett. 8 (1964) 214.

17. H. D. Politzer, Reliable Perturbative Results for Strong Interactions ?, Phys. Rev. Lett. 30 (1973) 1346.

18. D. J. Gross and F. Wilczek, Asymptotically Free Gauge Theories. I, Phys. Rev. D8 (1973) 3633.

19. D. J. Gross and F. Wilczek, Ultraviolet Behavior of Non-Abelian Gauge Theories, Phys. Rev. Lett. 30 (1973) 1343.

20. M. Kobayashi and T. Maskawa, CP-Violation in the Renormalizable Theory of Weak Interaction, Prog. Theo. Phys. 49 (1973) 652.

21. Gargamelle Coll., Observation of neutrino-like interactions without muon or electron in the Gargamelle neutrino experiment, Phys. Lett. B46 (1973) 138.

22. Gargamelle Coll., Search for elastic muon-neutrino electron scattering, Phys. Lett. B46 (1973) 121.

23. M. L. Perl et al., Evidence for Anomalous Lepton Production in $e^{+} e^{-}$Annihilation, Phys. Rev. Lett. 35 (1975) 1489.

24. DONUT Coll., Observation of tau neutrino interactions, Phys. Lett. B504 (2001) 218.

25. J.-E. Augustin et al., Discovery of a Narrow Resonance in $e^{+} e^{-}$Annihilation, Phys. Rev. Lett. 33 (1974) 1406.

26. J. J. Aubert et al., Experimental Observation of a Heavy Particle J, Phys. Rev. Lett. 33 (1974) 1404.

27. S. W. Herb et al., Observation of a Dimuon Resonance at $9.5 \mathrm{GeV}$ in 400-GeV Proton-Nucleus Collisions, Phys. Rev. Lett. 39 (1977) 252.

28. CDF Coll., Observation of Top Quark Production in Antipp Collisions, Phys. Rev. Lett. 74 (1995) 2626, arXiv: hep-ex/9503002

29. D0 Coll., Observation of the Top quark, Phys. Rev. Lett. 74 (1995) 2632, arXiv: hep-ex/9503003

30. TASSO Coll., Evidence for Planar Events in $e^{+} e^{-}$ Annihilation at High Energies, Phys. Lett. B86 (1979) 243.

31. PLUTO Coll., Evidence for Gluon Bremsstrahlung in $e^{+} e^{-}$Annihilations at High Energies, Phys. Lett. B86 (1979) 418.

32. MARK-J Coll., Discovery of Three-Jet Events and a Test of Quantum Chromodynamics at PETRA, Phys. Rev. Lett. 43 (1979) 830.

33. JADE Coll., Observation of planar three-jet events in $e+e$ - annihilation and evidence for gluon bremsstrahlung, Phys. Lett. B91 (1980) 142.

34. UA1 Coll., Experimental observation of isolated large transverse energy electrons with associated missing energy at $\sqrt{s}=540 \mathrm{GeV}$, Phys. Lett. B122 (1983) 103.

35. UA2 Coll., Observation of single isolated electrons of high transverse momentum in events with missing transverse energy at the CERN View the $\bar{p} p$ collider, Phys. Lett. B122 (1983) 476.
36. UA1 Coll., Experimental observation of lepton pairs of invariant mass around $95 \mathrm{GeV} / \mathrm{c} 2$ at the CERN SPS collider, Phys. Lett. B126 (1983) 398.

37. UA2 Coll., Evidence for $Z^{0} \rightarrow e^{+} e^{-}$at the CERN $\bar{p} p$ Collider, Phys. Lett. B129 (1983) 130.

38. ATLAS Coll., Observation of a new particle in the search for the Standard Model Higgs boson at the LHC, Phys. Lett. B716 (2012) 1, arXiv:1207.7214

39. CMS Coll., Observation of a new boson at a mass of $125 \mathrm{GeV}$ at the LHC, Phys. Lett. B716 (2012) 30, arXiv:1207.7235

40. G. Altarelli, Collider Physics within the Standard Model: a Primer, arXiv:1303.2842, 2013.

41. T. D. Lee and C.-N. Yang, Question of Parity Conservation in Weak Interactions, Phys. Rev. 104 (1956) 254.

42. H. Murayama, Supersymmetry Phenomenology. World Scientific, Singapore, 2000. arXiv: hep-ph/0002232.

43. L. Wolfenstein, Parametrization of the Kobayashi-Maskawa Matrix, Phys. Rev. Lett. 51 (1983) 1945.

44. S. Schael et al., Precision electroweak measurements on the $Z$ resonance, Phys. Rept. 427 (2006) 257, arXiv:hep-ex/0509008

45. M. Baak et al., The Electroweak Fit of the Standard Model after the Discovery of a New Boson at the LHC, Eur. Phys. J. C72 (2012) 2205, arXiv:1209.2716

46. C. M. G. Lattes et al., Processes Involving Charged Mesons, Nature 159 (1947) 694.

47. J. Burfening et al., Positive Mesons Produced by The 184-inch Berkeley Cyclotron, Phys. Rev. 75 (1949) 382 .

48. L. Evans and P. Briant, LHC machine, JINST 3 (2008) S08001.

49. M. Tigner, Does accelerator based particle physics have a future?, Phys. Today 54N1 (2001) 36.

50. ATLAS Coll., The ATLAS Experiment at the CERN Large Hadron Collider, JINST 3 (2008) S08003.

51. CMS Coll., The CMS Experiment at the CERN Large Hadron Collider, JINST 3 (2008) S08004.

52. G. F. Giudice, Big Science and the Large Hadron Collider, Phys. Perspect. 14 (2012) 95, arXiv: 1106.2443.

53. D. Froidevaux and P. Sphicas, General-Purpose detectors for the Large Hadron Collider, Ann. Rev. Nucl. Part. Sci. 56 (2006) 375.

54. S. Dittmaier et al., Handbook of LHC Higgs Cross Sections: 1. Inclusive Observables, arXiv:1101.0593, 2011.

55. S. Dittmaier et al., Handbook of LHC Higgs Cross Sections: 2. Differential Distributions, arXiv:1201.3084, 2012.

56. J. Ellis et al., A phenomenological profile of the Higgs boson, Nucl. Phys. B106 (1976) 292.

57. ATLAS Coll., Measurement of the Higgs boson mass from the $H \rightarrow \gamma \gamma$ and $H \rightarrow Z Z^{*} \rightarrow 4 l$ channels using $25 \mathrm{fb}^{-1}$ of pp collision data, Phys. Rev. D90 (2014) 052004, arXiv: 1406.3827.

58. CMS Coll., Measurements of the properties of the new boson with a mass near $125 \mathrm{GeV}$, CMS-PAS-HIG-14-009, 2014.

59. L. D. Landau, On the angular momentum of a two-photon system, Dokl. Akad. Nauk Ser. Fiz. 60 (1948) 207.

60. C.-N. Yang, Selection Rules for the Dematerialization of a Particle Into Two Photons, Phys. Rev. 77 (1950) 242. 
61. ATLAS Coll., Evidence for the spin-0 nature of the Higgs boson, Phys. Lett. B77 (2013) 120, arXiv:1307.1432

62. A. David et al., LHC HXSWG interim recommendations to explore the coupling structure of a Higgs-like particle, arXiv:1209.0040, 2012.

63. CMS Coll., Search for a heavy Higgs boson in the $H \rightarrow Z Z \rightarrow 2 l 2 \nu$ channel in pp collisions at $\sqrt{s}=7$ and $8 \mathrm{TeV}$, CMS-PAS-HIG-13-014, 2013.

64. C. Quigg and R. Shrock, Gedanken Worlds without Higgs: QCD-Induced Electroweak Symmetry Breaking, Phys. Rev. D79 (2009) 096002, arXiv:0901.3958

65. V. Mukhanov, Physical Foundations of Cosmology. Camb. U. Press, 2005.

66. V. A. Kuzmin et al., On the Anomalous Electroweak Baryon Number Non conservation in the Early Universe, Phys. Lett. B155 (1985) 36.

67. M. E. Shaposhnikov, Possible Appearance of the Baryon Asymmetry of the Universe in an Electroweak Theory, JETP Lett. 44 (1986) 465.

68. M. E. Shaposhnikov, Baryon Asymmetry of the Universe in Standard Electroweak Theory, Nucl. Phys. B287 (1987) 757.

69. A. H. Guth, The Inflationary Universe: A Possible Solution to the Horizon and Flatness Problems, Phys. Rev. D23 (1981) 347.

70. A. D. Linde, A New Inflationary Universe Scenario: A Possible Solution of the Horizon, Flatness, Homogeneity, Isotropy and Primordial Monopole Problems, Phys. Lett. B108 (1982) 389.

71. A. Albrecht and P. J. Steinhardt, Cosmology for Grand Unified Theories with Radiatively Induced Symmetry Breaking, Phys. Rev. Lett. 48 (1982) 1220.

72. A. D. Linde, Chaotic Inflation, Phys. Lett. B129 (1983) 177

73. N. D. Birrell and P. C. W. Favies, Quantum Fields in Curved Space. Camb. U. Press, 2002.

74. A. De Simone et al., Running inflation in the Standard Model, Phys. Lett. B678 (2009) 1, arXiv:0812.4946.

75. F. Bezrukov et al., Standard Model Higgs boson mass from inflation, Phys. Lett. B675 (2009) 88, arXiv:0812.4950

76. F. Bezrukov and M. E. Shaposhnikov, Standard Model Higgs boson mass from inflation: two loop analysis, JHEP 0907 (2009) 089, arXiv:0904.1537

77. J. Martin et al., Encyclopedia Inflationaris, arXiv:1303.3787, 2013.

78. C. P. Burgess et al., Comment on Higgs Inflation and Naturalness, JHEP 1007 (2010) 007, arXiv: 1002.2730

79. S. Weinberg, The Cosmological Constant Problem, Rev. Mod. Phys. 61 (1989) 1.

80. J. Martin, Everything You Always Wanted To Know About The Cosmological Constant Problem (But Were Afraid To Ask), Comptes Rendus Physique 13 (2012) 566, arXiv: 1205.3365

81. C. P. Burgess, The cosmological constant problem, Proceedings of the $100^{t h}$ Les Houches Summer School, 2013. arXiv:1309.4133

82. J. Ellis et al., The Probable Fate of the Standard Model, Phys. Lett. B679 (2009) 369, arXiv:0906.0954

83. G. Degrassi et al., Higgs mass and vacuum stability in the Standard Model at NNLO, JHEP 1208 (2012) 098, arXiv:1205.6497

84. E. Gildener, Gauge Symmetry Hierarchies, Phys. Rev. D14 (1976) 1667.
85. S. Weinberg, Gauge Hierarchies, Phys. Lett. B82 (1979) 387.

86. G. F. Giudice, Naturally Speaking: The Naturalness Criterion and Physics at the LHC. World Scientific, Singapore, 2008. arXiv:0801.2562.

87. J. L. Feng, Dark Matter Candidates from Particle Physics and Methods of Detection, Ann. Rev. Astron. Astrophys. 48 (2010) 495, arXiv: 1003.0904.

88. D. E. Morrissey et al., Physics searches at the $L H C$, Phys. Rept. 515 (2012) 1, arXiv:0912.3259

89. S. P. Martin, A Supersymmetry Primer, arXiv:hep-ph/9709356, 2011.

90. S. Dimopoulos and H. Georgi, Softly Broken Supersymmetry and SU(5), Nucl. Phys. B193 (1981) 150.

91. L. Hall. Searches for SUSY at the LHC, LBL Workshop, 19-21 Oct 2011, 2011.

92. ATLAS Coll., Supersymmetry Public Results, http://twiki.cern.ch/twiki/bin/view/AtlasPublic/SupersymmetryPublicResults, 2013.

93. CMS Coll., Supersymmetry Public Results, https://twiki.cern.ch/twiki/bin/view/CMSPublic/PhysicsResultsSUS, 2013.

94. I. Melzer-Pellmann and P. Pralavorio, Lessons for SUSY from the LHC after the first run, Eur. Phys. J. C74 (2014) 2801, arXiv:1404.7191.

95. CMS Coll., Search for neutral MSSM Higgs bosons decaying to a pair of tau leptons in pp collisions, JHEP 1410 (2014) 160, arXiv:1408.3316.

96. CMS Coll., Search for a Higgs boson decaying into a $b$-quark pair and produced in association with $b$ quarks in $p p$ collisions at $\sqrt{s}=7 \mathrm{TeV}$, Phys. Lett. B722 (2013) 207, arXiv: 1302.2892.

97. ATLAS Coll., Search for charged Higgs bosons in the $\tau+$ jets final state with $p p$ collision data recorded at $\sqrt{s}=8 \mathrm{TeV}$, ATLAS-CONF-2013-090, 2013.

98. M. Carena et al., Suggestions for Improved Benchmark Scenarios for Higgs-Boson Searches at LEPQ, arXiv:hep-ph/9912223, 1999.

99. I. Hinchliffe et al., Precision SUSY measurements at CERN LHC, Phys. Rev. D55 (1997) 5520, arXiv: hep-ph/9610544

100. D. Tovey, Measuring the SUSY Mass Scale at the $L H C$, Phys. Lett. B498 (2001) 1, arXiv:hep-ph/0006276

101. ATLAS Coll., Search for squarks and gluinos in final states with jets and missing transverse momentum using $\sqrt{s}=8$ TeV $p p$ collision data, JHEP 1409 (2014) 176, arXiv:1405.7875.

102. ATLAS Coll., Search for new phenomena in final states with large jet multiplicities and missing transverse momentum at $\sqrt{s}=8$ TeV pp collisions, JHEP 1310 (2013) 130, arXiv: 1308.1841.

103. ATLAS Coll., Search for supersymmetry at $\sqrt{s}=8$ TeV in final states with jets and two same-sign leptons or three leptons, JHEP 1406 (2014) 035, arXiv: 1404.2500 .

104. ATLAS Coll., Search for strong production of supersymmetric particles in final states with missing transverse momentum and at least three b-jets at $\sqrt{s}$ $=8 \mathrm{TeV}$ pp collisions, JHEP 1410 (2014) 024, arXiv: 1407.0600 .

105. ATLAS Coll., Search for squarks and gluinos in events with isolated leptons, jets and missing transverse momentum at $\sqrt{s}=8 \mathrm{TeV}$, ATLAS-CONF-2013-062, 2013. 
106. ATLAS Coll., Search for diphoton events with large missing transverse momentum in $7 \mathrm{TeV}$ pp collision data, Phys. Lett. B718 (2012) 411, arXiv:1209.0753

107. D. Tovey, On measuring the masses of pair-produced semi-invisibly decaying particles at hadron colliders, JHEP 0804 (2008) 034, arXiv:0802.2879

108. G. Polesello and D. Tovey, Supersymmetric particle mass measurement with the boost-corrected contransverse mass, JHEP 1003 (2010) 030, arXiv:0910.0174

109. ATLAS Coll., Search for direct third-generation squark pair production in final states with missing transverse momentum and two b-jets in $\sqrt{s}=8$ TeV pp collisions, JHEP 1310 (2013) 189, arXiv:1308.2631

110. ATLAS Coll., Search for direct production of the top squark in the all-hadronic final state in pp collisions at $\sqrt{s}=8 \mathrm{TeV}$, JHEP 1409 (2014) 015, arXiv:1406.1122

111. ATLAS Coll., Search for top squark pair production in final states with one isolated lepton, jets, and missing transverse momentum in $\sqrt{s}=8$ TeV pp collisions, JHEP 1411 (2014) 118, arXiv:1407.0583

112. ATLAS Coll., Search for direct top-squark pair production in final states with two leptons in $p p$ collisions at $\sqrt{s}=8 \mathrm{TeV}$, JHEP 1406 (2014) 124, arXiv: 1403.4853

113. ATLAS Coll., Search for pair-produced third-generation squarks decaying via charm quarks or in compressed supersymmetric scenarios in pp collisions at $\sqrt{s}=8 \mathrm{TeV}$, Phys. Rev. D90 (2014) 052008, arXiv: 1408.0608.

114. ATLAS Coll., Search for direct production of charginos and neutralinos in events with three leptons and missing transverse momentum in $\sqrt{s}=8 \mathrm{TeV} p p$ collisions, JHEP 1404 (2014) 169, arXiv:1402.7029

115. ATLAS Coll., Search for direct production of charginos, neutralinos and sleptons in final states with two leptons and missing transverse momentum in $p p$ collisions at $\sqrt{s}=8 \mathrm{TeV}$, JHEP 1405 (2014) 071, arXiv: 1403.5294

116. M. W. Cahill-Rowley et al., The New Look pMSSM with Neutralino and Gravitino LSPs, Eur. Phys. J. C72 (2012) 2156, arXiv:1206.4321

117. M. W. Cahill-Rowley et al., The Higgs Sector and Fine-Tuning in the pMSSM, Phys. Rev. D86 (2012) 075015, arXiv: 1206.5800

118. R. Barbier et al., R-parity violating supersymmetry, Phys. Rept. 420 (2005) 1, arXiv:hep-ph/0406039.

119. U. Ellwanger et al., The Next-to-Minimal Supersymmetric Standard Model, Phys. Rept. 496 (2010) 1, arXiv:0910.1785.

120. A. Arvanitaki et al., Mini-Split, JHEP 1302 (2013) 126, arXiv:1210.0555

121. ATLAS Coll., Search for charginos nearly mass-degenerate with the lightest neutralino based on a disappearing-track signature in pp collisions at $\sqrt{s}=8$ TeV, Phys. Rev. D88 (2013) 112006, arXiv: 1310.3675

122. ATLAS Coll., Search for long-lived stopped R-hadrons decaying out-of-time with pp collisions, Phys. Rev. D88 (2013) 112003, arXiv: 1310.6584

123. ATLAS Coll., Search for supersymmetry in events with four or more leptons at $\sqrt{s}=8 \mathrm{TeV}$ pp collisions, Phys. Rev. D90 (2014) 052001, arXiv: 1405.5086

124. ATLAS Coll., Search for massive particles in multijet signatures in $\sqrt{s}=8 \mathrm{TeV}$ pp collisions at the LHC, ATLAS-CONF-2013-091, 2013.
125. N. Arkani-Hamed et al., The hierarchy problem and new dimensions at a millimeter, Phys. Lett. B429 (1998) 263, arXiv:hep-ph/9803315

126. G. F. Giudice et al., Quantum gravity and extra dimensions at high-energy colliders, Nucl. Phys. B544 (1999) 3, arXiv:hep-ph/9811291.

127. CMS Coll., Search for new physics in monojet events in pp collisions at $\sqrt{s}=8 \mathrm{TeV}$, Submitted to Eur. Phys. J. C, arXiv:1408.3583, 2014.

128. ATLAS Coll., Search for Extra Dimensions in diphoton events using pp collisions recorded at $\sqrt{s}=7$ TeV at the LHC, New J. Phys. 15 (2013) 043007, arXiv: 1210.8389.

129. CMS Coll., Search for microscopic black holes in $p p$ collisions at $\sqrt{s}=8 \mathrm{TeV}$, JHEP 1307 (2013) 178, arXiv: 1303.5338.

130. ATLAS Coll., Search for microscopic black holes in a like-sign dimuon final state using large track multiplicity, Phys. Rev. D88 (2013) 072001, arXiv: 1308.4075.

131. ATLAS Coll., Search for Quantum Black-Hole Production in High-Invariant-Mass Lepton+Jet Final States Using pp Collisions at $\sqrt{s}=8$ TeV, Phys. Rev. Lett. 112 (2014) 091804, arXiv:1311.2006

132. L. Randall and R. Sundrum, A Large Mass Hierarchy from a Small Extra Dimension, Phys. Rev. Lett. 83 (1999) 3370, arXiv:hep-ph/9905221

133. ATLAS Coll., Search for high-mass dilepton resonances in $p p$ collisions at $\sqrt{s}=8 \mathrm{TeV}$, Phys. Rev. D90 (2014) 052005, arXiv:1405.4123.

134. H. Davoudiasl et al., Bulk Gauge Fields in the Randall-Sundrum Model, Phys. Lett. B473 (2000) 43, arXiv: hep-ph/9911262

135. J. Hewett and M. Spiropulu, Particle physics probes of extra space-time dimensions, Ann. Rev. Nucl. Part. Sci. 52 (2002) 397, arXiv: hep-ph/0205106

136. CMS Coll., Search for Anomalous Top Quark Pair Production in the Boosted All-Hadronic Final State using pp Collisions at $\sqrt{s}=8 \mathrm{TeV}$, CMS-PAS-B2G-12-005, 2013.

137. CMS Coll., Searches for new physics using the ttbar invariant mass distribution in pp collisions at $\sqrt{s}=8$ TeV, Phys. Rev. Lett. 111 (2013) 211804, arXiv: 1309.2030

138. CMS Coll., Search for massive resonances in dijet systems containing jets tagged as $W$ or $Z$ boson decays in $p p$ collisions at $\sqrt{s}=8 \mathrm{TeV}$, JHEP 1408 (2014) 173, arXiv: 1405.1994 .

139. CMS Coll., Search for massive resonances decaying into pairs of boosted bosons in semi-leptonic final states at $\sqrt{s}=8 \mathrm{TeV}$, JHEP 1408 (2014) 174, arXiv: 1405.3447.

140. G. F. Giudice et al., The Strongly-Interacting Light Higgs, JHEP 0706 (2007) 045, arXiv: hep-ph/0703164

141. CMS Coll., Inclusive search for a vector-like T quark with charge $2 / 3$ in pp collisions at $\sqrt{s}=8 \mathrm{TeV}$, Phys. Lett. B729 (2014) 149, arXiv:1311.7667

142. CMS Coll., Search for top-quark partners with charge $5 / 3$ in the same-sign dilepton final state, Phys. Rev. Lett. 112 (2014) 171801, arXiv:1312.2391

143. G. Cacciapaglia et al., Higgs couplings beyond the Standard Model, JHEP 1303 (2013) 029, arXiv: 1210.8120 .

144. ATLAS Coll., Search for New Phenomena in the Dijet Mass Distribution updated using pp Collisions at $\sqrt{s}=8 T e V$, Submitted to Phys. Rev. D, arXiv:1407.1376, 2014. 
145. ATLAS Coll., Search for new phenomena in dijet mass and angular distributions using pp collisions at $\sqrt{s}=7$ TeV, JHEP 1301 (2013) 029, arXiv:1210.1718.

146. CMS Coll., Exotica Public Results, https://twiki.cern.ch/twiki/bin/view/CMSPublic/PhysicsResultsEXO, 2013.

147. ATLAS Coll., Exotics Public Results, http://twiki.cern.ch/twiki/bin/view/AtlasPublic/ExoticsPublicResults, 2013.

148. J. L. Hewett et al., Fundamental Physics at the Intensity Frontier, arXiv:1205.2671, 2012.

149. V. Cirigliano and M. J. Ramsey-Musolf, Low energy probes of physics beyond the standard model, Prog. Part. Nucl. Phys. 71 (2013) 2, arXiv: 1304.0017.

150. E.-K. Park, Contribution to DMSAG report, July 18, 2007, http://science.energy.gov/hep/hepap/reports/, 2007.

151. J. Silk, Dark Matter direct and indirect detection, Proceedings of the $100^{t h}$ Les Houches Summer School, 2013.

152. G. Bertone et al., Particle Dark Matter: Evidence, Candidates and Constraints, Phys. Rept. 405 (2005) 279, arXiv:hep-ph/0404175

153. Y. Bai et al., The Tevatron at the frontier of dark matter direct detection, JHEP 1012 (2010) 048, arXiv:1005.3797

154. J. Goodman et al., Constraints on Light Majorana Dark Matter from Colliders, Phys. Lett. B695 (2011) 185, arXiv:1005.1286

155. M. W. Cahill-Rowley et al., Complementarity and Searches for Dark Matter in the pMSSM, arXiv:1305.6921, 2013.

156. A. Djouadi et al., Direct Detection of Higgs-Portal Dark Matter at the LHC, Eur. Phys. J. C73 (2013) 2455, arXiv: 1205.3169 .

157. Super-Kamiokande Coll., Evidence for oscillation of atmospheric neutrinos, Phys. Rev. Lett. 81 (1998) 1562, arXiv: hep-ex/9807003

158. Daya Bay Coll., Observation of electron-antineutrino disappearance at Daya Bay, Phys. Rev. Lett. 108 (2012) 171803, arXiv:1203.1669

159. Troitsk Coll., An upper limit on electron antineutrino mass from Troitsk experiment, Phys. Rev. D84 (2011) 112003, arXiv:1108.5034

160. P. Minkowski, $\mu \rightarrow e \gamma$ at a rate at one out of billion muon decays, Phys. Lett. B67 (1977) 421.

161. M. Gell-Mann et al., Complex spinors and unified theories, Conf. Proc. C790927 (1979) 315, arXiv: 1306.4669

162. T. Yanagida, Horizontal gauge symmetry and masses of neutrinos, Sawada/Sugamoto, Proceedings of the Workshop on Unified Theories and Baryon Number in the Universe, 1979.

163. R. N. Mohapatra and G. Senjanovic, Neutrino Mass and Spontaneous Parity Violation, Phys. Rev. Lett. 44 (1980) 912

164. M. Yoshimura, Unified Gauge Theories and the Baryon Number of the Universe, Phys. Rev. Lett. 41 (1978) 281.

165. M. Fukugita and T. Yanagida, Baryogenesis Without Grand Unification, Phys. Lett. B174 (1986) 45.

166. S. Blanchet and P. Di Bari, The minimal scenario of leptogenesis, New J. Phys. 14 (2012) 125012, arXiv: 1211.0512

167. A. D. Sakharov, Violation of $C P$ invariance, $C$ asymmetry, and baryon asymmetry of the universe, JETP Lett. 5 (1967) 24.
168. S. Barr et al., Magnitude of the cosmological baryon asymmetry, Phys. Rev. D20 (1979) 2494.

169. M. E. Shaposhnikov, Is there a new physics between electroweak and Planck scales?, arXiv:0708.3550, 2007.

170. R. Aleksan et al., Physics Briefing Book: Input for the Particle Physics European Strategy Group, CERN-ESG-005, 2013.

171. M. E. Peskin, Comparison of $L H C$ and $I L C$ Capabilities for Higgs Boson Coupling Measurements, arXiv:1207.2516, 2013.

172. W. Leemans and E. Esarey, Laser-driven plasma-wave electron accelerators, Phys. Today 62N3 (2009) 44. 